NASA/TM-2002-211712

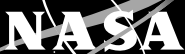

Effects of Fuel Distribution on Detonation Tube Performance

Hugh Douglas Perkins

Glenn Research Center, Cleveland, Ohio 
Since its founding, NASA has been dedicated to the advancement of aeronautics and space science. The NASA Scientific and Technical Information (STI) Program Office plays a key part in helping NASA maintain this important role.

The NASA STI Program Office is operated by Langley Research Center, the Lead Center for NASA's scientific and technical information. The NASA STI Program Office provides access to the NASA STI Database, the largest collection of aeronautical and space science STI in the world. The Program Office is also NASA's institutional mechanism for disseminating the results of its research and development activities. These results are published by NASA in the NASA STI Report Series, which includes the following report types:

- $\quad$ TECHNICAL PUBLICATION. Reports of completed research or a major significant phase of research that present the results of NASA programs and include extensive data or theoretical analysis. Includes compilations of significant scientific and technical data and information deemed to be of continuing reference value. NASA's counterpart of peerreviewed formal professional papers but has less stringent limitations on manuscript length and extent of graphic presentations.

- TECHNICAL MEMORANDUM. Scientific and technical findings that are preliminary or of specialized interest, e.g., quick release reports, working papers, and bibliographies that contain minimal annotation. Does not contain extensive analysis.

- CONTRACTOR REPORT. Scientific and technical findings by NASA-sponsored contractors and grantees.
- CONFERENCE PUBLICATION. Collected papers from scientific and technical conferences, symposia, seminars, or other meetings sponsored or cosponsored by NASA.

- SPECIAL PUBLICATION. Scientific, technical, or historical information from NASA programs, projects, and missions, often concerned with subjects having substantial public interest.

- TECHNICAL TRANSLATION. Englishlanguage translations of foreign scientific and technical material pertinent to NASA's mission.

Specialized services that complement the STI Program Office's diverse offerings include creating custom thesauri, building customized data bases, organizing and publishing research results ... even providing videos.

For more information about the NASA STI Program Office, see the following:

- Access the NASA STI Program Home Page at http://www.sti.nasa.gov

- E-mail your question via the Internet to help@sti.nasa.gov

- Fax your question to the NASA Access Help Desk at 301-621-0134

- Telephone the NASA Access Help Desk at 301-621-0390

- Write to:

NASA Access Help Desk

NASA Center for AeroSpace Information 7121 Standard Drive

Hanover, MD 21076 
NASA/TM-2002-211712

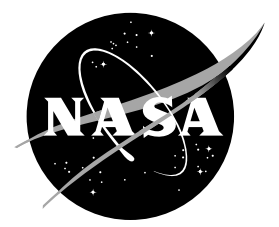

\section{Effects of Fuel Distribution on Detonation Tube Performance}

Hugh Douglas Perkins

Glenn Research Center, Cleveland, Ohio

National Aeronautics and

Space Administration

Glenn Research Center 


\section{Acknowledgments}

I would like to acknowledge the patience and support of my thesis advisor Dr. C.J. Sung of Case Western Reserve University in guiding this work. I would also like to acknowledge the NASA Glenn Research Center for its support of my program of study and the development of this paper.

The Aerospace Propulsion and Power Program at NASA Glenn Research Center sponsored this work.

Available from

NASA Center for Aerospace Information 7121 Standard Drive

Hanover, MD 21076
National Technical Information Service 5285 Port Royal Road Springfield, VA 22100 


\title{
Effects of Fuel Distribution on Detonation Tube Performance
}

\author{
Hugh Douglas Perkins \\ National Aeronautics and Space Administration \\ Glenn Research Center \\ Cleveland, Ohio 44135
}

\begin{abstract}
Summary
A pulse detonation engine (PDE) uses a series of high frequency intermittent detonation tubes to generate thrust. The process of filling the detonation tube with fuel and air for each cycle may yield non-uniform mixtures. Lack of mixture uniformity is commonly ignored when calculating detonation tube thrust performance. In this study, detonation cycles featuring idealized non-uniform $\mathrm{H}_{2} /$ air mixtures were analyzed using the SPARK two-dimensional Navier-Stokes CFD code with a 7-step $\mathrm{H}_{2} /$ air reaction mechanism. Mixture non-uniformities examined included axial equivalence ratio gradients, transverse equivalence ratio gradients, and partially fueled tubes. Three different average test section equivalence ratios $(\Phi)$, stoichiometric $(\Phi=1.00)$, fuel lean $(\Phi=0.90)$, and fuel rich $(\Phi=1.10)$, were studied. All mixtures were detonable throughout the detonation tube. It was found that various mixtures representing the same test section equivalence ratio had specific impulses within $1 \%$ of each other, indicating that good fuel/air mixing is not a prerequisite for optimal detonation tube performance.
\end{abstract}

Nomenclature

$b_{i} \quad$ Fluid element body forces, $i^{\text {th }}$ dimension

c Speed of sound

$\mathrm{d}_{\mathrm{i}} \quad$ Dimensional characteristic boundary derivatives

$f_{i} \quad$ Mass fraction of the $i^{\text {th }}$ species

$h_{i} \quad$ Enthalpy of the $i^{\text {th }}$ species

$i_{\text {ref }} \quad$ Centerpoint of X-grid spline

k Thermal conductivity

$\mathrm{k}_{\mathrm{b}} \quad$ Backward reaction rate

$\mathrm{k}_{\mathrm{f}} \quad$ Forward reaction rate

$\mathrm{n} \quad$ Arrhenius expression temperature exponent

ns Number of chemical species

$\mathrm{p} \quad$ Static pressure

$\mathrm{p}_{\mathrm{L}} \quad$ Local tube exit pressure

$\mathrm{p}_{\mathrm{A}} \quad$ Ambient (atmospheric) pressure

$\vec{q} \quad$ Fluid element heat flux

$\mathrm{t}$ Time 


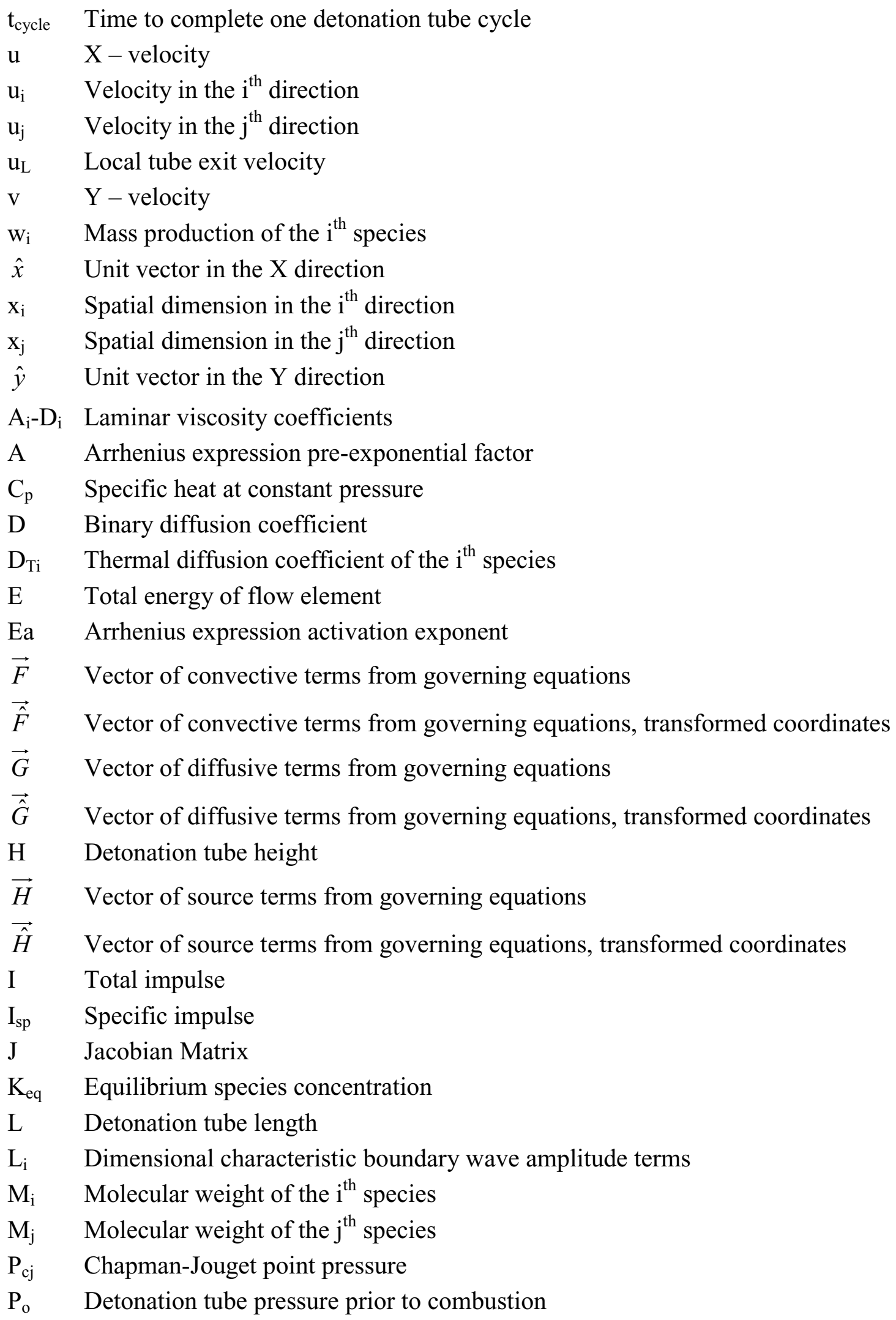




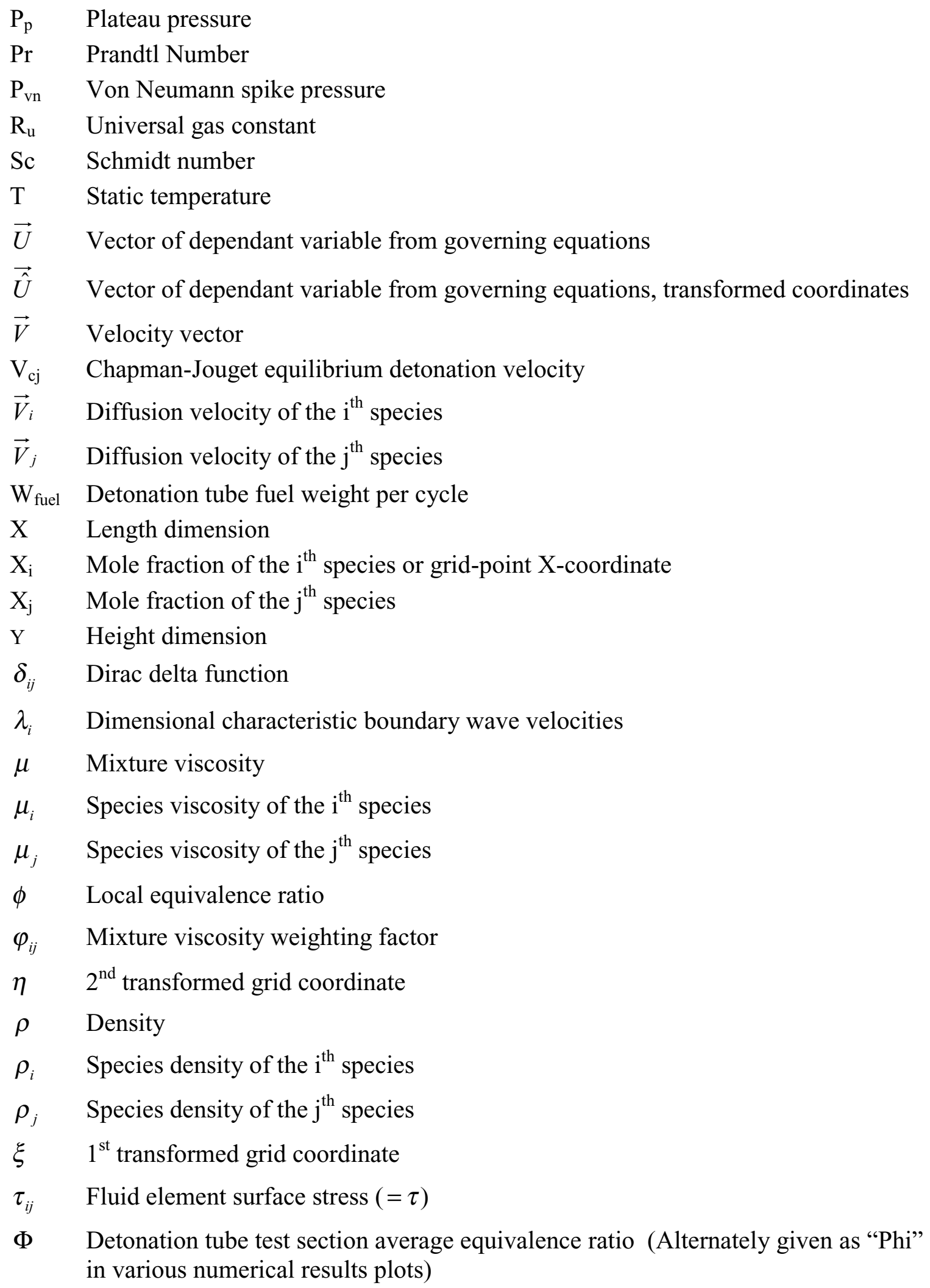




\subsection{Introduction}

\subsection{Motivation of Study}

The concept of a pulse detonation engine (PDE) for aerospace propulsion system applications is not new. The work at the University of Michigan in the 1950's (Nichols et al., 1957) is a prime example. However, it was not until repetitive detonations with gaseous hydrocarbon fuels at frequencies at or above 25 detonations per second were demonstrated at the Naval Post-Graduate School in the mid-1980's that it became apparent that practical devices might be possible, as described by Coleman (2001). These advances and others over the past decade have stimulated interest in the mainstream propulsion development community (Kailasanath, 1999; Kaemming, 2001). Both government and industry entities are currently considering the application of PDE's and derivative configurations to missions as diverse as Single-Stage-To-Orbit (SSTO) launch vehicles, rocket upper stages, commercial aircraft, missiles, and tactical aircraft (Coleman, 2001). At the heart of each of these unique propulsion systems is a series of high frequency intermittent (pulse) detonation tubes.

PDE's are attractive for several reasons. First, Kailasanath and Patniak (1999), among others, have shown that the thermal efficiency of the detonation cycle can approach two times the thermal efficiency of the standard Brayton cycle found in gas turbine engines, $49 \%$ versus $27 \%$ in an example case where both combustion processes began at the same initial pressure. Second, since static pressure increases substantially during detonative combustion, instead of slightly decreasing as in deflagrative combustion, it is possible to decrease the amount of compression required before the combustion process, thus leading to simplified, lighter, lower cost engine architectures. For some missions, such as air-launched missiles, it is possible to eliminate all mechanical pre-compression and operate the PDE as a "supercharged" ramjet, even at relatively low subsonic Mach numbers. Lastly, a PDE detonation chamber does not have to be round, nor do the detonation chambers have to be grouped in circular arrays if turbomachinery components have been eliminated, thus leading to a more aerodynamically efficient airframe integration than with a gas turbine engine. These PDE advantages will become more apparent in the following sections as the detonation tube architecture and engine gas dynamics are explored.

\subsection{PDE Architecture Description}

A variety of architectures have been proposed for pulse detonation engines, differing materially in tube geometry, air valve design, fuel injector placement, and other operational features. Figure 1 shows a generic configuration for an airbreathing pulse detonation tube. To begin a new cycle, the air valves on the left are opened to admit air from the inlet plenum. Fuel is injected into the incoming air stream as it enters the 
initiator tube and the main detonation tube. Typically, the start of fuel injection is delayed slightly after the air valves are opened in order to allow a thin buffer of unfueled air to form between the exhaust products from the previous cycle and the fresh charge, preventing pre-ignition. After the inlet fuel and air valves are closed, combustion is initiated using the spark plug in the initiator tube. The ensuing deflagration quickly accelerates into a detonation, aided by the small diameter of the initiator tube and typically by some amount of oxygen enrichment in the initiator tube.

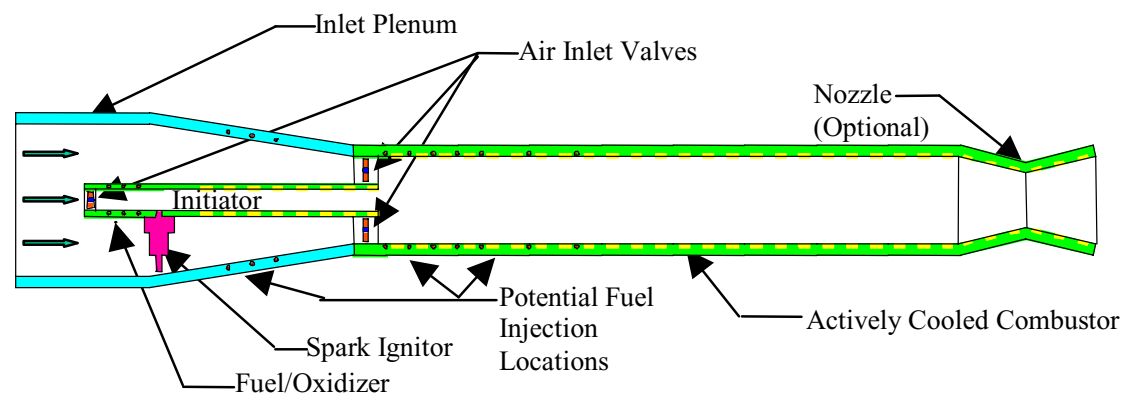

Figure 1 - Basic Pulse Detonation Combustor Tube Architecture

Once formed, the detonation wave travels down the initiator tube at a characteristic detonation wave speed in excess of $1500 \mathrm{~m} / \mathrm{s}$, depending on the fuel and oxidizer combination used and the initial conditions (pressure, temperature, stoichiometry). The detonation wave then propagates out of the initiator tube, detonating the main charge. Care must be taken in selecting appropriate geometric parameters (area ratio, rate of area change, cross section) that will allow the transition of the detonation wave into the larger tube. In some cases, the detonation may initially fail (shock/combustion decoupling) upon exiting the initiator tube, but will then re-initiate from hot spots in the main tube caused by intersecting shocks.

Two additional features of the detonation tube shown in Figure 1 that aid in the initiation and propagation of the detonation wave are the convergent-divergent (C-D) nozzle and the actively cooled combustor walls. The nozzle, when employed, is used to provide a higher initial (pre-detonation) pressure in the tube by restricting the outflow from the tube during the fill process. This higher initial pressure aids in the initiation of a detonation, since detonability is a function of both pressure and temperature (Kuo, 1986). A higher initial pressure also increases thrust potential due to the corresponding increase in peak and plateau pressures caused by the pressure multiplying effect of the detonation wave. In the common configuration, the detonation cycle is also enabled by the presence of the actively cooled combustor walls. The wall temperature must be maintained below the autoignition temperature of the fuel/air mixture being used to prevent deflagrative 
mode ignition prior to the initiation of the detonation. However, any mixing or buffering scheme that keeps the flammable mixture away from the wall could also be used to prevent autoignition.

\subsection{Detonation Wave and PDE Wave Cycle Descriptions}

Once fully initiated, the detonation wave assumes the characteristic Zeldovich, Von Neumann, Doring (ZND) structure, shown in the fixed, or laboratory, coordinates in Figure 2, taken in part from Kuo (1986). The leading shock compresses the fuel/oxidizer mixture (A) to a pressure and temperature sufficient for rapid combustion (B). This point is known as the Von Neumann spike, and is approximately equal to the pressure and temperature rise observed across a frozen shock moving at the same Mach number as the detonation wave. After a short kinetic delay, combustion occurs with a characteristic decrease in static pressure. Point $(C)$ corresponds to the point at which combustion is complete, know as the Chapman-Jouget (CJ) point. The CJ point is uniquely defined by the pre-detonation conditions and represents the point at which the local Mach number in the combustion products equals 1 in a reference frame fixed to the detonation wave. While it is possible to have a detonation which does not end in the CJ point, such detonations are generally unstable, and so the leading shock will typically self-adjust its speed (and therefore strength) so as to create the CJ point at the end of the combustion zone. The resulting shock Mach number, referenced to the speed of sound in the unburned gas, is known as the CJ Mach number, and is again uniquely defined by the unburned conditions.

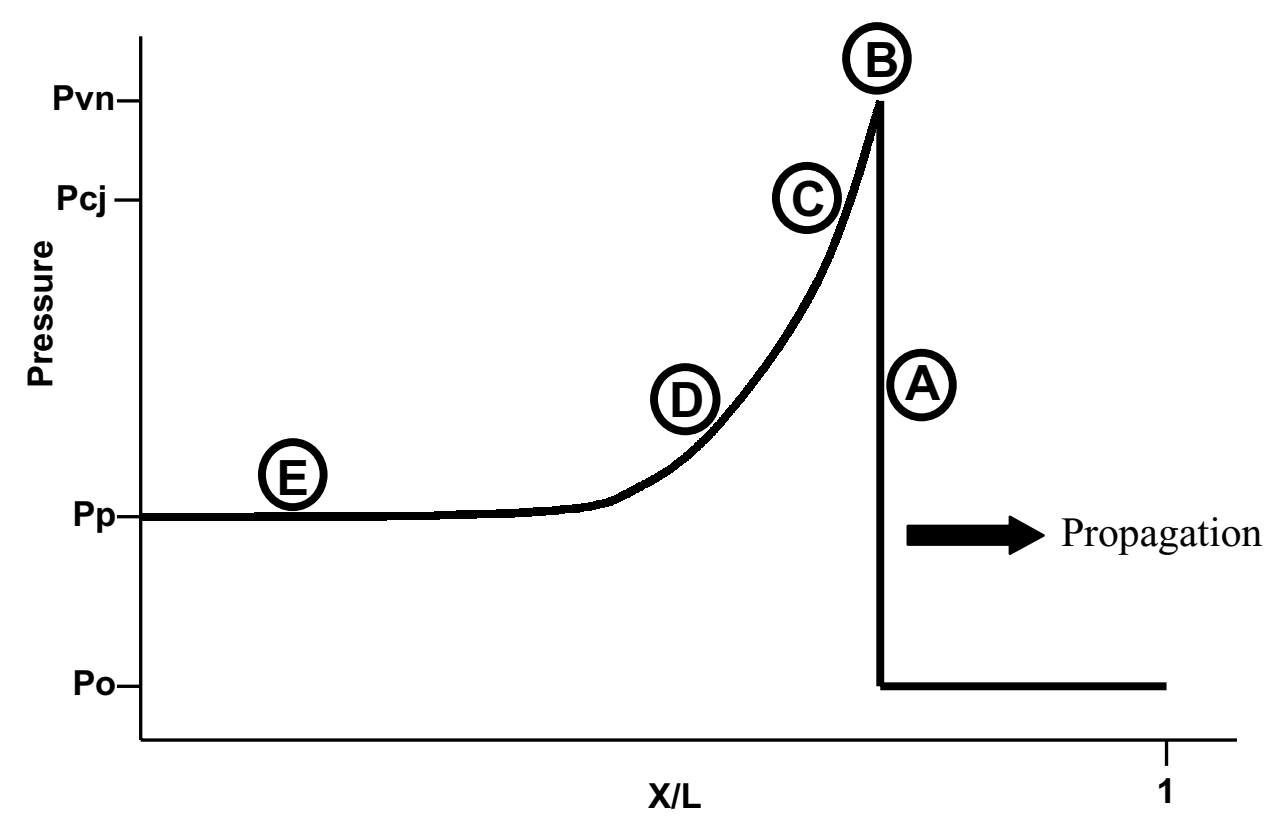

Figure $2-$ ZND Detonation Structure in a Tube (Laboratory Coordinates) 
In order to maintain continuity in the closed tube, a series of expansion waves (D) propagate toward the closed end of the detonation tube from the detonation wave front to reduce the pressure from the $\mathrm{CJ}$ pressure to what is commonly referred to as the plateau pressure (E), reconciling the high velocity flow following the detonation wave with the zero velocity requirement at the closed end wall. The tube then exhausts through the open end, with the blow down characteristics governed by the reflected expansion and compression waves propagating through the tube. Once the tube has reached a pressure sufficiently close to ambient pressure, the air inlet valves are opened to begin recharging the detonation tube leading to the initiation of a new cycle. The overall detonation cycle is shown graphically in Figure 3.

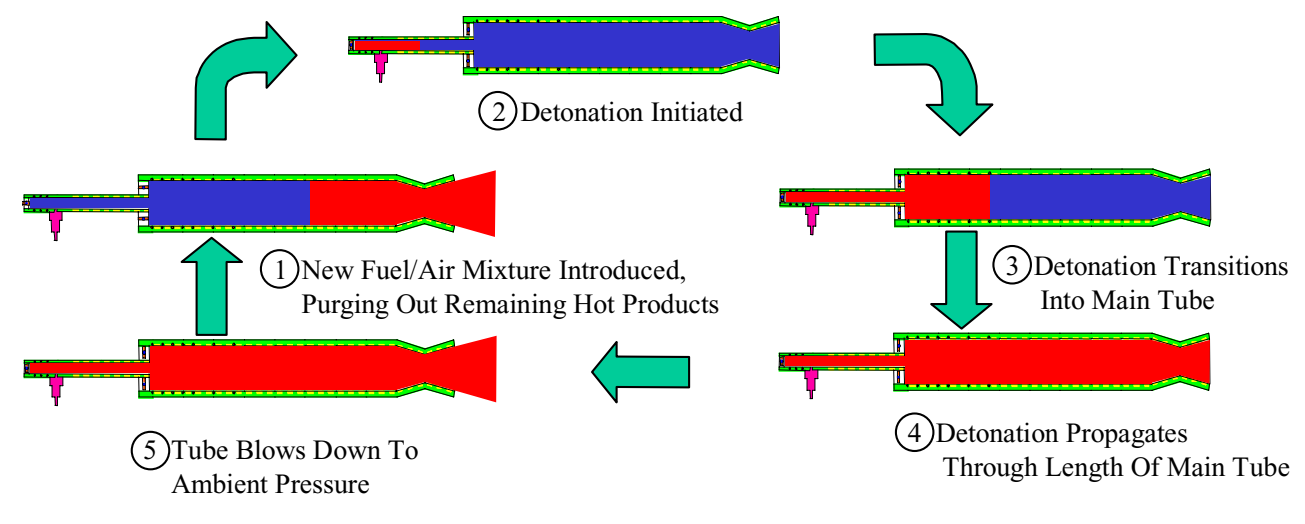

Figure 3 - Pulse Detonation Combustor Operational Cycle

\subsection{Scope of Numerical Study}

It is likely that in any application of detonative combustion for propulsion, the fuel and air will not be uniformly mixed, as is often assumed. Non-uniformity in fuel distribution may be created intentionally or may result from hardware limitations, and may be either axial or transverse, or both, depending on the source. Possible sources of non-uniform fuel distribution include, but are not limited to,

- Combustion products/fresh charge buffering (purge)

- Engine throttling

- Emissions control

- Wall heat transfer control

- Detonation wave shape/strength control

- Air and fuel valve transients

- Non-uniform inlet air flow in space and time

The study that follows examines the effects of various idealized fuel distribution non-uniformities on detonation tube thrust performance (specific impulse). Kailasanath, et al. (2000) studied the performance effects of unfueled purge fraction, alternately 
referred to as partial fill, showing an increase in $\mathrm{I}_{\mathrm{sp}}$ with increasing purge fraction (decreasing fill fraction). Their study was conducted using a transversely uniform stoichiometric mixture of ethylene and air in the fueled portion of the tube, leaving the exit end of the tube unfueled. No spatial transition (gradient) in equivalence ratio was used between the two regions. Purge fractions of up to $80 \%$ were studied. This current study builds upon and expands the consideration of fuel distribution effects by looking at the effects of equivalence ratio gradients in both the axial and transverse directions as well as comparable partial fill cases. The numerical results are intended to be comparative in nature, looking for trends and relative magnitude effects between the different cases. The actual values of performance are not validated by test data, and so may be subject to different levels of offset error. However, it is expected that the physics-based numerical modeling techniques employed will provide correct insight into the relative performance effects of the different fuel distributions.

\subsection{Paper Organization}

After briefly introducing the basic concept and operation of a PDE in the preceding sections, this thesis presents the specific geometry, operational conditions, and boundary conditions being addressed in the computational study, including all the unique formulations added to the existing SPARK code. The SPARK code itself is then briefly described, along with the basic implementation of the computational grids used in the study. Next, the test cases used to check the accuracy of SPARK for calculating $\mathrm{H}_{2} /$ air detonations are described and the corresponding results presented.

With the code thus validated, the results of the various cases used for the study are presented and compared. These results are then summarized and appropriate conclusions drawn. Lastly, an appendix is provided justifying the selection of the chemistry model chosen for this study.

\section{Problem Formulation}

\subsection{Geometry}

The geometry of a detonation tube is generally simple outside of the transition section between the initiator tube and the main detonation chamber. While the geometry of the area transition is critical to operability of the device, this study will be confined to the constant-area main detonation chamber. This region represents the majority of the volumetric capacity and therefore energy release within the device, making it the dominant region in terms of thrust performance. To keep the geometry as simple as possible, the left hand side inflow plane (main air inlet valves - Figure 1) will be modeled as a solid wall, simulating a transitioned detonation with the inlet valves closed. 
Typical proof-of-concept PDE detonation tubes being used today are in the range of 5 to $12 \mathrm{~cm}$ in diameter and 120 to $180 \mathrm{~cm}$ in overall length (including the initiator tube). For this study a height of $3.0 \mathrm{~cm}$ and a length of $30.0 \mathrm{~cm}$ will be used in order to limit the computational time required. This basic two-dimensional geometry is shown in Figure 4.

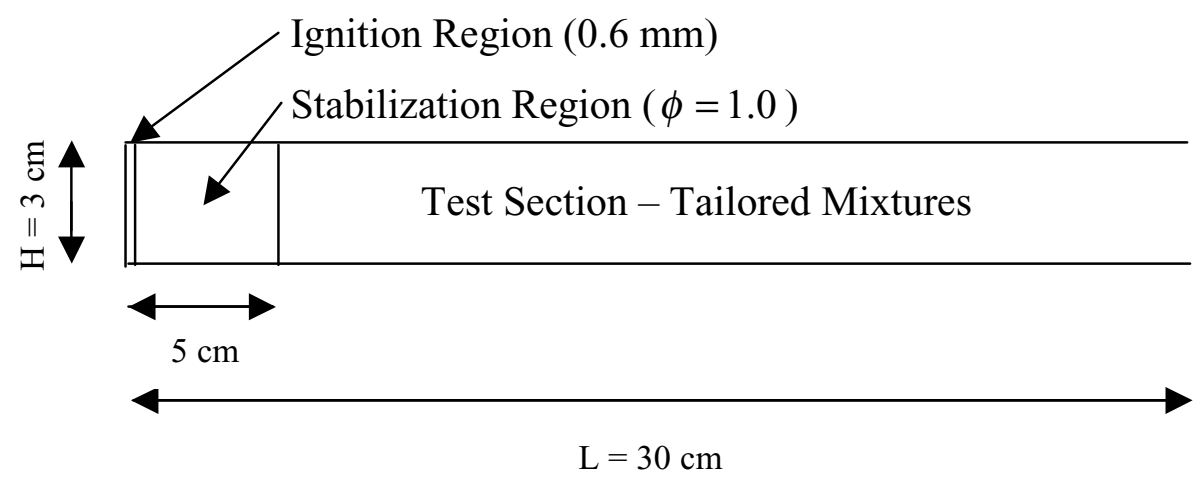

Figure 4 - Geometry for Numerical Study

\subsection{Simplifying Assumptions}

A number of simplifying assumptions are made in this study relative to the actual PDE cycle, as follows.

\subsubsection{DDT}

No attempt is made to model the deflagration to detonation transition (DDT) process, as the phenomena under study is primarily concerned with the steady state detonation propagation. As shown in Figure 4, the initiator tube itself is not modeled nor is the area change from the initiator to the main chamber modeled. A successfully transitioned detonation wave is numerically initiated through the imposition of a high temperature, high pressure ignition zone at the left-hand (inflow) side of the domain in a $5.0 \mathrm{~cm}$ initiation/stabilization region. The high pressure, high temperature ignition zone is set uniformly to 150 atmospheres, $4000 \mathrm{~K}$, and is the full height of the tube, but only $0.6 \mathrm{~mm}$ long, with a composition of $25.5 \% \mathrm{H}_{2} \mathrm{O}$ and $74.5 \% \mathrm{~N}_{2}$ by weight. The total energy contained in the ignition region is $335 \mathrm{~J}$, which is approximately $1.3 \%$ of the heat release from the full $30 \mathrm{~cm}$ detonation tube when it is uniformly, stoichiometrically fueled $(26.2 \mathrm{KJ})$. The remaining $4.94 \mathrm{~cm}$ stabilization region is maintained as a uniform, stoichiometric unburned mixture for each case to aid in establishing a successful detonation regardless of test case mixture properties. Although this detonation may be initially overdriven to insure propagation, it falls back to near the equilibrium ChapmanJouget condition by the end of the stabilization zone. 


\subsubsection{Partial Cycle}

Only the detonation propagation and blowdown portions of the full PDE cycle, portions (4) and (5) in Figure 3, are modeled. It is assumed that the purge and refresh cycles are identical for each case. While this might not be rigorously true in practice, such differences would be expected to be of significantly less importance. It is further assumed that the blowdown of the tube is complete when the tube exit velocity reaches zero anywhere along the exit plane. In a real cycle, some backflow is likely to occur due to the sub-ambient pressure in the tube from the last reflected expansion wave. This portion of the cycle is important when modeling the filling of the tube, both in terms of the fill time and the performance penalty, but this issue is beyond the scope of this study.

\subsection{Boundary Conditions}

The left-hand, top and bottom walls of the two-dimensional detonation tube are all treated as impermeable, dictating that the pressure derivative, the species concentration derivatives, and the normal component of velocity be set to zero at each wall. No-slip wall and adiabatic conditions are set at each surface by setting the temperature derivative and parallel component of velocity to zero. The adiabatic wall condition was selected to simplify the analysis by not requiring resolution of the boundary layer region, however the no-slip wall was maintained to better capture any wave reflections that might occur.

The tube exit boundary conditions for this type of calculation have been a source of considerable study, with various approaches having been used by different individuals, such as Kailasanath and Patnaik (1999) and Wilson and Paxson (2001). Over the course of each cycle, there are six segments of time that need to be addressed when considering the outflow boundary conditions.

1. During the wave propagation segment, the detonable mixture between the detonation wave and the tube exit is undisturbed by the approaching detonation wave. In all of the cases considered in this study, the tube fill pressure matches the ambient pressure, so there is no outflow during this time segment. In most, if not all, real cases, there will be some low velocity flow into or out from the tube.

2. As the detonation wave passes out of the tube, the flow at the exit plane quickly accelerates to a supersonic condition, as the flow between the leading shock and the CJ point is characteristically supersonic.

3. After this short period of supersonic flow, the high pressure behind the reaction zone chokes the flow at the tube exit (Mach number equal to 1).

4. The flow remains choked until a region of supersonic flow generated by reflecting gas dynamic waves reaches the tube exit, causing the exit flow from the tube to be supersonic for a second time.

5. The flow then returns to the choked flow condition until the pressure has dropped sufficiently for the flow to become subsonic.

6. Subsonic outflow throughout the rest of the blowdown. 
Figure 5 shows this sequence of events at the detonation tube centerline from the baseline stoichiometric $\mathrm{H}_{2}$ /air detonation calculation (Case 1) described in detail in Section 5.

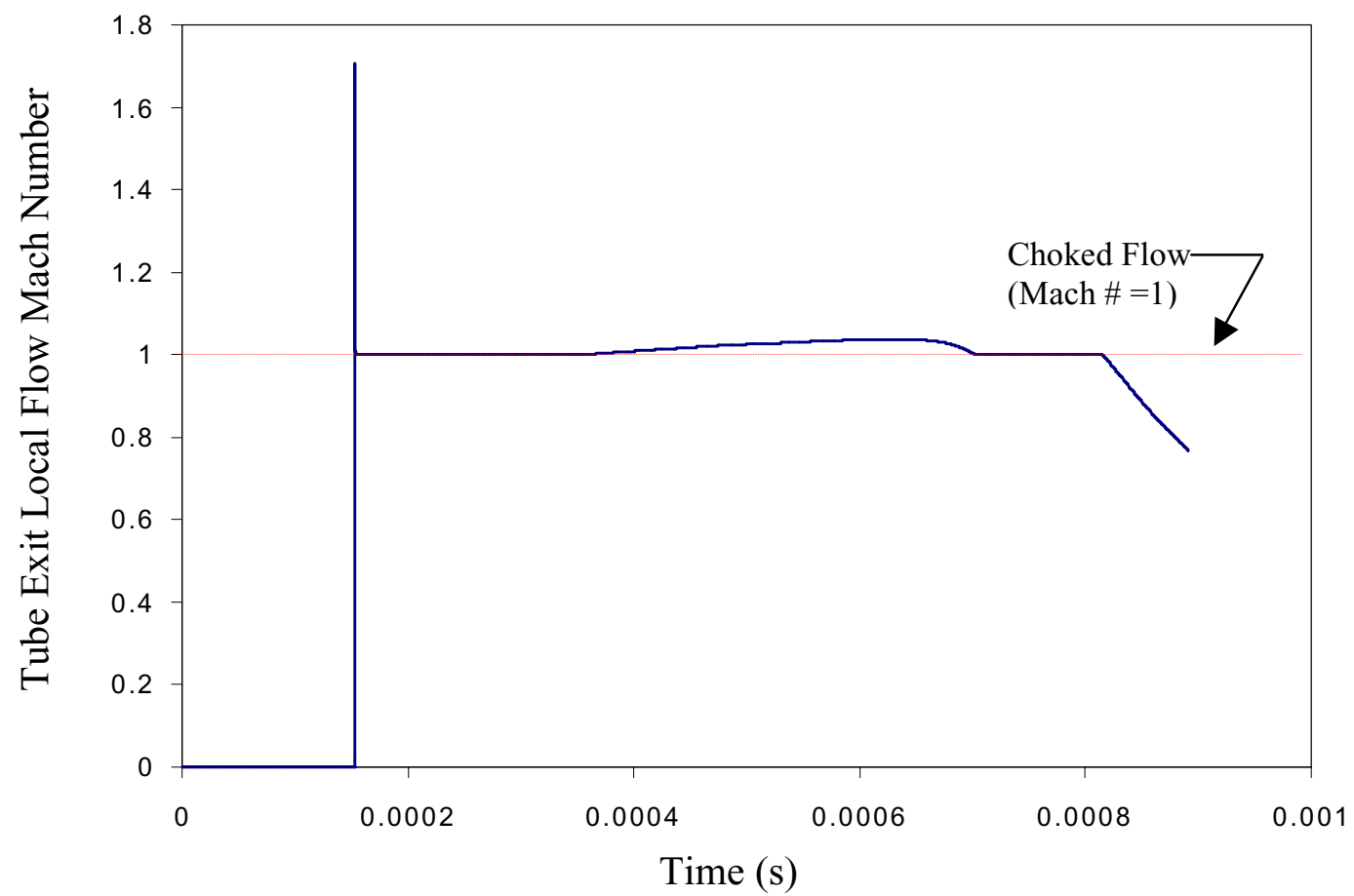

Figure 5 - Time History of Detonation Tube Exit Flow Mach Number at the Tube Centerline

This result is consistent with the results of Wilson and Paxson (2001) from their 1-dimensional calculations.

The outflow boundary conditions required for each of these situations were formulated as follows.

\subsubsection{Subsonic Outflow}

For subsonic outflow, the methodology of Poinsot and Lele (1992) for subsonic reflecting outflows for the Navier-Stokes equations was adopted, from which the following notation is adapted.

First, the basic characteristic velocity terms are calculated from the existing values at the boundary, with the downstream direction defined as the direction of flow.

$\lambda_{1}=u-c:$ The characteristic velocity of the upstream traveling wave 
$\lambda_{2}=u:$ The characteristic convection velocity

$\lambda_{3}=u$ : The characteristic velocity at which the y velocity component is

advected downstream

$\lambda_{5}=u+c:$ The characteristic velocity of the downstream traveling wave

Next, terms associated with the variation of the wave amplitude $\left(\frac{\partial p}{\partial t}-\rho c \frac{\partial u}{\partial t}\right)$ for each velocity term are calculated using the 1-dimensional Euler equations. The inviscid Euler equations are the best available approximation for the viscous (Navier-Stokes) case, and insure compatibility between the physical boundary conditions being calculated and the amplitudes of waves crossing the boundary.

$L_{1}=-L_{5}-2 \frac{d p}{d t}$ : The amplitude term for the characteristic upstream traveling wave

$L_{2}=\lambda_{2}\left(c^{2} \frac{\partial \rho}{\partial X}-\frac{\partial p}{\partial X}\right):$ The amplitude term for the wave associated with the characteristic convection velocity

$L_{3}=\lambda_{3}\left(\frac{\partial v}{\partial X}\right):$ The amplitude term for the wave associated with the

characteristic velocity at which the y velocity component is advected downstream

$L_{5}=\lambda_{5}\left(\frac{\partial p}{\partial X}+\rho c \frac{\partial u}{\partial X}\right)$

The amplitude term for the characteristic downstream traveling wave

The $\lambda_{4}$ and $L_{4}$ terms are omitted as they are used in reference to the 3 rd spatial dimension, not required for this analysis.

Next, the following collection of derivatives at the boundary are cast in terms of the wave amplitude associated terms for use in simplifying the governing equations.

$$
\begin{aligned}
& \frac{\partial(\rho u)}{\partial X}=\frac{1}{c^{2}}\left[L_{2}+\frac{1}{2}\left(L_{5}+L_{1}\right)\right]=d_{1} \\
& u \frac{\partial u}{\partial X}+\frac{1}{\rho} \frac{\partial p}{\partial X}=\frac{1}{2 \rho c}\left(L_{5}-L_{1}\right)=d_{3}
\end{aligned}
$$


$u \frac{\partial v}{\partial X}=L_{3}=d_{4}$

The derivatives $d_{2}$, associated with the energy equation, and $d_{5}$, associated with the third spatial dimension, are not required for the subsonic reflecting outflow case and are therefore omitted here.

Lastly, these derivatives are used in the reformulated version of the 2-dimensional Navier-Stokes equations below to advance the desired quantities to the next time step at the tube exit, assuming constant tangential stresses at the boundary.

$\frac{\partial \rho}{\partial t}+d_{1}+\frac{\partial(\rho v)}{\partial Y}=0 \quad$ (continuity equation)

$\frac{\partial(\rho u)}{\partial t}+u d_{1}+\rho d_{3}+\frac{\partial(\rho u v)}{\partial Y}=\frac{\partial \tau_{11}}{\partial X} \quad(\mathrm{X}$-momentum equation $)$

$\frac{\partial(\rho v)}{\partial t}+v d_{1}+\rho d_{4}+\frac{\partial\left(\rho v^{2}\right)}{\partial Y}+\frac{\partial p}{\partial Y}=\frac{\partial \tau_{22}}{\partial Y} \quad$ (Y-momentum equation $)$

where

$\tau_{11}=\mu\left[2 \frac{\partial u}{\partial X}-\frac{2}{3}\left(\frac{\partial u}{\partial X}+\frac{\partial v}{\partial Y}\right)\right]$

and

$\tau_{22}=\mu\left[2 \frac{\partial v}{\partial Y}-\frac{2}{3}\left(\frac{\partial u}{\partial X}+\frac{\partial v}{\partial Y}\right)\right]$

By first solving for the density using equation (12), the $u$ and $v$ velocity terms can then be found using equations (13) and (14), respectively. For subsonic outflow, the static pressure is known, and is taken to be constant at 1 atmosphere for all the cases run during this study. Wilson and Paxson (2001) have shown that for tubes with a length/diameter (L/D) ratio greater than approximately 10 , this is a valid assumption. This is because the residual effects of the blast wave on the static pressure in the vicinity of the tube exit have dissipated by the time the detonation tube unchokes during the last part of the blowdown, provided the tube pre-detonation pressure is approximately equal to the ambient static pressure. Lastly, the temperature at the exit boundary is determined by extrapolation from interior points assuming a constant local heat flux normal to the tube exit boundary. 


\subsubsection{Supersonic Outflow}

The values of $p, T, u$, and $v$ are linearly extrapolated from the 2 immediate interior points, as described by Anderson (1995). The density, $\rho$, is then calculated using the equation of state.

\subsubsection{Choked Outflow}

The values of $p, T, \rho$ and $v$ are handled as in the supersonic outflow case, but with $u$ limited to the local speed of sound.

\subsection{Detonation Tube Performance}

There are two fundamental methods for calculating the impulse generated by the detonation tube, as taken from Oates (1984). The first is to integrate the pressure along the left hand wall over the partial cycle modeled, subtracting the frictional pressure loss along the two side walls,

$$
I=\int_{0}^{t}\left[\left(\int_{0}^{H} p d Y\right)_{\text {left }}-\left(\left.\int_{0}^{L} \mu \frac{\partial u}{\partial Y}\right|_{\text {wall }} d X\right)_{\text {top }}-\left(\left.\int_{0}^{L} \mu \frac{\partial u}{\partial Y}\right|_{\text {wall }} d X\right)_{\text {bottom }}\right] d t
$$

The second method is to integrate the momentum flux two-dimensionally at the tube exit over the partial cycle, correcting for non-ideal expansion,

$$
I=\int_{0}^{t}\left[\int_{0}^{H} \rho u_{L}^{2} d Y-\int_{0}^{H}\left(p_{0}-p_{L}\right) d Y\right] d t
$$

Both methods, shown per unit width of detonation chamber, will be implemented in the computer code and the relative results will be compared. The calculated impulse can then be used to calculate the specific impulse of the detonation tube.

$$
I_{s p}=\frac{I}{W_{f u e l}}
$$

where $W_{\text {fuel }}$ is the weight of fuel in the tube for one cycle. 


\subsection{Fuel-Air Combinations}

A stoichiometrically fueled $5.0 \mathrm{~cm}$ detonation initiation section is used to provide a stabilized detonation wave to the test section, as described in Section 2.2. The test section equivalence ratio is varied in either the $\mathrm{X}$-direction or the $\mathrm{Y}$-direction from case to case. Three test section average equivalence ratios, alternately denoted as $\Phi$ or "phi", are used, one stoichiometric, one fuel-lean, and one fuel rich. Non-stoichiometric equivalence ratios of 0.9 and 1.1 were selected, representative of moderate levels of nonuniformity, but still well within expected stable detonation limits throughout the detonation tube. Different buffers or gradients of fuel/air mixture are used to achieve the desired average test section equivalence ratio. Transverse gradient cases are representative of a tube centerline fuel injection configuration, while axial gradient cases are representative of a non-constant tube filling process brought on by time varying tube inlet and exit conditions. Partial fill cases are representative of fuel lead $\left(\mathrm{H}_{2}\right.$ buffer $)$ or fuel lag (air buffer) injection timing and provide a point of comparison to the previously referenced work of Kailasanath, et al. (2000). Baseline uniformly fueled cases are used to normalize the results of the subsequent cases. The test cases are grouped as follows, with $\Phi$ referencing the test section average equivalence ratio and $\phi$ referencing the local equivalence ratio within the test section.

$\underline{\text { Stoichiometric Combustion }}(\Phi=1.00)$

Case 1 - Baseline - uniformly fueled throughout test section

Case 2 - Linear transverse gradient $-\phi=1.10$ at the centerline; $\phi=0.90$ at each wall

Case 3 - Linear axial gradient - $\phi=1.10$ at the closed end (after the initial stabilization zone); $\phi=0.90$ at the open end

Fuel-Lean Combustion $(\Phi=0.90)$

Case 4 - Baseline - uniformly fueled throughout test section

Case 5 - Linear transverse gradient $-\phi=1.00$ at the centerline; $\phi=0.80$ at each wall

Case 6 - Linear axial gradient $-\phi=1.00$ at the closed end (after the initial stabilization zone); $\phi=0.80$ at the open end

Case $7-2.50 \mathrm{~cm}$ air buffer at the end of the test section; $\phi=1.00$ up to the buffer air in the test section

$\underline{\text { Fuel-Rich Combustion }}(\Phi=1.10)$

Case 8 - Baseline - uniformly fueled throughout test section

Case 9 - Linear transverse gradient - $\phi=1.20$ at the centerline; $\phi=1.00$ at each wall

Case 10 - Linear axial gradient - $\phi=1.20$ at the closed end (after the initial stabilization zone); $\phi=1.00$ at the open end

Case $11-0.07 \mathrm{~cm} \mathrm{H}_{2}$ buffer at the end of the test section; $\phi=1.00$ up to the buffer $\mathrm{H}_{2}$ in the test section 
Figures 6 and 7 show graphically the different axial and transverse fuel distribution schemes to be used in the "test section" of the detonation tube, excluding the baseline uniformly fueled cases (Cases 1, 4, and 8) and the partial-fill cases (Cases 7 and 11).

Test cases performed using the opposite orientation of fuel distribution nonuniformity (transverse distributions that were fuel lean at the centerline and fuel rich at the walls, and axial distributions that increased in equivalence ratio along the length of the tube instead of decreasing) yielded nearly identical detonation tube performance results as the cases shown above, and so were not included in this study.

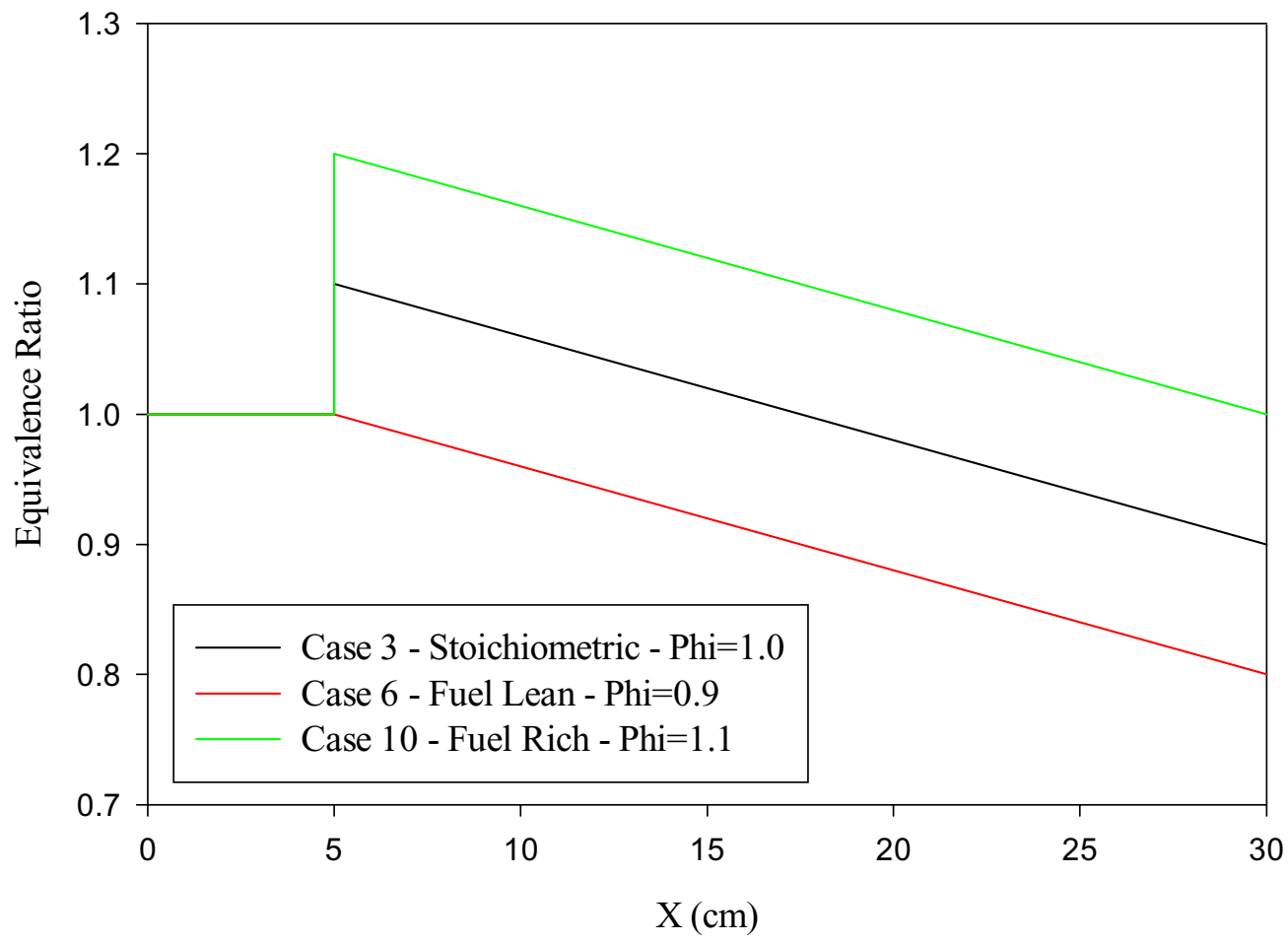

Figure 6 - Test Section Axial Fuel Distributions 


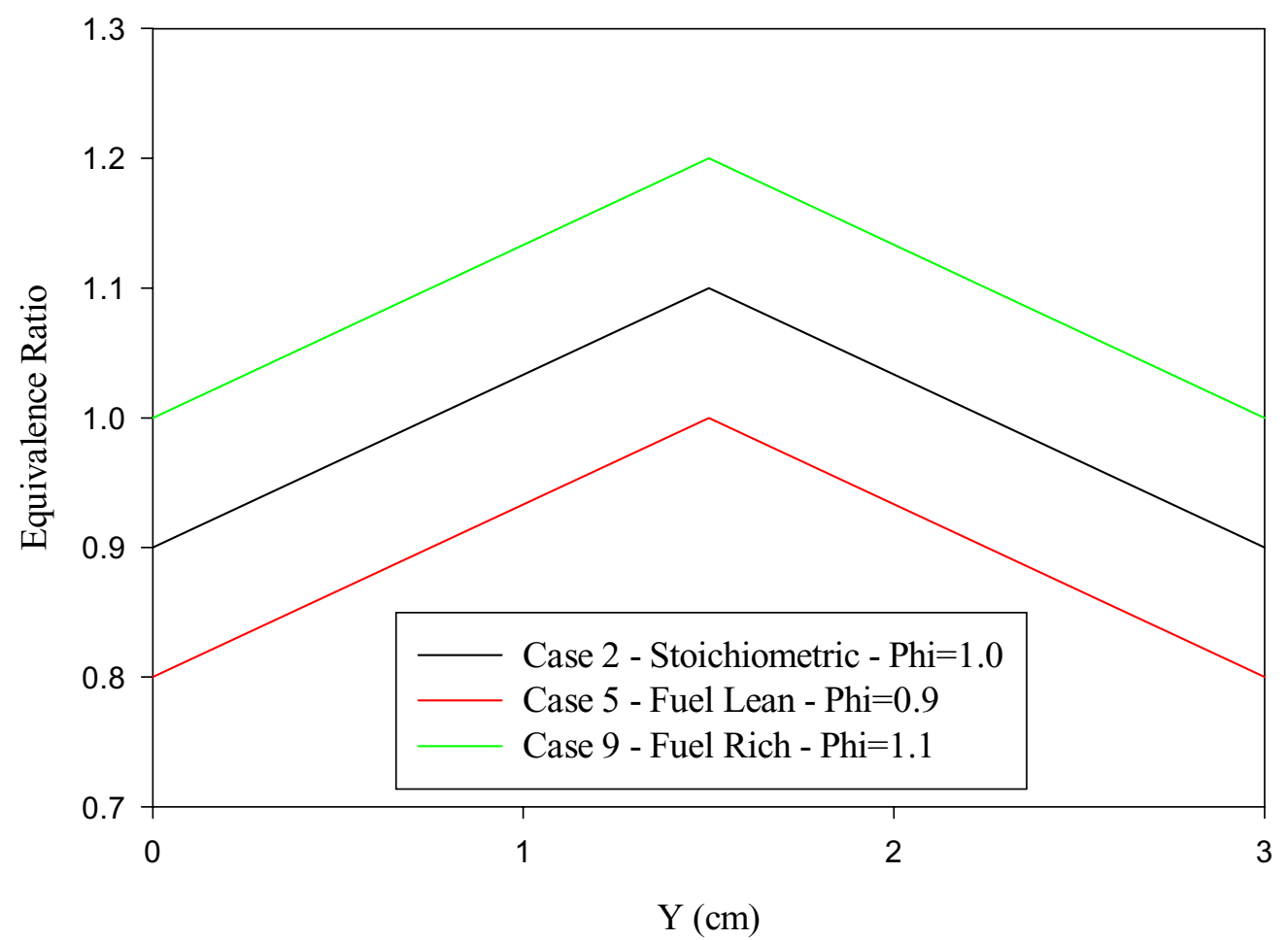

Figure 7 - Test Section Transverse Fuel Distributions

\section{Numerical Tool Description}

The numerical tool chosen for this study was the SPARK 2D Navier-Stokes Code developed at the NASA Langley Research Center. The SPARK code was developed primarily for the study of high speed reacting flows, particularly those found in scramjet engines, under the National AeroSpace Plane (NASP) program. SPARK is a versatile time accurate code that has been used in a number of supersonic combustion and detonation studies (Drummond and Mukunda, 1988; Drummond, 1988; Carpenter, 1989). The code has been previously described in detail by Drummond (1990), so this description will be limited to a general overview of the principal features salient to this study.

\subsection{Governing Equations}

SPARK uses the strong conservation form of the Navier-Stokes, energy, and species continuity equations as derived by Williams (1985). 
Continuity:

$\frac{\partial \rho}{\partial t}+\nabla \cdot(\rho \overrightarrow{\mathrm{V}})=0$

Momentum:

$\frac{\partial(\rho \vec{V})}{\partial t}+\nabla \cdot(\rho \vec{V} \vec{V})=\nabla \cdot \tau+\rho \sum_{i=1}^{n s} f_{i} \vec{b}_{i}$

where $\tau$ is here defined as

$\tau=\tau_{i j}=-\delta_{i j} p+\mu\left(\frac{\partial u_{i}}{\partial x_{j}}+\frac{\partial u_{j}}{\partial x_{i}}\right)$

Energy:

$\frac{\partial(\rho E)}{\partial t}+\nabla \cdot(\rho \vec{V} E)=\nabla \cdot(\tau \cdot \vec{V})-\nabla \cdot \vec{q}+\rho \sum_{i=1}^{n s} f_{i} \vec{b}_{i}\left(\vec{V}+\vec{V}_{i}\right)$

where

$$
\begin{aligned}
& \vec{q}=-k \nabla T+\rho \sum_{i=1}^{n s} h_{i} f_{i} \vec{V}_{i}+R_{u} T \sum_{i=1}^{n s} \sum_{j=1}^{n s}\left(\frac{X j D_{T i}}{M_{i} D}\right)\left(\vec{V}_{i}-\vec{V}_{j}\right) \\
& E=\sum_{i=1}^{n s} h_{i} f_{i}-\frac{p}{\rho}+\frac{u^{2}+v^{2}}{2}
\end{aligned}
$$

and

$\vec{V}_{i}=-\frac{D}{f_{i}}\left(\frac{\partial f_{i}}{\partial X} \hat{x}+\frac{\partial f_{i}}{\partial Y} \hat{y}\right)$

Species Continuity:

$\frac{\partial\left(\rho f_{i}\right)}{\partial t}+\nabla \cdot\left(\rho \vec{V} f_{i}\right)=w_{i}-\nabla \cdot\left(\rho f_{i} \vec{V}_{i}\right)$ 
The expression for diffusion velocity, $\vec{V}_{i}$, assumes that $\mathrm{N}_{2}$ is the major species present, with all other species present in "trace" amounts. It can then be assumed that only the diffusion of each species with $\mathrm{N}_{2}$ be considered, and further that all binary diffusion coefficients are equal, as described by Drummond (1990).

Anderson (1995) has pointed out that when the governing equations are cast in the strong conservation form as above, there is no issue with capturing shocks in the flow field. Since the equations are set up in terms of fluxes instead of the primitive variables $(\rho, u, v, p, e t c$.$) , there are no discontinuities across shock waves within the governing$ equations that require separate consideration for closure.

\subsection{Detailed Chemistry}

Following the work of Cambier and Adelman (1988) and Lynch and Edelman (1994), a 7-step, 7-species hydrogen-air detailed chemical mechanism was chosen for this study instead of the more standard 18-step, 9-species mechanism of Jachimowski (1988). For computational efficiency, it is desirable to utilize the simplest possible mechanism that can with reasonable accuracy provide the correct time evolution and chemical composition of the detonation wave and the resultant combustion products. The 7-step mechanism is a simple reduction of the 18-step mechanism through the elimination of $\mathrm{HO}_{2}$ and $\mathrm{H}_{2} \mathrm{O}_{2}$ from the chemical system, leaving $\mathrm{H}_{2}, \mathrm{O}_{2}, \mathrm{H}_{2} \mathrm{O}, \mathrm{OH}, \mathrm{H}, \mathrm{O}$, and $\mathrm{N}_{2}$ (inert). This simplification can be justified when it is considered that in the basic ZND detonation model, ignition occurs after the leading shock, at which point the flow temperature is elevated to a point where the chemistry of $\mathrm{HO}_{2}$ and $\mathrm{H}_{2} \mathrm{O}_{2}$ is no longer dominant. The Appendix provides further analysis to justify the use of the simplified chemical mechanism.

Each chemical reaction is modeled using the standard Arrhenius expression for the forward reaction rate,

$k_{f}=A * T^{n} * e^{\frac{-E a}{R_{u} T}}$

For the 7-step mechanism used in this study the values of $A, n$, and $E a$ are as follows. 
Table 1 - 7-Step $\mathrm{H}_{2}$-Air Mechanism

\begin{tabular}{|l|c|c|c|l|}
\hline $\begin{array}{l}\text { Reaction } \\
\text { Ref. } \#\end{array}$ & Reaction & $\mathrm{A}$ & $\mathrm{n}$ & $\begin{array}{c}\text { Ea } \\
\text { (cal/g-mole) }\end{array}$ \\
\hline 1 & $\mathrm{H}_{2}+\mathrm{O}_{2} \Leftrightarrow 2 \mathrm{OH}$ & $0.1700 \mathrm{e} 14$ & 0.00 & 48150 \\
\hline 2 & $\mathrm{H}+\mathrm{O}_{2} \Leftrightarrow \mathrm{OH}+\mathrm{O}$ & $0.1420 \mathrm{e} 15$ & 0.00 & 16400 \\
\hline 3 & $\mathrm{H}_{2}+\mathrm{OH} \Leftrightarrow \mathrm{H}_{2} \mathrm{O}+\mathrm{H}$ & $0.3160 \mathrm{e} 08$ & 1.80 & 3030 \\
\hline 4 & $\mathrm{O}+\mathrm{H}_{2} \Leftrightarrow \mathrm{OH}+\mathrm{H}$ & $0.2070 \mathrm{e} 15$ & 0.00 & 13750 \\
\hline 5 & $\mathrm{OH}+\mathrm{OH} \Leftrightarrow \mathrm{H}_{2} \mathrm{O}+\mathrm{O}$ & $0.5500 \mathrm{e} 14$ & 0.00 & 7000 \\
\hline 6 & $\mathrm{OH}+\mathrm{H}+\mathrm{M} \Leftrightarrow \mathrm{H}_{2} \mathrm{O}+\mathrm{M}$ & $0.2210 \mathrm{e} 23$ & -2.00 & 0 \\
\hline 7 & $\mathrm{H}+\mathrm{H}+\mathrm{M} \Leftrightarrow \mathrm{H}_{2}+\mathrm{M}$ & $0.6530 \mathrm{e} 18$ & -1.00 & 0 \\
\hline
\end{tabular}

The $3^{\text {rd }}$ body efficiencies of all species for reactions 6 and 7 are assumed to be one. The backward rate constant is then found from the simple expression,

$k_{b}=\frac{k_{f}}{K_{e q}}$

where $K_{e q}$ is found using a Gibbs free energy minimization scheme similar to those found in chemical equilibrium codes such as the NASA Glenn Research Center's CEA code developed by Gordon and McBride (1994). The chemical system involving the 6 reactive species is solved at each time step, thus providing the source term for the species equation (27). The mass of nitrogen present is determined by summing the mass fractions of each of the other species and then subtracting the result from the necessary total of 1 . Mass conservation is maintained throughout the computational domain at each time step.

\subsection{Thermodynamic and Transport Properties}

SPARK utilizes previously developed analytical expressions for the required thermodynamic and transport properties used throughout the code, which, as with the previously discussed aspects of the code, have been related in detail in other publications, such as Drummond (1990). Species specific heat at constant pressure and species Gibbs energy are calculated using $4^{\text {th }}$ and $5^{\text {th }}$ order polynomial curve fits of temperature, respectively. The species laminar viscosity, $\mu_{i}$, is calculated using an expression known as Sutherland's law, 
$\mu_{i}=A_{i}\left(\frac{T}{B_{i}}\right)^{1.5} \frac{C_{i}+D_{i}}{T+D_{i}}$,

where $A_{i}, B_{i}, C_{i}$, and $D_{i}$ are constants which vary with species.

The mixture value of viscosity is then calculated using Wilke's law.

$\mu=\sum_{1}^{n s} \frac{\mu_{i}}{\left(1+\frac{1}{X_{i}} \sum_{j=1, j \neq i}^{n s} X_{j} \varphi_{i j}\right)}$

where

$$
\varphi_{i j}=\frac{\left[1+\left(\frac{\mu_{i}}{\mu_{j}} \frac{\rho_{i}}{\rho_{j}}\right)^{0.5}\left(\frac{M_{i}}{M_{j}}\right)^{0.25}\right]^{2}}{\frac{4}{\sqrt{2}}\left(1+\frac{M_{i}}{M_{j}}\right)^{0.5}}
$$

Lastly, the binary diffusion coefficient, $D$, and the thermal conductivity, $k$, are calculated from the Schmidt number (0.22) and Prandtl number (0.72), respectively, which are provided as inputs to the code.

$$
\begin{aligned}
& D=\frac{\mu}{\rho S c}, \\
& k=\frac{C_{p} \mu}{\operatorname{Pr}}
\end{aligned}
$$

All properties were calculated assuming laminar flow throughout.

\subsection{Computational Grid}

Looking back at the detonation structure shown in Figure 2, it is easily seen that the region in the immediate vicinity of the detonation wave front will determine the required physical grid spacing for the computational model. If the goal of this study were to examine the detailed structure of the shock wave coupled to the reaction zone, it would be necessary to provide a computational grid spacing on the order of the width of the leading shock wave (shown by Shapiro (1953) to be on the order of $1.4 \times 10^{-7} \mathrm{~m}$ ), and probably finer. Fortunately, it is not necessary to resolve this structure in order to obtain accurate flow properties required to calculate the performance values that are the goal of 
this study. It is sufficient to provide enough grid resolution to capture the shortest reaction times of the chemical system as the flow behind the shock is convected downstream. For the 7-step chemical system and conditions investigated in this study, this worked out to be on the order of $1 \times 10^{-5} \mathrm{~m}$. Grid spacing significantly larger than this, on the order of $1 \times 10^{-4} \mathrm{~m}$, resulted in oscillations in the combustion zone. When even courser grids were tried, the calculation went completely unstable. From a practical standpoint, it is desirable to use the largest acceptable grid spacing in order to reduce the number of required grid points. Also, since the system of equations being solved are numerically "stiff", Radhakrishnan (1991) has shown that going to a finer grid spacing results in a proportionally smaller required time step, leading to very long computational times.

Since an X-grid spacing of $1 \times 10^{-5} \mathrm{~m}$ would yield 30,000 grid points in the $\mathrm{X}$ direction for each Y-grid location for a $30 \mathrm{~cm}$ tube, it becomes immediately apparent that this grid spacing needs to be confined to the region where it is required, namely the leading section of the detonation wave where steep gradients in all the principal flow and composition variables are present. Since the detonation wave is moving in space, the fine grid must move with it. This was accomplished for this study by starting with a uniform 300-point grid in $\mathrm{X}$ at the fine grid spacing $\left(1 \times 10^{-5} \mathrm{~m}\right)$. As the detonation wave approaches the right-hand edge of the computational domain, the grid is stretched by approximately 30 times the fine grid spacing at the left hand side where the reaction has already occurred, lengthening the overall domain in X. The fine grid section and the coarser grid section are connected through a spline fit,

$$
X_{i}=X_{i-1}+\Delta X_{\text {coarse }}-\frac{\left(\Delta X_{\text {coarse }}-\Delta X_{\text {fine }}\right)}{1+e^{-0.05555\left(i-i_{\text {ref }}\right)}},
$$

where $i_{\text {ref }}$ is the center grid point of the spline, which is set to 60 for all the simulations in this study. This value of $i_{\text {ref }}$ gives approximately 100 grid points at the fine grid spacing at the right hand side of the domain. The wave structure is thus always maintained within the fine grid section, which is effectively shifted to the right every time the detonation wave travels 30 times the fine grid spacing. The current result is then interpolated onto the new grid and the calculation is continued. Once the desired length of the full detonation tube is reached, the fine grid is maintained at the right hand (exit) end of the tube. Figure 8 shows the physical X-grid spacing at detonation wave positions (domain lengths) of $3 \mathrm{~cm}, 10 \mathrm{~cm}$, and $30 \mathrm{~cm}$. 


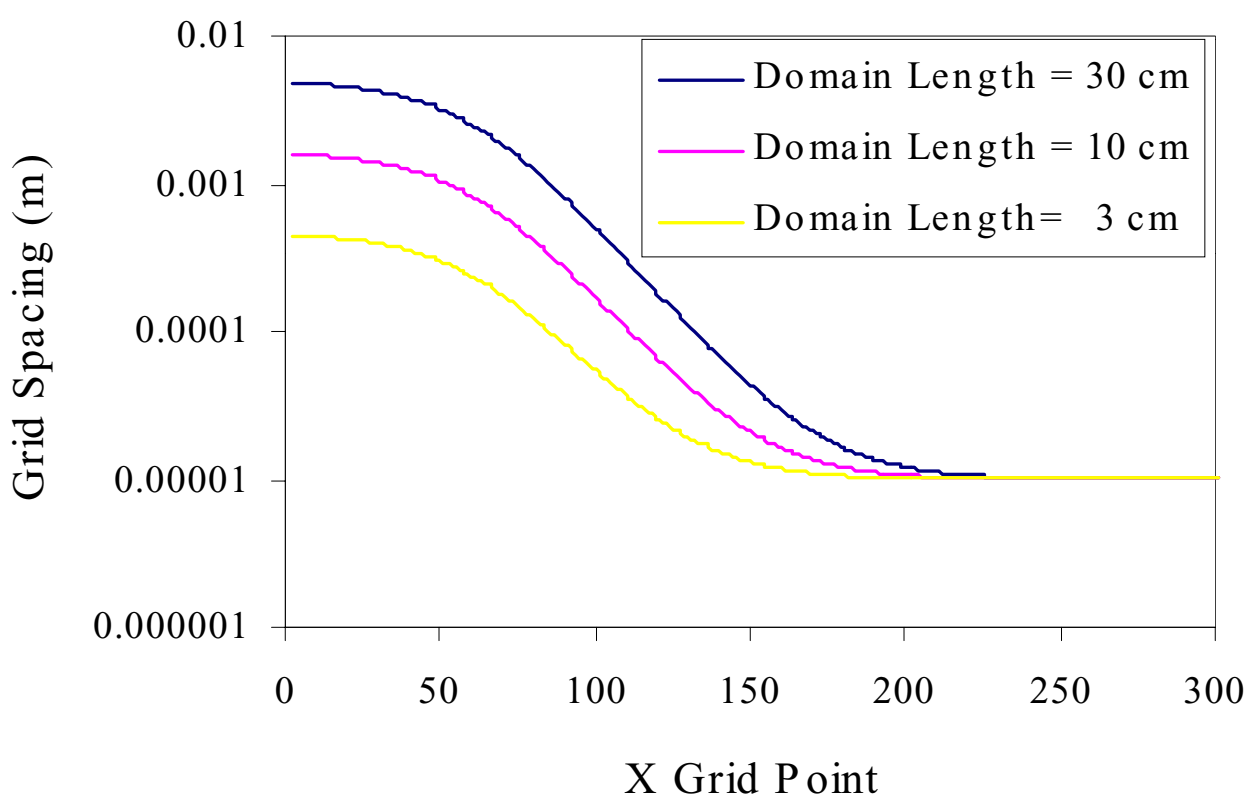

Figure 8 - Variable X-Grid Spacing

The Y-grid spacing remains the same throughout each calculation. Grid points are clustered near the wall in order to capture with reasonable fidelity the severe gradient of velocity generated by the detonation wave in that region. As with the X-grid, significantly finer grid spacing yields proportionally smaller time steps, leading to excessively long computational times. Grid spacings as small as $5 \times 10^{-6} \mathrm{~m}$ did not require time steps smaller than those required for the finite-rate chemistry. The general Y-grid distribution used throughout the study is shown in Figure 9. Minor adjustments to this grid were used case-to-case to minimize run times while maintaining detonation wave stability.

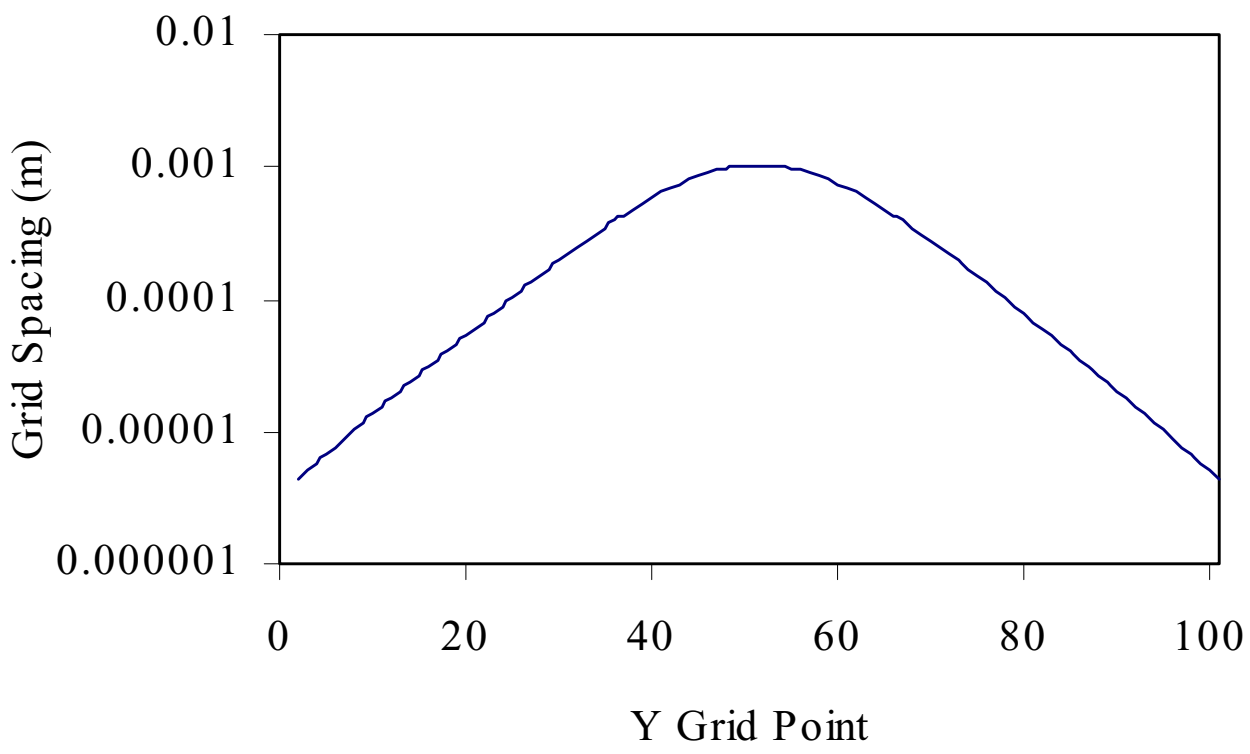

Figure 9 - Y-Grid Spacing 
Once the grid has been generated, the method of Smith and Weigel (1980), referred to as the "two-boundary technique," is used to transform the mesh into a uniform 2 -dimensional boundary-fitted rectangular grid $(\xi, \eta)$ for computation. The disconnected domain boundaries are defined by analytic functions, which are then connected together using parametric polynomials to generate the grid. This transformation allows for more efficient computation of the time-accurate solution, which will be further elaborated on in the next section. The solution is then transformed back into the original physical space for output.

\subsection{Calculation Implementation and Numerical Solution}

Once the geometric domain has been discretized into the computational grid, the governing equations are vectorized for solution in the form,

$\frac{\partial \vec{U}}{\partial t}+\frac{\partial \vec{F}}{\partial X}+\frac{\partial \vec{G}}{\partial Y}=\vec{H}$

where the $\vec{U}$ vector represents the dependant variables, the $\vec{F}$ and $\vec{G}$ vectors the convective and diffusive terms in $\mathrm{X}$ and $\mathrm{Y}$, respectively, and the $\vec{H}$ vector the source terms. In the transformed coordinate system, equation (36) becomes,

$$
\frac{\partial \overrightarrow{\hat{U}}}{\partial t}+\frac{\partial \overrightarrow{\hat{F}}}{\partial \xi}+\frac{\partial \overrightarrow{\hat{G}}}{\partial \eta}=\overrightarrow{\hat{H}}
$$

where

$$
\begin{aligned}
& \overrightarrow{\hat{U}}=J \vec{U}, \\
& \overrightarrow{\hat{F}}=\frac{\partial Y}{\partial \eta} \vec{F}-\frac{\partial X}{\partial \eta} \vec{G}, \\
& \overrightarrow{\hat{G}}=\frac{\partial X}{\partial \xi} \vec{G}-\frac{\partial Y}{\partial \xi} \vec{F}, \\
& \overrightarrow{\hat{H}}=J \vec{H}
\end{aligned}
$$

and where 
$J=\frac{\partial X}{\partial \xi} \frac{\partial Y}{\partial \eta}-\frac{\partial Y}{\partial \xi} \frac{\partial X}{\partial \eta}$

This vectorized equation is then solved using a MacCormack explicit predictorcorrector scheme as described by Drummond et al. (1991), that is $4^{\text {th }}$ order accurate in space and $2^{\text {nd }}$ order accurate in time. While the code allows for both partially and fully implicit schemes to be used, these options were not selected for this study in order to maintain the greatest accuracy possible. The time step was limited so as to allow a maximum change in any species mass fraction of $0.8 \%$ per iteration at any grid point. With these options selected the typical simulation contained in this study required 60 hours to complete on a Silicon Graphics Octane2 workstation with a $400 \mathrm{MHz}$ R12000A processor.

\section{Code Validation}

\subsection{Introduction}

Before beginning to utilize the SPARK code for the configuration and conditions of interest, it is necessary to demonstrate that the code can accurately compute one or more known solutions in similar flow regimes. The SPARK code has been previously validated for such cases as

- Non-reacting

○ Laminar spatially developing shear layer

○ Turbulent spatially developing mixing layer

- Mach 14 laminar 15 degree compression corner

○ Transverse jet behind a rearward facing step

- Reacting

○ $\mathrm{H}_{2}$-air turbulent mixing and reaction

○ Burrows-Kurkov Mach $2.44 \mathrm{H}_{2}$ fueled vitiated scramjet combustor

All of these cases are described in Drummond (1991).

Additionally, prior to initiating the detonation computations included in this study, several standard non-reacting test cases were performed, including a supersonic expansion corner, a supersonic compression corner, and a $\mathrm{H}_{2}-\mathrm{N}_{2}$ shock tube problem. In all cases the results were consistent with the corresponding classical analytical solution. In addition to the above validations, it was necessary to perform validation calculations specifically for detonation. Two cases were selected for which known solutions with detailed kinetic mechanisms exist or could be easily calculated using other validated codes, as follows. 


\section{2 $\quad \mathrm{H}_{2}$-Air Oblique Detonation Wave}

Thaker and Chelliah (1997) used the SPARK code to calculate the detonation structure of an oblique detonation wave created from a Mach 9.3 stoichiometric $\mathrm{H}_{2}$-air mixture flowing over a 27 degree wedge. The geometry and computational domain are shown in Figure 10.

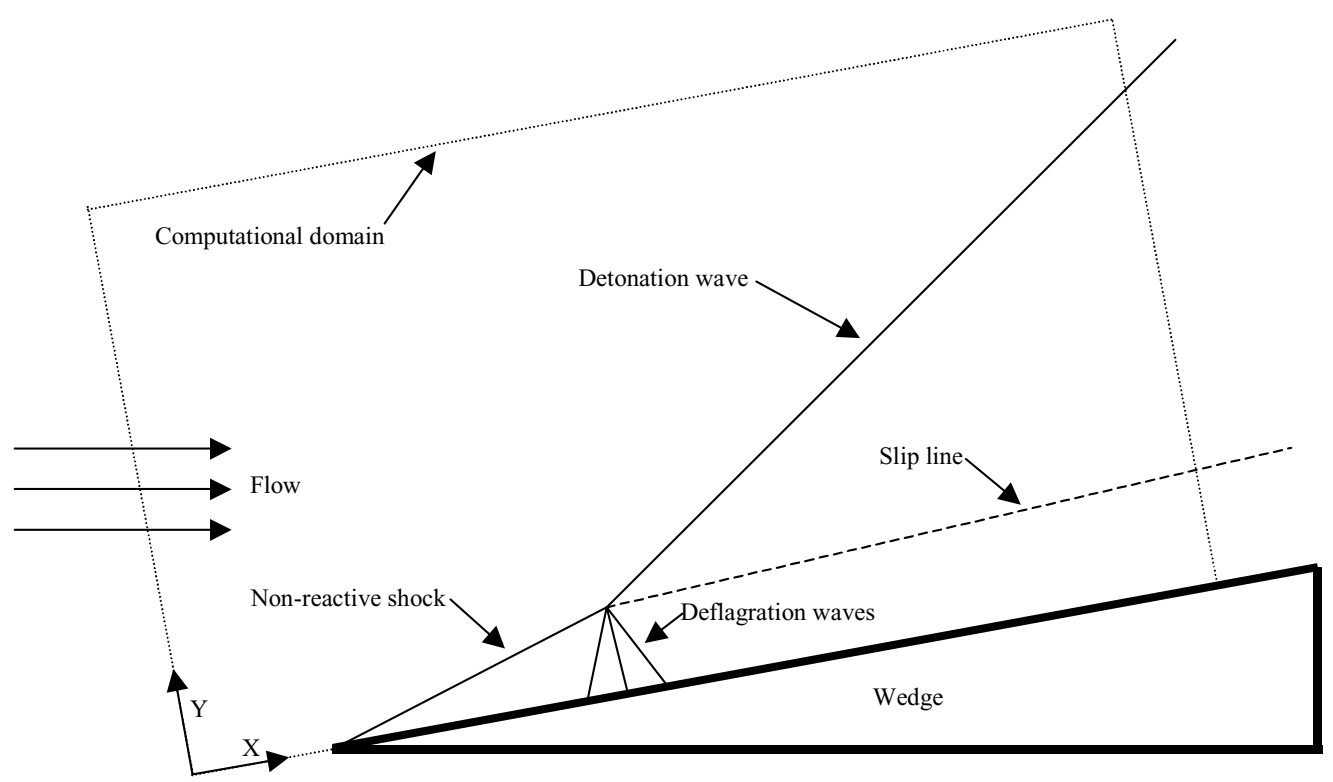

Figure 10 - Oblique Detonation Geometry and Computational Domain

As shown, the computational domain was tilted 27 degrees to coincide with the wedge surface. A 200 by 150 uniform grid was used to model the $5 \mathrm{~mm}$ by $2.5 \mathrm{~mm}$ region. $0.5 \mathrm{~mm}$ of open boundary was included in the lower boundary before the leading edge of the wedge. A slip-wall, adiabatic boundary condition was used along the wedge wall.

The flow entering the domain was at the following conditions,

Pressure $=1$ atmosphere

$\mathrm{X}$-direction velocity $=3430 \mathrm{~m} / \mathrm{s}$

$\mathrm{H}_{2}$ mass fraction $=0.0283$

$\mathrm{N}_{2}$ mass fraction $=0.7452$
Temperature $=300 \mathrm{~K}$

Y-direction velocity $=-1748 \mathrm{~m} / \mathrm{s}$

$\mathrm{O}_{2}$ mass fraction $=0.2265$

The baseline calculation of Thaker and Chelliah (1997) was first repeated using the 12-step $\mathrm{H}_{2}$-air mechanism used in their study. This mechanism was taken from the full 18-step mechanism given in the Appendix by removing the $\mathrm{H}_{2} \mathrm{O}_{2}$ reactions. The resulting temperature and $\mathrm{H}_{2} \mathrm{O}$ mass fraction results are shown in Figure 11 and Figure 12. These results are consistent with those of Thaker and Chelliah (1997). 


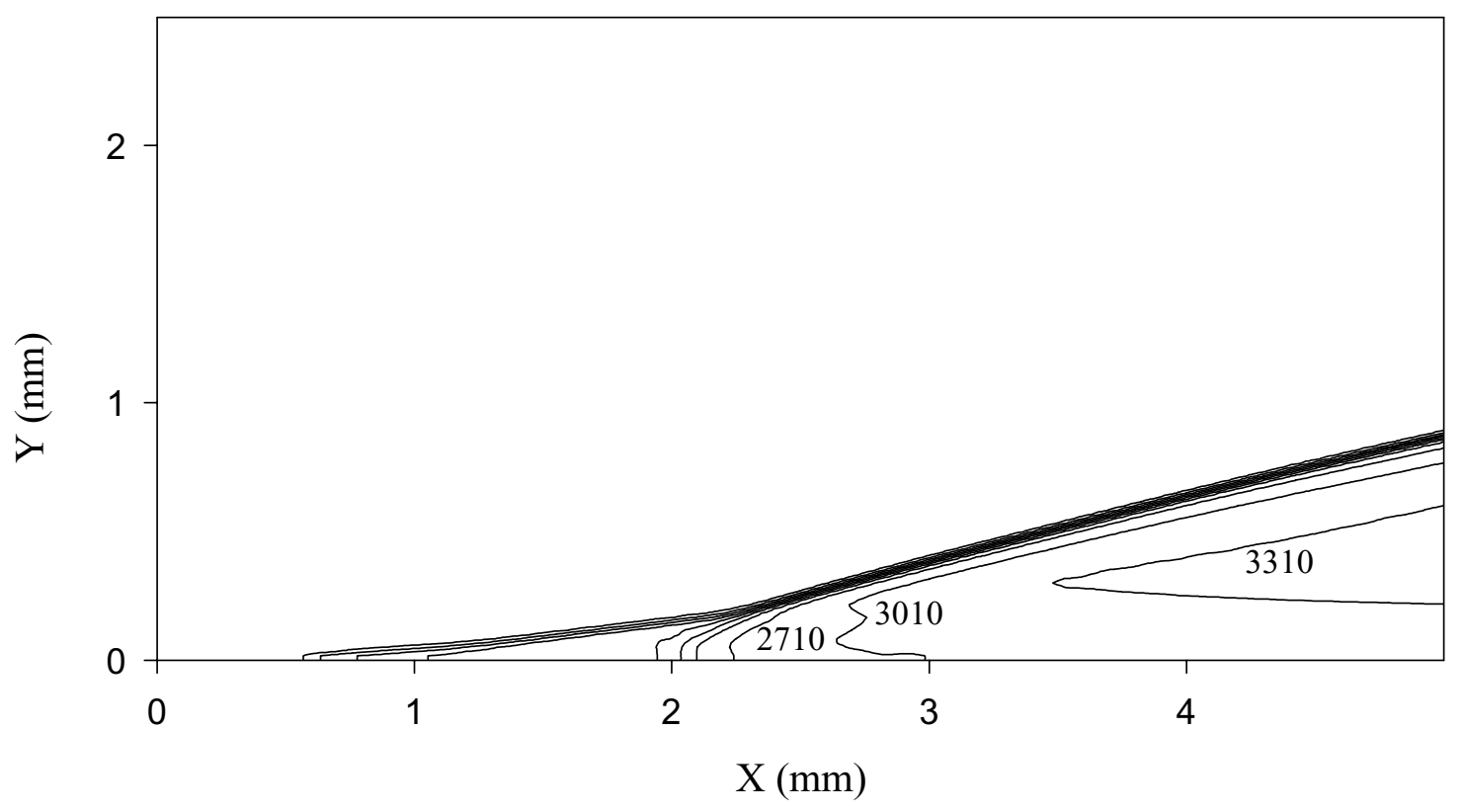

Figure 11 - 12-Step Mechanism Oblique Detonation Temperature Contours

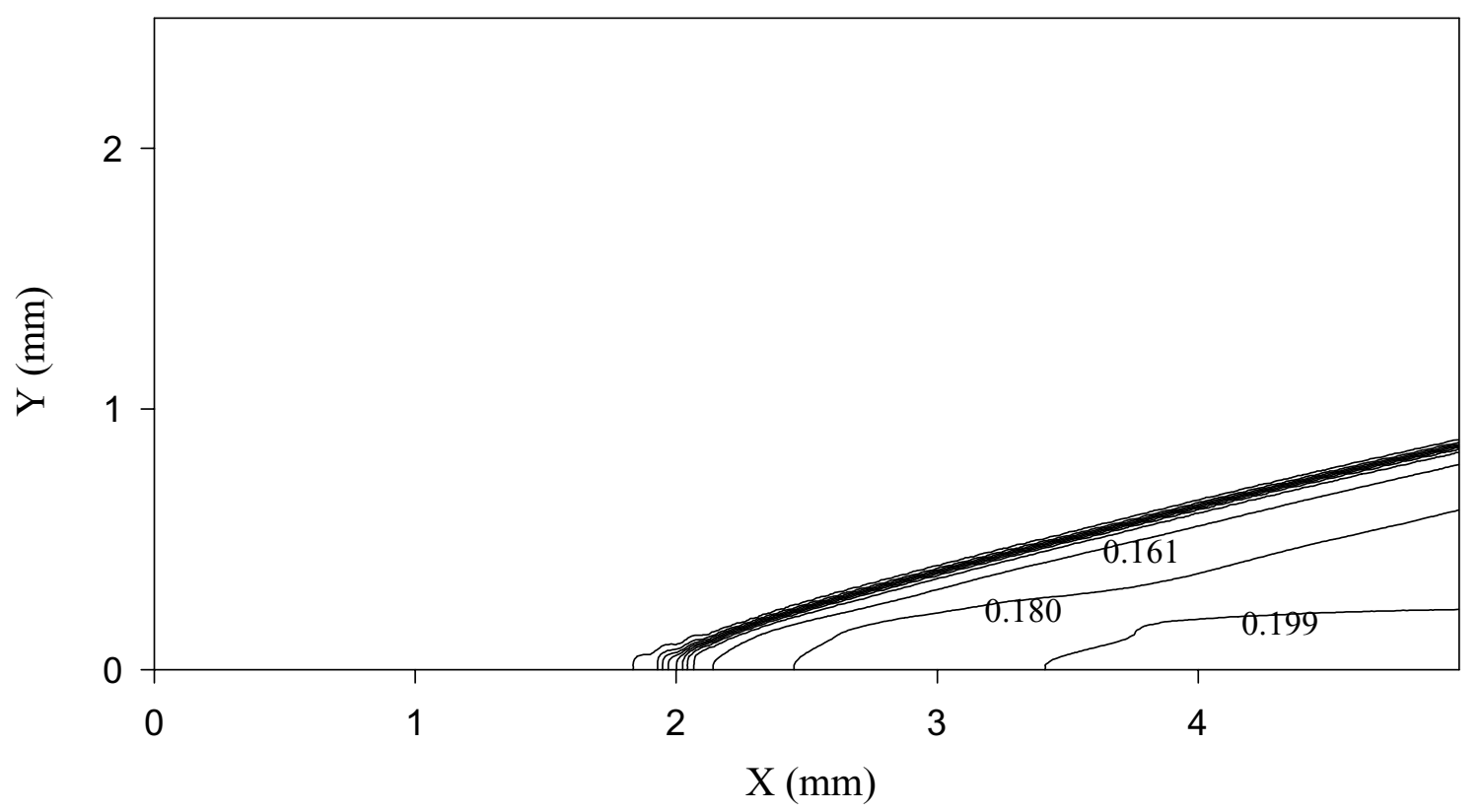

Figure 12 - 12-Step Mechanism Oblique Detonation $\mathrm{H}_{2} \mathrm{O}$ Mass Fraction Contours

Next, the calculation was repeated using the 7-step mechanism chosen for this study. As shown in Figures 13 and 14, the initial shock/reaction zone is very similar to that obtained with the 12-step mechanism. However, it is apparent that the final stages of heat release are somewhat slower with the 7-step mechanism. This is consistent with the 1-D results to be shown in the next section. It is not expected that this slight delay in heat release will influence the comparative results of this study. 


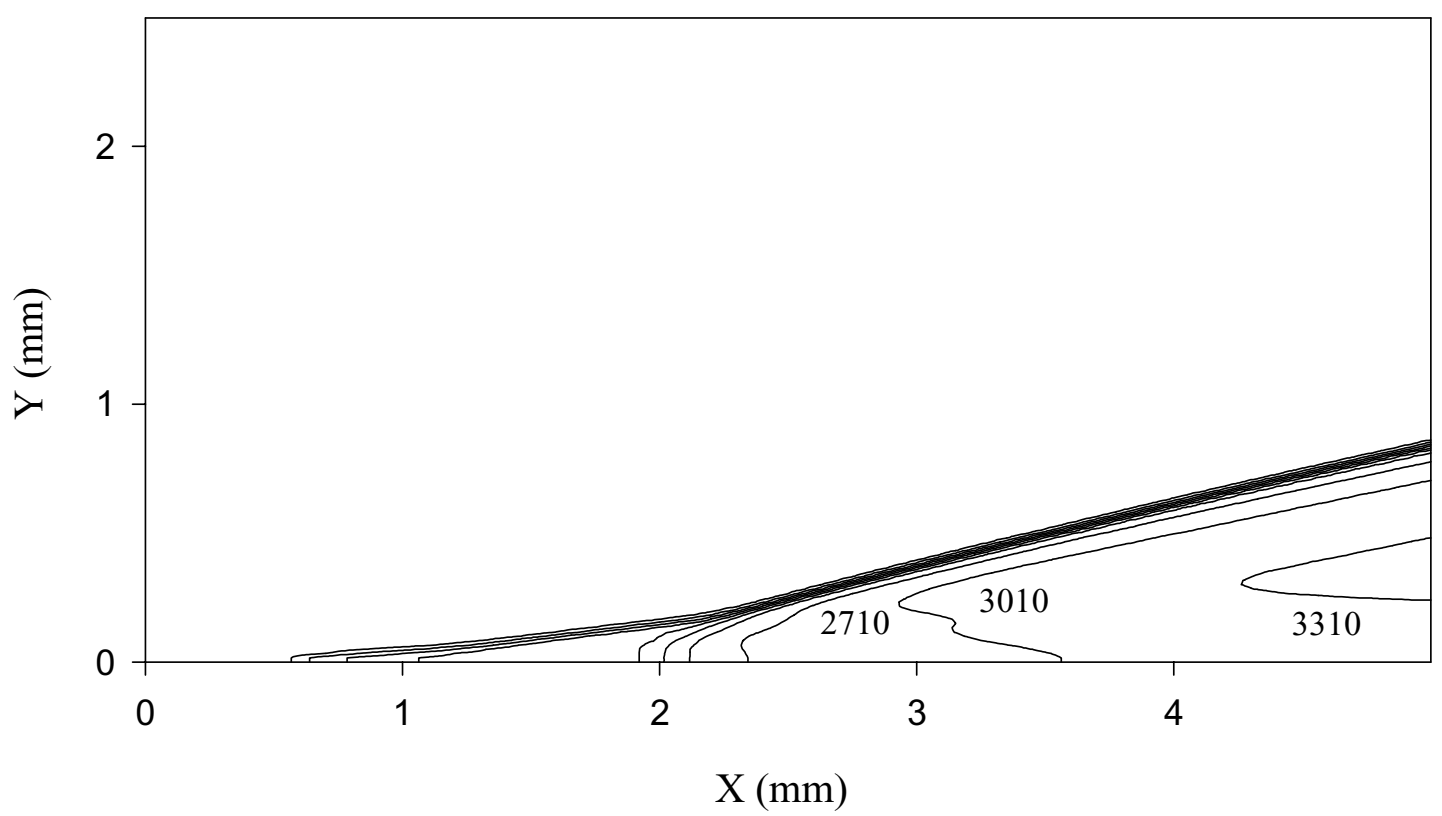

Figure 13 - 7-Step Mechanism Oblique Detonation Temperature Contours

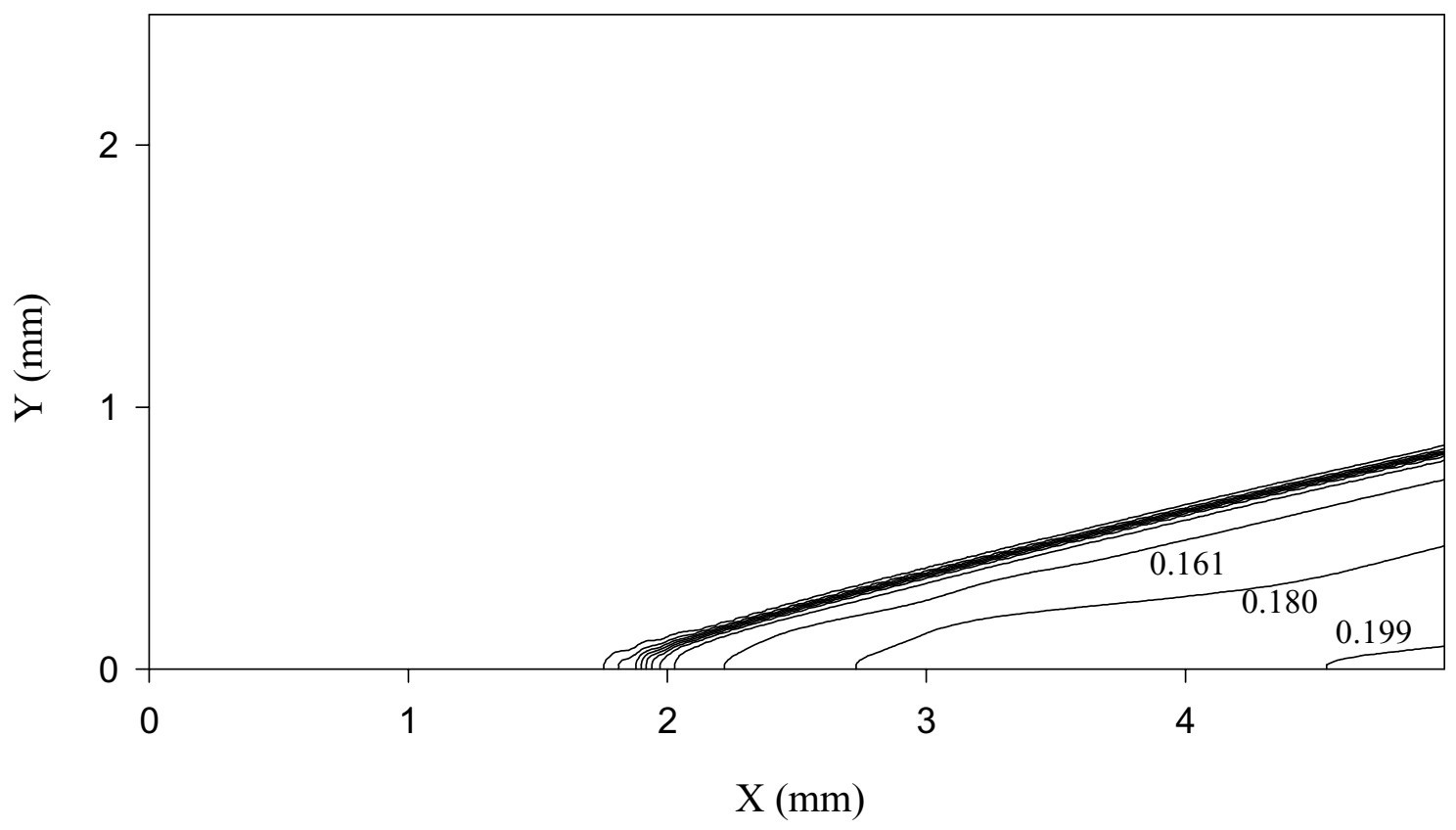

Figure 14 - 7-Step Mechanism Oblique Detonation $\mathrm{H}_{2} \mathrm{O}$ Mass Fraction Contours 


\subsection{Uniform $\mathrm{H}_{2}$-Air Mixture ZND Detonation}

As a final code validation case, the detonation structure computed by SPARK in a uniform stoichiometric $\mathrm{H}_{2}$-air mixture initially at 1 atmosphere pressure and $300 \mathrm{~K}$ (Case 1 of this study, detonation tube centerline results) was compared to that obtained from the 1-dimensional ZND code developed by Shepherd (1986). To run the ZND code, the desired kinetic mechanism is first input to the CHEMKIN code, described by Kee et al. (1989), that provides the kinetic and thermodynamic properties required. Next, the Gordon and McBride (1994) CEA code, or any other equilibrium detonation code, is used to determine the detonation (CJ) velocity. With these inputs, ZND calculates the 1-D detonation structure by first assuming a normal shock based on the detonation velocity and then reacting the mixture via the kinetic mechanism and gas properties from CHEMKIN using the post-shock conditions as the initial conditions. ZND performs these calculations in the reference frame fixed to the detonation wave, shown in Figure 15 , so the velocity must be transformed back to the stationary reference frame by adding the detonation wave velocity. All of the static properties are unaffected by the change in reference frames.

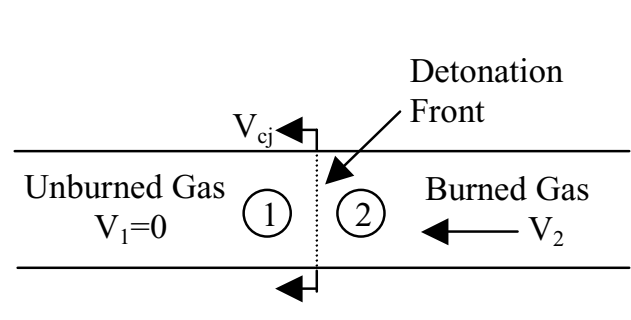

Stationary Reference Frame (SPARK)

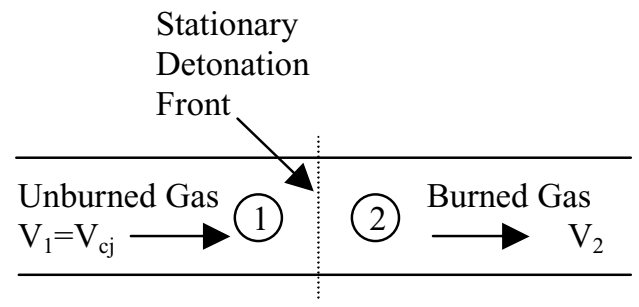

Reference Frame Fixed to Detonation Wave (ZND)

Figure 15 - Stationary and Detonation Wave Reference Frames

Figures 16 through 22 show the values of pressure, temperature, velocity, and $\mathrm{H}_{2} \mathrm{O}, \mathrm{OH}, \mathrm{O}$, and $\mathrm{H}$ mass fractions calculated by SPARK against those calculated by ZND using both the 7-step kinetic mechanism and the full 18-step mechanism. The 18step ZND results are shown to additionally quantify the accuracy of the 7-step approximation beyond what is shown in the Appendix. The results from ZND begin after the leading shock. The results shown for SPARK are taken from the baseline case, Case 1, which will be discussed further in Section 5. As shown, the ZND and SPARK results agree very well. The slight delay in completing the heat release that was discussed in the previous section is again evident in the temperature and $\mathrm{H}_{2} \mathrm{O}$ mass fraction plots. This is a very minor concern. Based on these results and those preceding, it appears that SPARK with the 7 -step $\mathrm{H}_{2}$-air mechanism is adequate for the desired detonation calculations. 


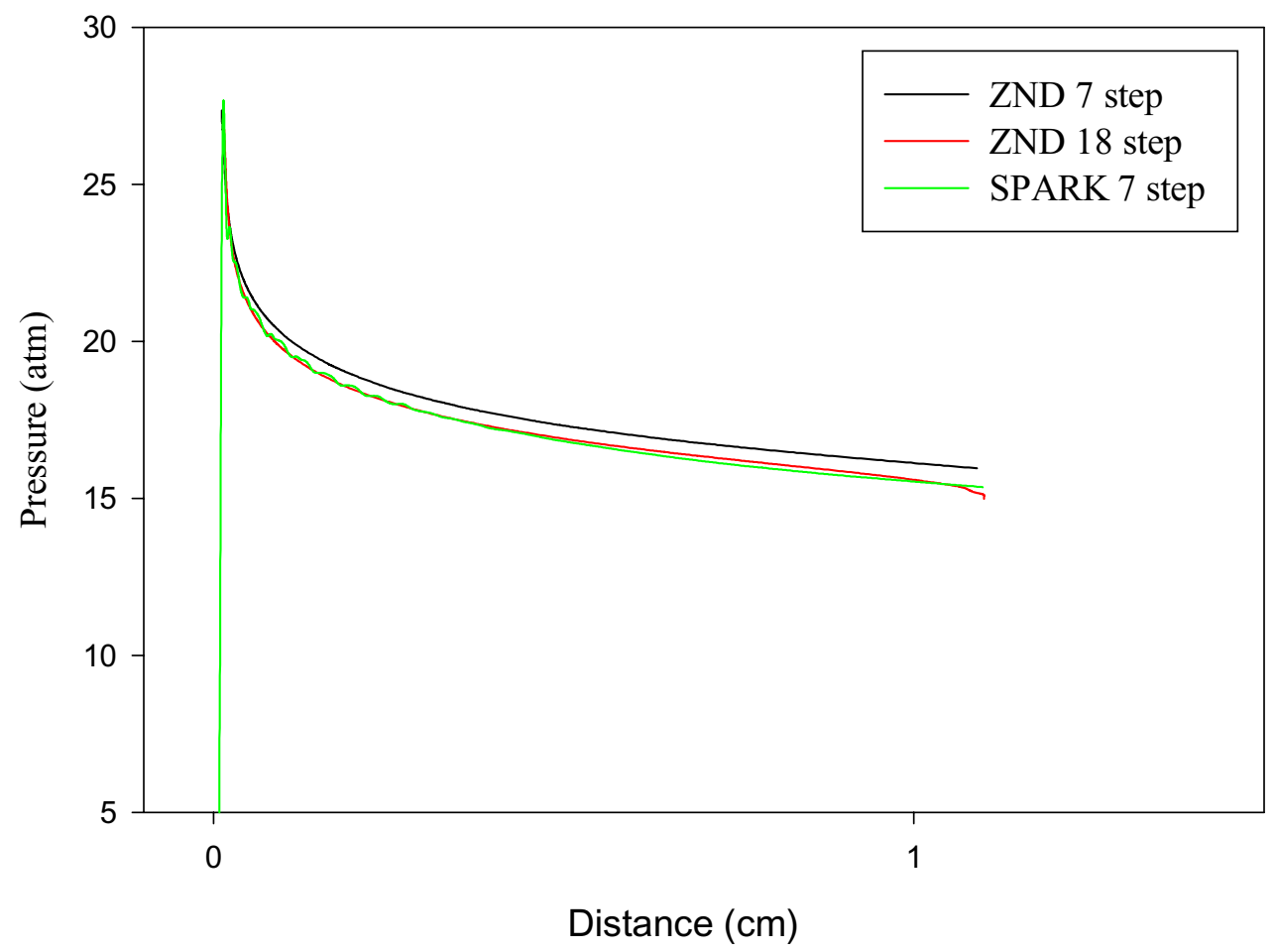

Figure 16 - Comparison of Pressure Profiles Between SPARK and ZND Codes

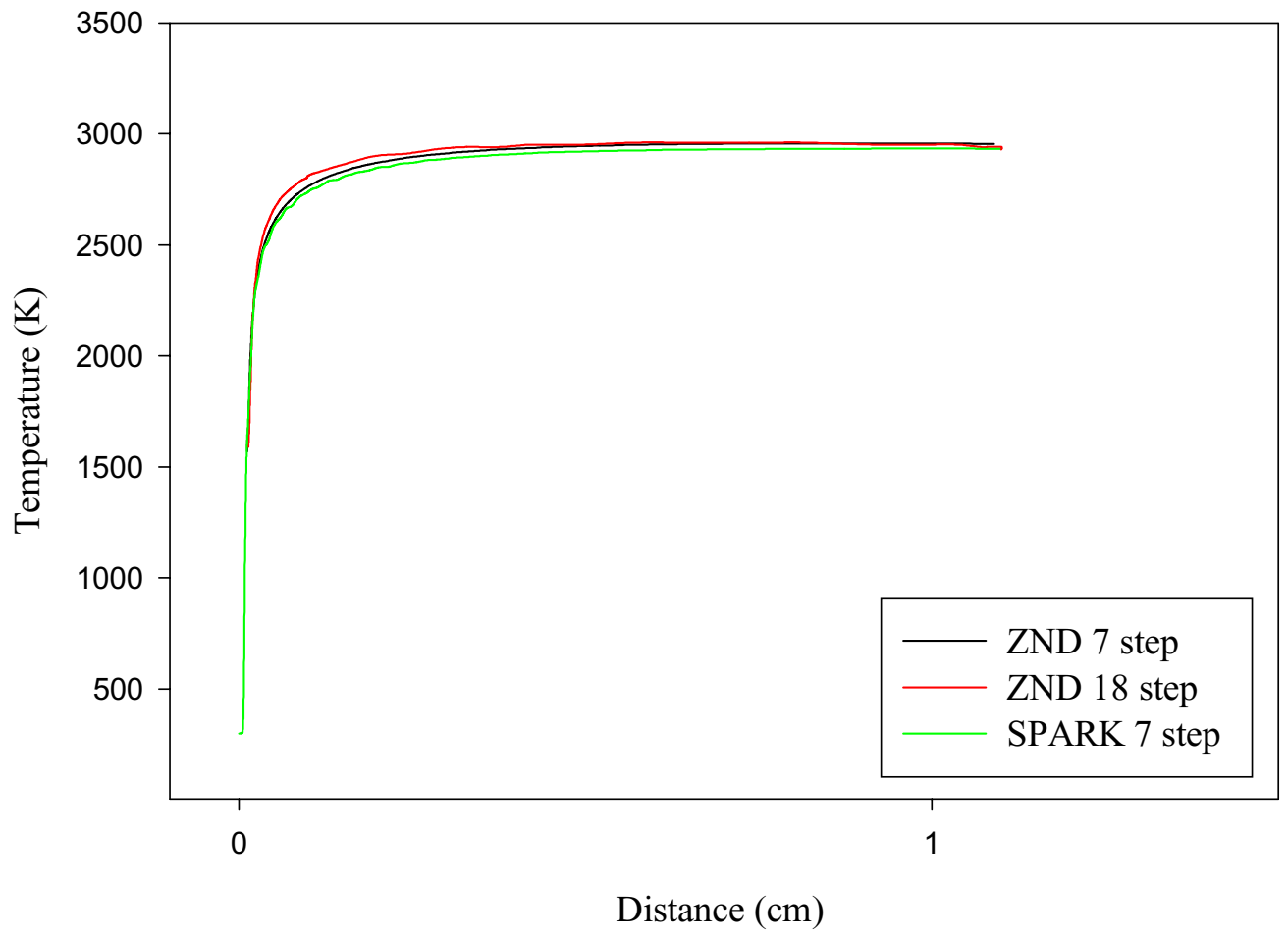

Figure 17 - Comparison of Temperature Profiles Between SPARK and ZND Codes 


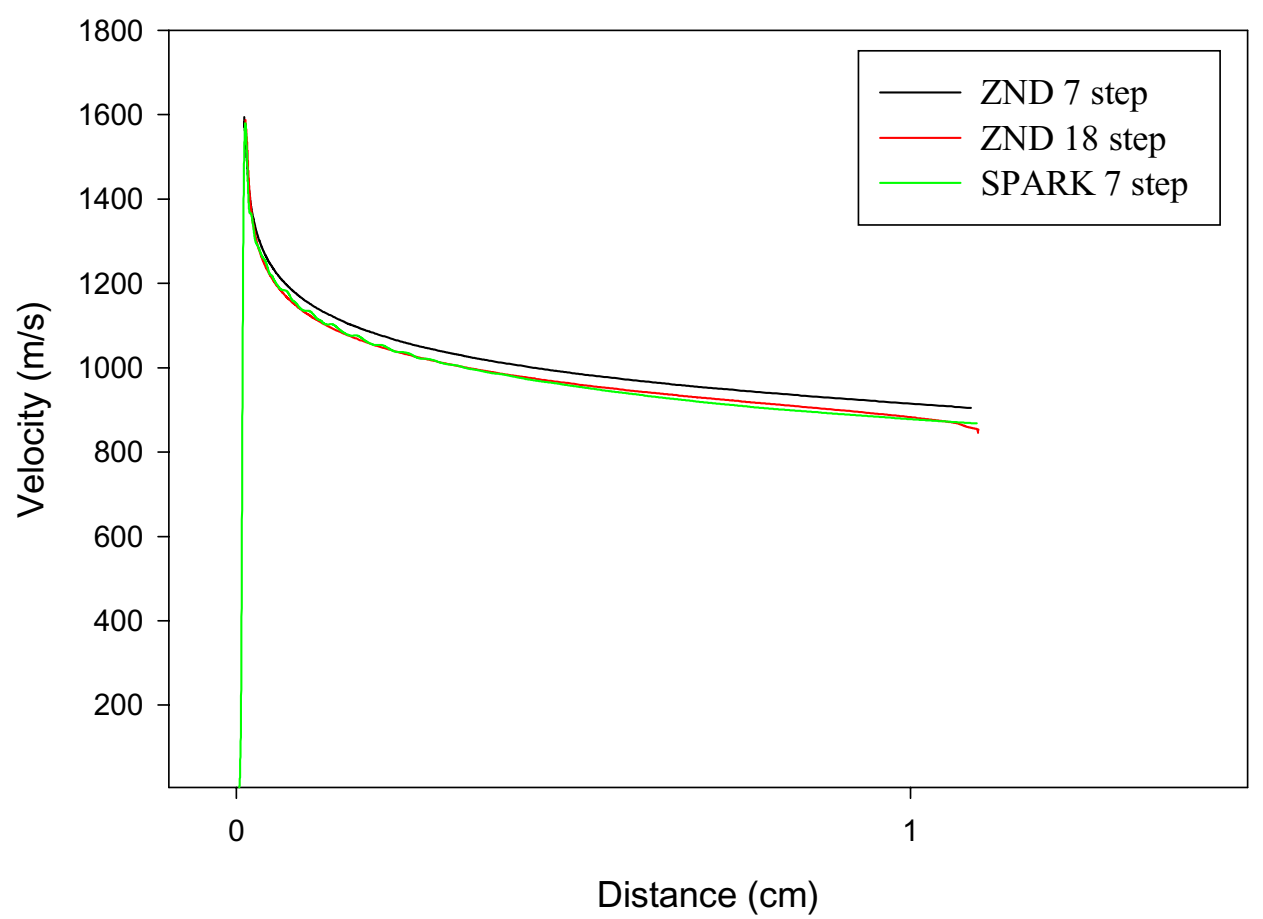

Figure 18 - Comparison of Velocity Profiles Between SPARK and ZND Codes

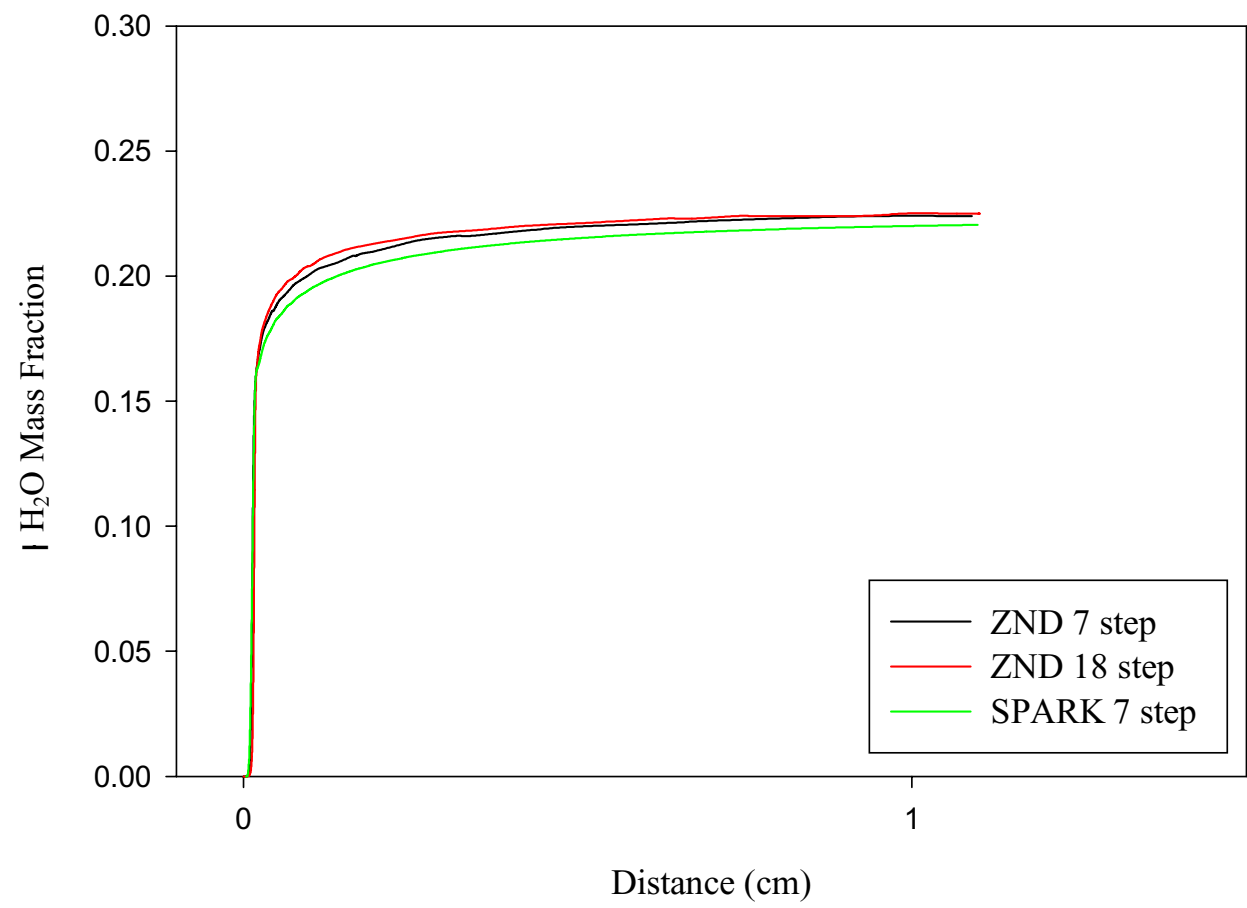

Figure 19 - Comparison of $\mathrm{H}_{2} \mathrm{O}$ Mass Fraction Profiles Between SPARK and ZND Codes 


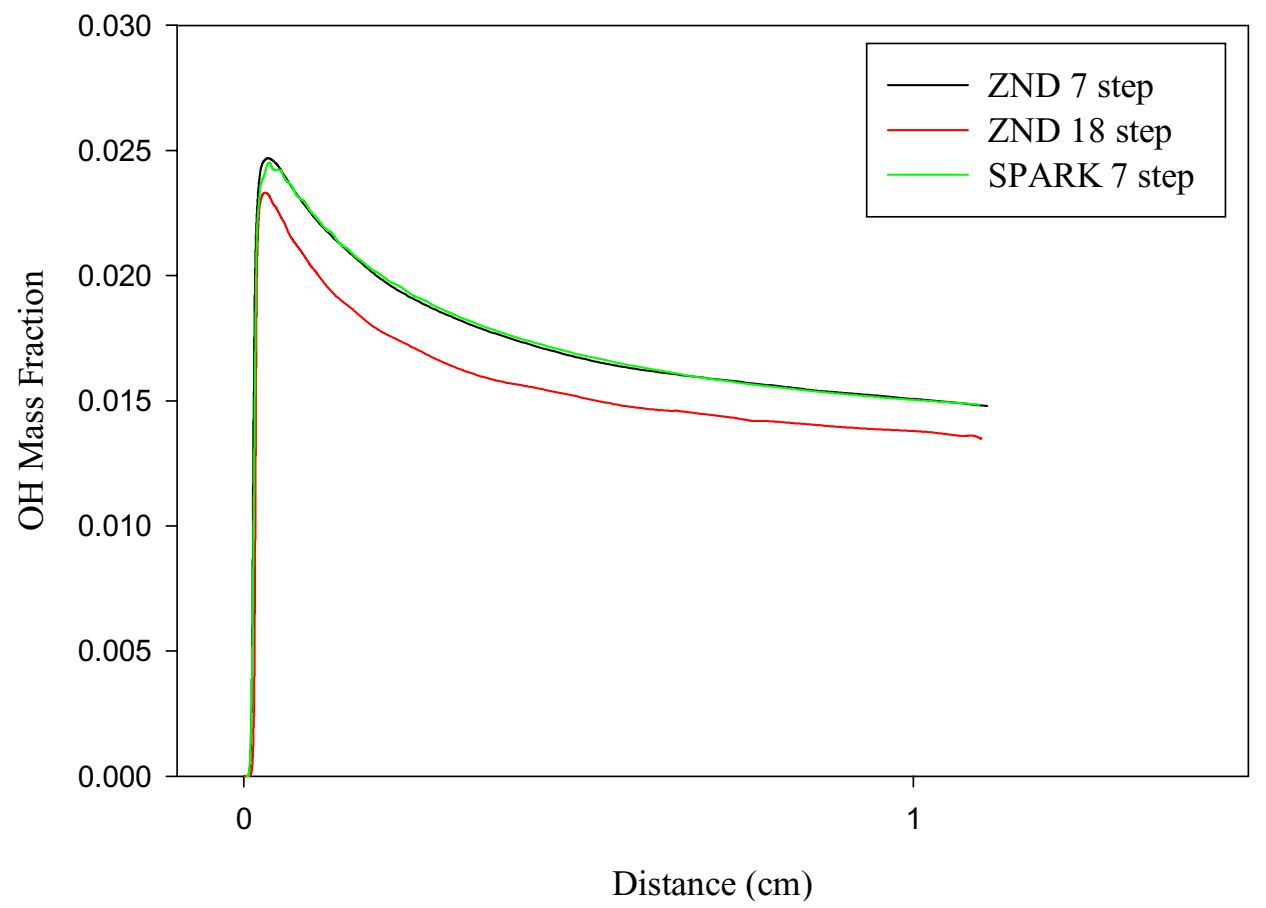

Figure 20 - Comparison of Detonation OH Mass Fraction Profiles Between SPARK and ZND Codes

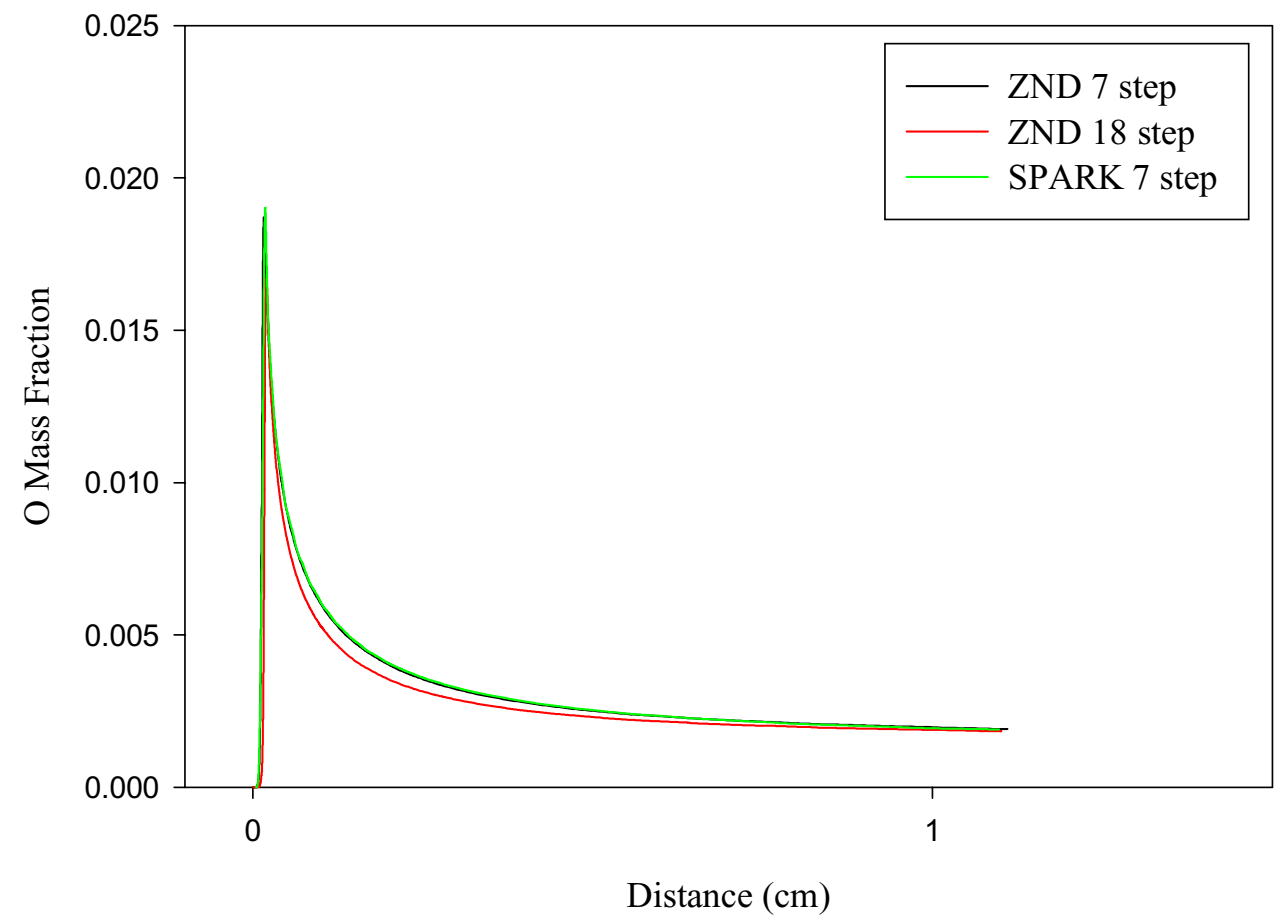

Figure 21 - Comparison of Detonation O Mass Fraction Profiles Between SPARK and ZND Codes 


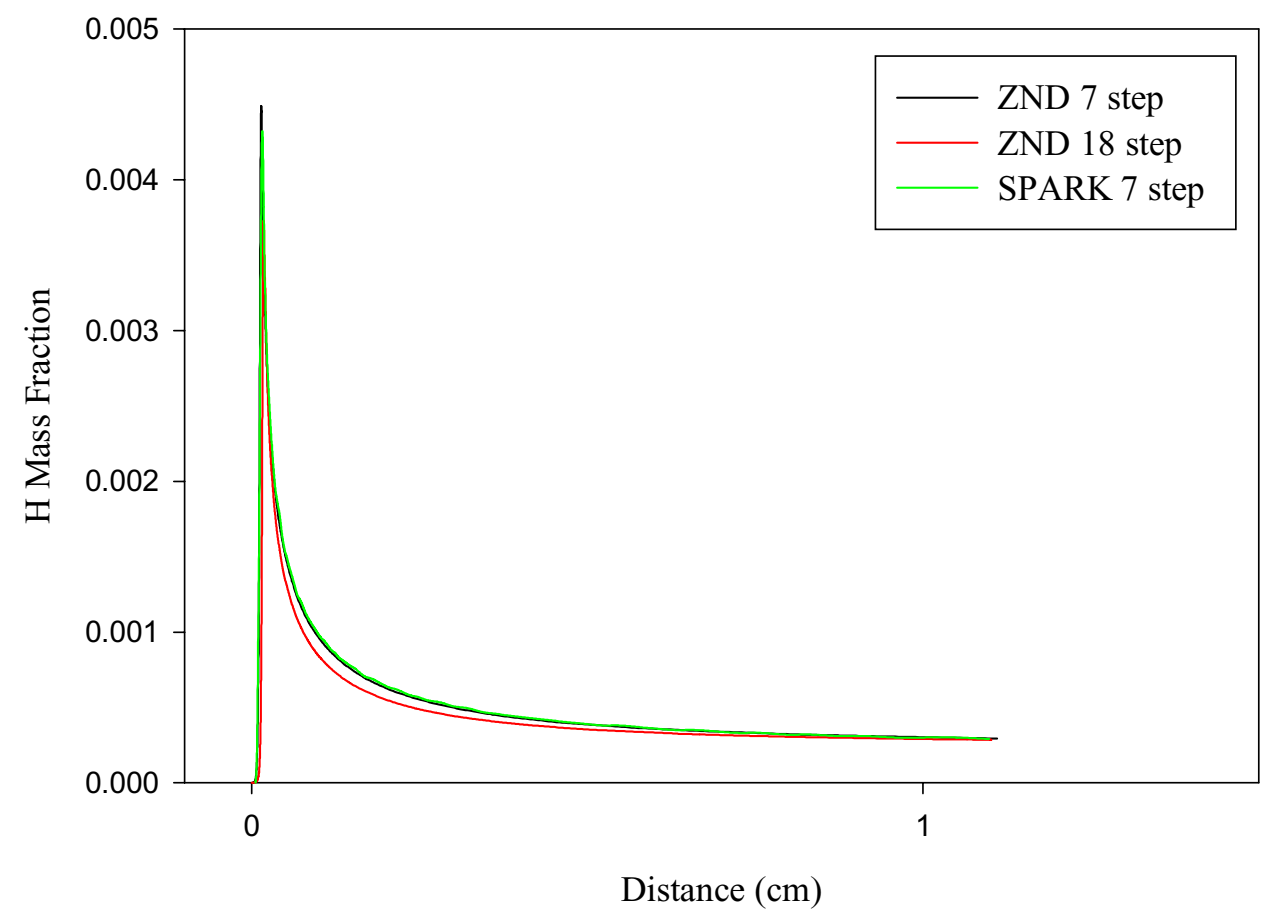

Figure 22 - Comparison of Detonation H Mass Fraction Profiles Between SPARK and ZND Codes

\section{Computational Results}

Having shown the general applicability of the SPARK code with the 7-step $\mathrm{H}_{2} /$ air mechanism for detonation calculations, we will now look at the results of each of the 11 cases outlined in Section 2.5. Figure 4 is repeated below as Figure 23 as a reminder of the geometry and detonation initiation/stabilization approach used throughout the study.

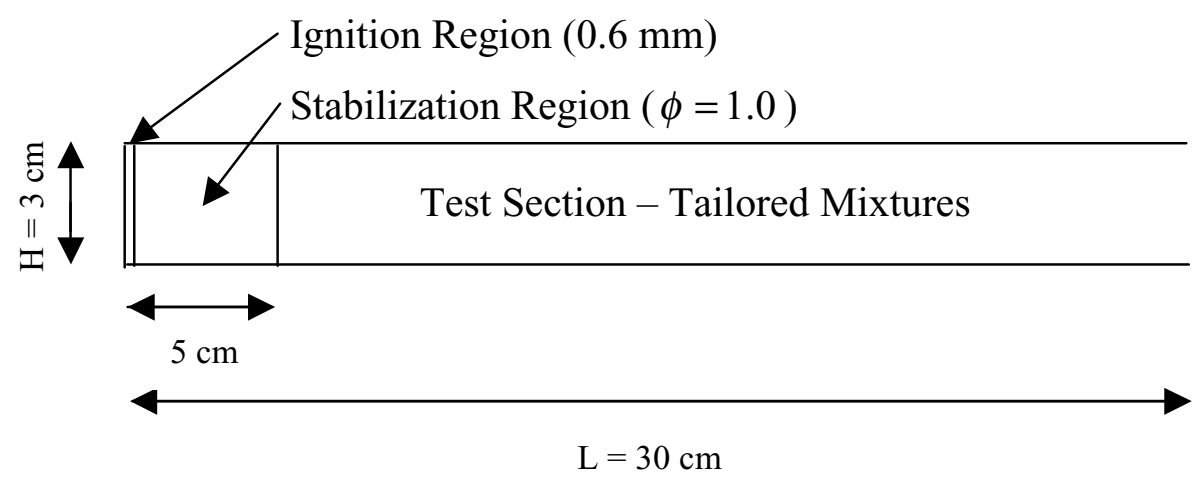

Figure 23 - Geometry for Numerical Study 


\subsection{Introduction}

Before moving on to compare the results of the subject test cases, there are a number of introductory questions that need to be addressed. These questions deal with the stability of both the numerical solution and the detonation itself, the appropriate impulse calculation to use in the $\mathrm{I}_{\mathrm{sp}}$ calculation, and the effect of equivalence ratio on $\mathrm{I}_{\mathrm{sp}}$ for uniform mixtures. It will also be useful to look at simplifications that can be made in the analysis of the 2-dimensional numerical results. These issues can all be addressed by looking at elements of the solutions that will be more fully presented in later sections.

\subsubsection{Dimensionality of Results}

It is appropriate to focus on the last issue first, namely, any simplifications that can be made in analyzing the 2-dimensional results obtained from SPARK. Detonation tubes are often simulated using 1-dimensional codes, as demonstrated by Kailasanath and Patniak (1999), Wilson and Paxson (2001), and Sterling et al. (1995). If the assumption of primarily 1-dimensional behavior for uniform and axially varying mixtures can be verified, then those cases can be visualized and compared by looking at 1-dimensional slices along the length of the tube, typically taken along the centerline. It is obvious that the transversely varying mixtures will still need to be examined to some extent using 2dimensional methods, but even these may be looked at 1-dimensionally for some flow variables.

Figures 24 to 28 show 2-dimensional plots of pressure, transverse velocity and temperature from Cases 1, 2, 3, 4, and 7 as examples of a completely uniform mixture, a transversely varying mixture, an axially varying mixture, a uniform mixture with a step change in equivalence ratio along the tube, and a partially filled tube, respectively. All the plots in Figures 24 to 28 represent the flowfield just prior to the detonation wave exiting the tube. While all the cases show small traces of transverse velocity, only Case 2 shows any signs of significant multi-dimensionality, seen in the temperature plot for that case. However, the pressure plot for Case 2 is still very 1-dimensional, which is to be expected, since there can be no net flow in the transverse direction. Thus for all the cases, one-dimensional comparisons of pressure should be valid.

It is of interest that there is no observed boundary layer in any of the plots. A boundary layer is present in all cases, but it is so thin as to be unobservable in the scale of the plots, and it has essentially no effect on the flow features of the cases under study. It should also be noted that in Case 7, a partial fill case, the detonation wave has propagated into the air only region at the end of the tube, leading to the region of lower temperature at the end of the tube seen in that plot. 
3098310

2498839

1899368

1299897

700426

100955

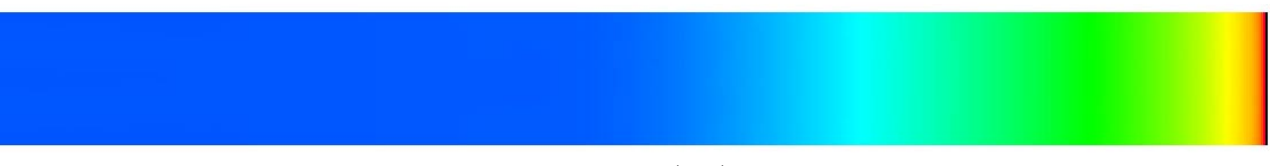

Pressure $(\mathrm{Pa})$
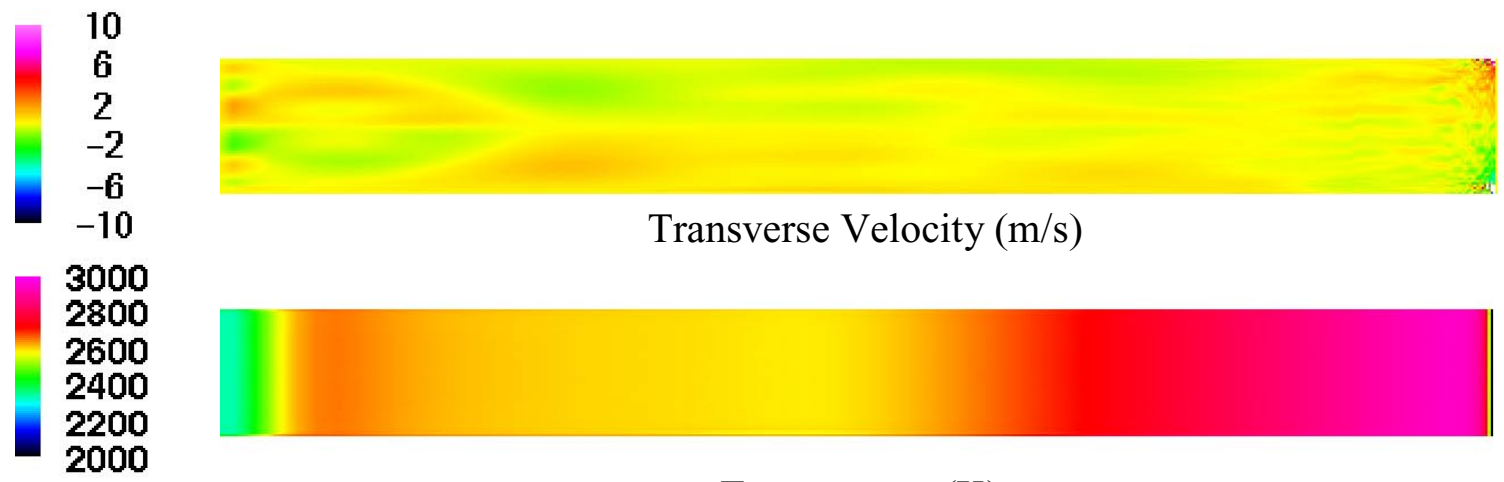

Temperature (K)

Figure 24 - Case 1 Pressure, Transverse Velocity, and Temperature Contours

320222
258198
196175
1341514
721279
101044
10
6
2
-2
-6
-10

3000
2800
2600
2400
2200
2000

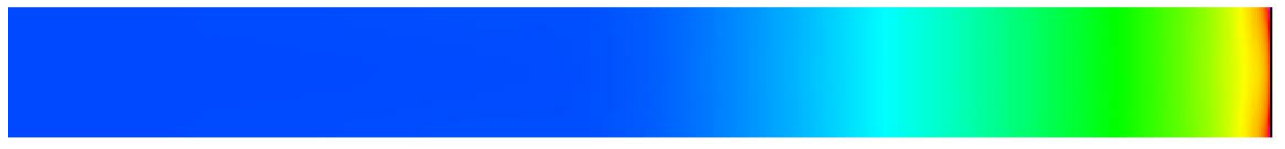

Pressure $(\mathrm{Pa})$

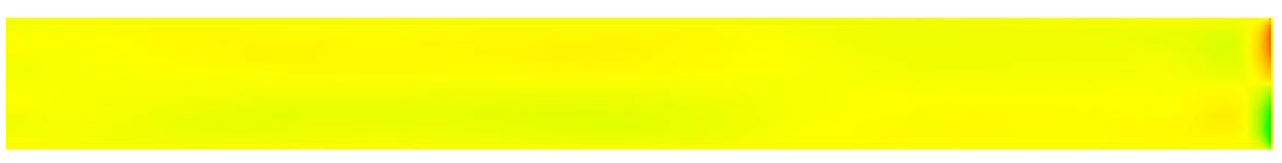

Transverse Velocity $(\mathrm{m} / \mathrm{s})$

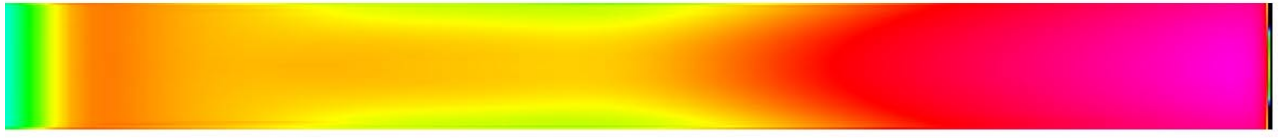

Temperature $(\mathrm{K})$

Figure 25 - Case 2 Pressure, Transverse Velocity, and Temperature Contours 
3113750
2511250
1908749
1306248
703748

101247

10

6

2

$-2$

$-6$

$-10$

3000

2800

2600

2400

2200

2000

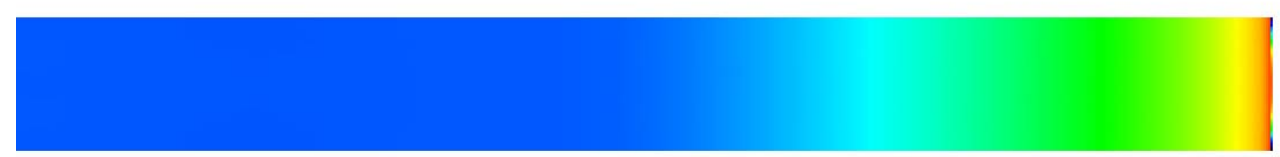

Pressure $(\mathrm{Pa})$

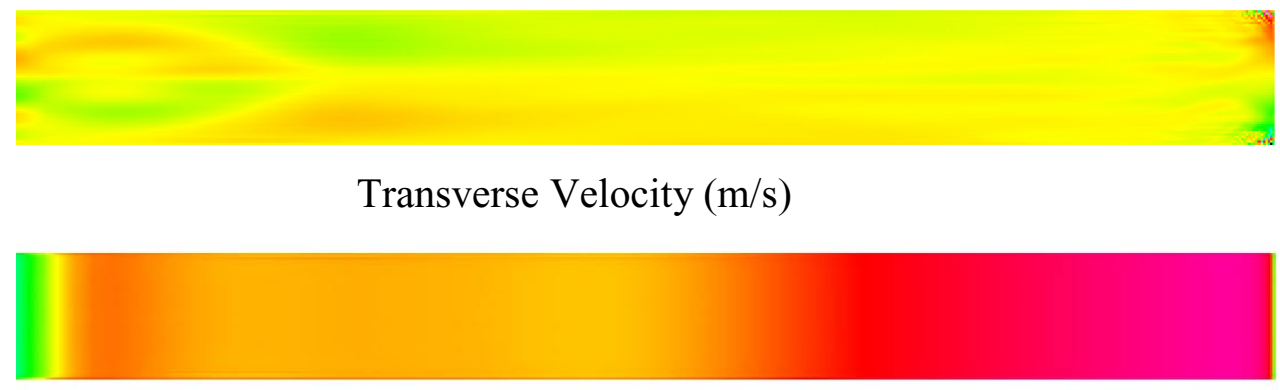

Temperature $(\mathrm{K})$

Figure 26 - Case 3 Pressure, Transverse Velocity, and Temperature Contours

3113750
2511250
1908749
1306248
703748
101247
10
6
2
-2
-6
-10
3000
2800
2600
2400
2200
2000

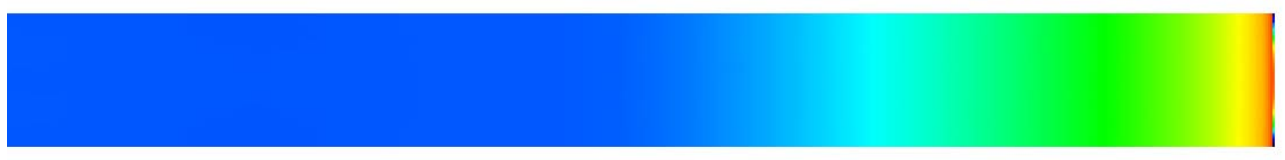

Pressure $(\mathrm{Pa})$

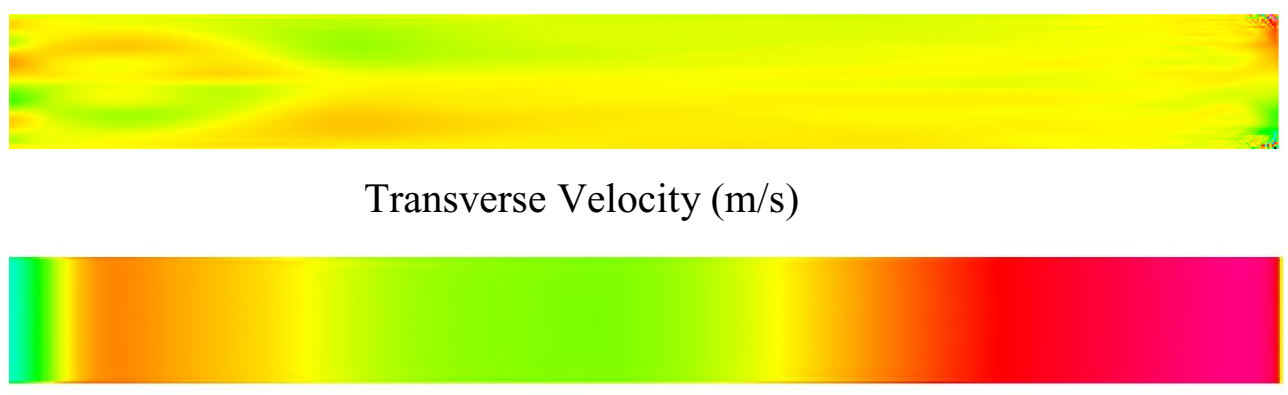

Temperature $(\mathrm{K})$

Figure 27 - Case 4 Pressure, Transverse Velocity, and Temperature Contours 


\section{0 \\ 1571298 \\ 1203786 \\ 836273 \\ 468761 \\ 101249}

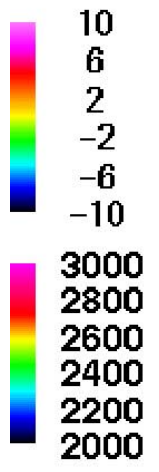

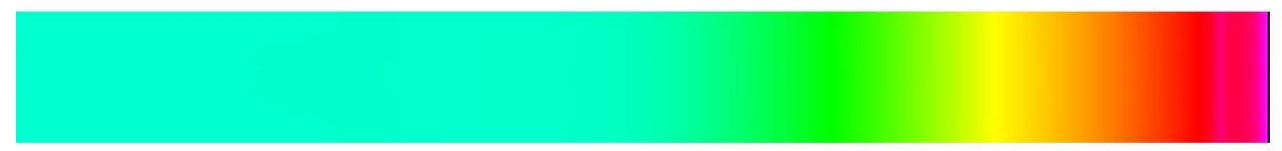

Pressure $(\mathrm{Pa})$

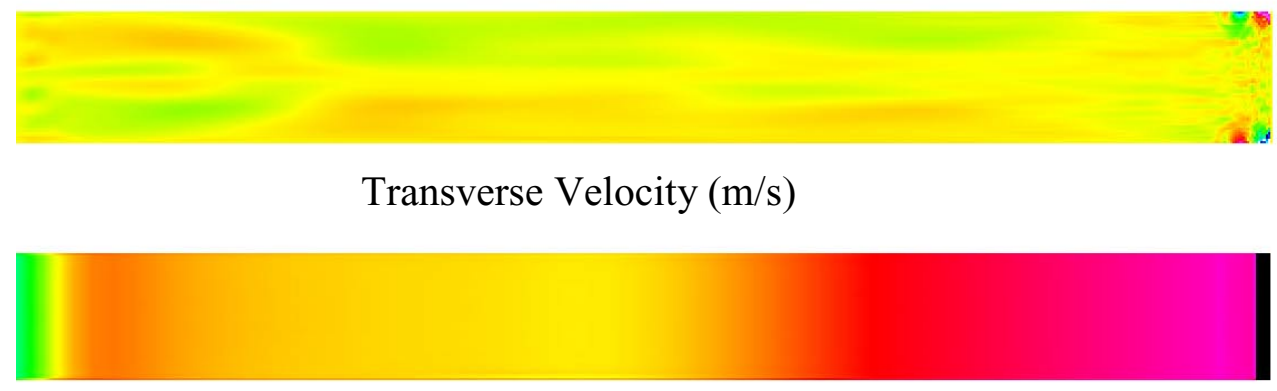

Temperature $(\mathrm{K})$

Figure 28 - Case 7 Pressure, Transverse Velocity, and Temperature Contours

\subsubsection{Numerical and Detonation Stability}

It is of great importance to establish that each simulation is both numerically and physically stable. This is most easily accomplished by looking at the detonation wave speed. The wave speed was determined by tracking the wave position, defined as the tube centerline position with the steepest X-velocity gradient, as a function of time during the wave propagation period of the calculation. The wave speed was found to be very sensitive to both concerns during the validation stages of this study. The equilibrium value of the Chapman-Jouget wave speed is easily calculable using the CEA code of Gordon and Mcbride (1994). In Figure 29, the three baseline case wave speeds are compared to each other, from which it can be seen that each simulation is stable, and that wave speed is proportional to equivalence ratio. Figures 30 through 33 show the wave speed for each baseline case compared to several reference detonation wave speeds calculated using the CEA code. After being initially overdriven to insure detonation initiation, the wave relaxes to just under the CJ wave speed in each case, and then slowly converges on the equilibrium CJ wave speed throughout the "steady state" propagation. The steady state wave speed is generally within $2 \%$ to $3 \%$ of the equilibrium value, also indicative of a stable detonation. 


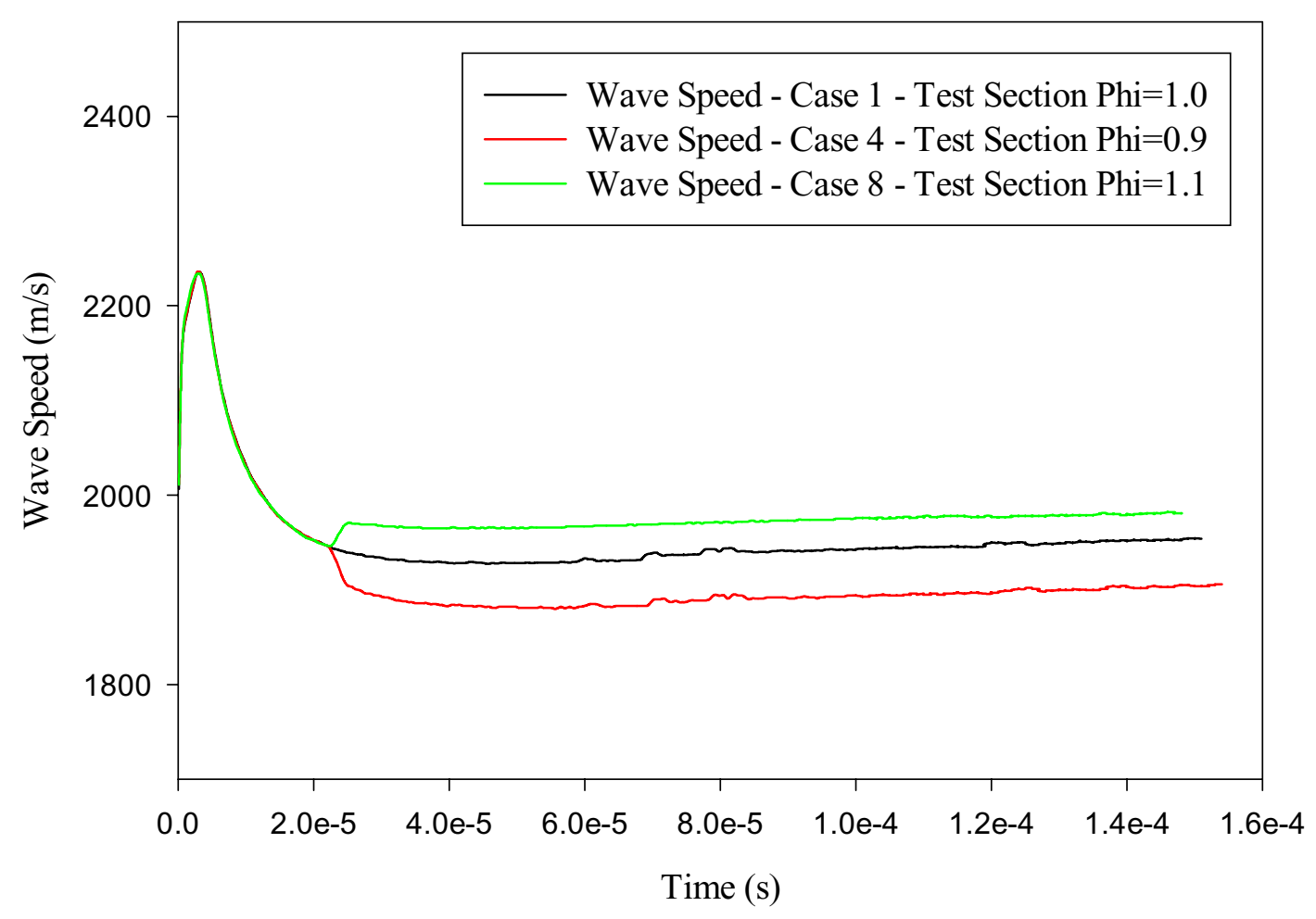

Figure 29 - Detonation Wave Speeds for Cases 1, 4, and 8

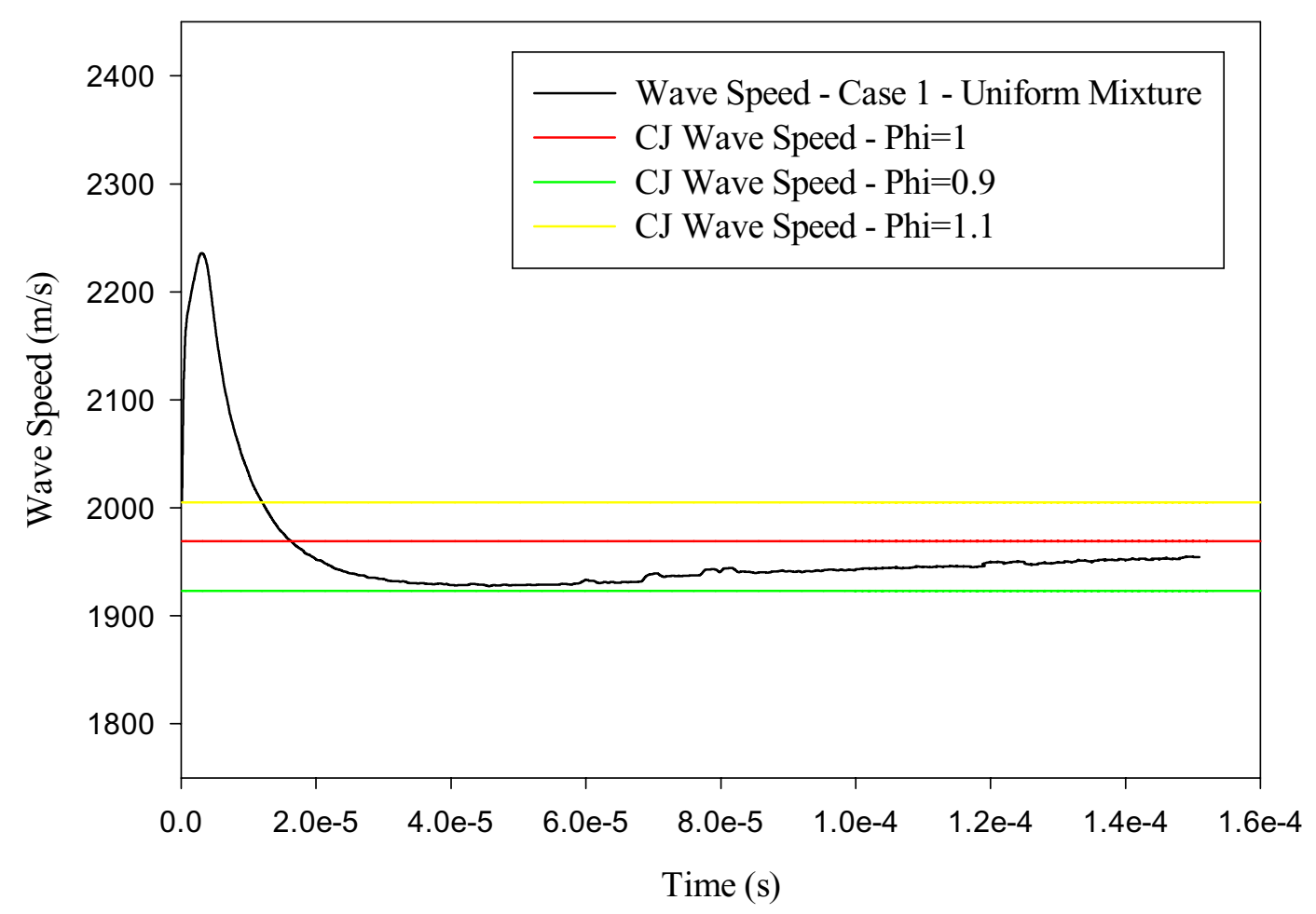

Figure 30 - Case 1 ( $\Phi=1.0$ ) Wave Speed Compared to Equilibrium CJ Wave Speeds 


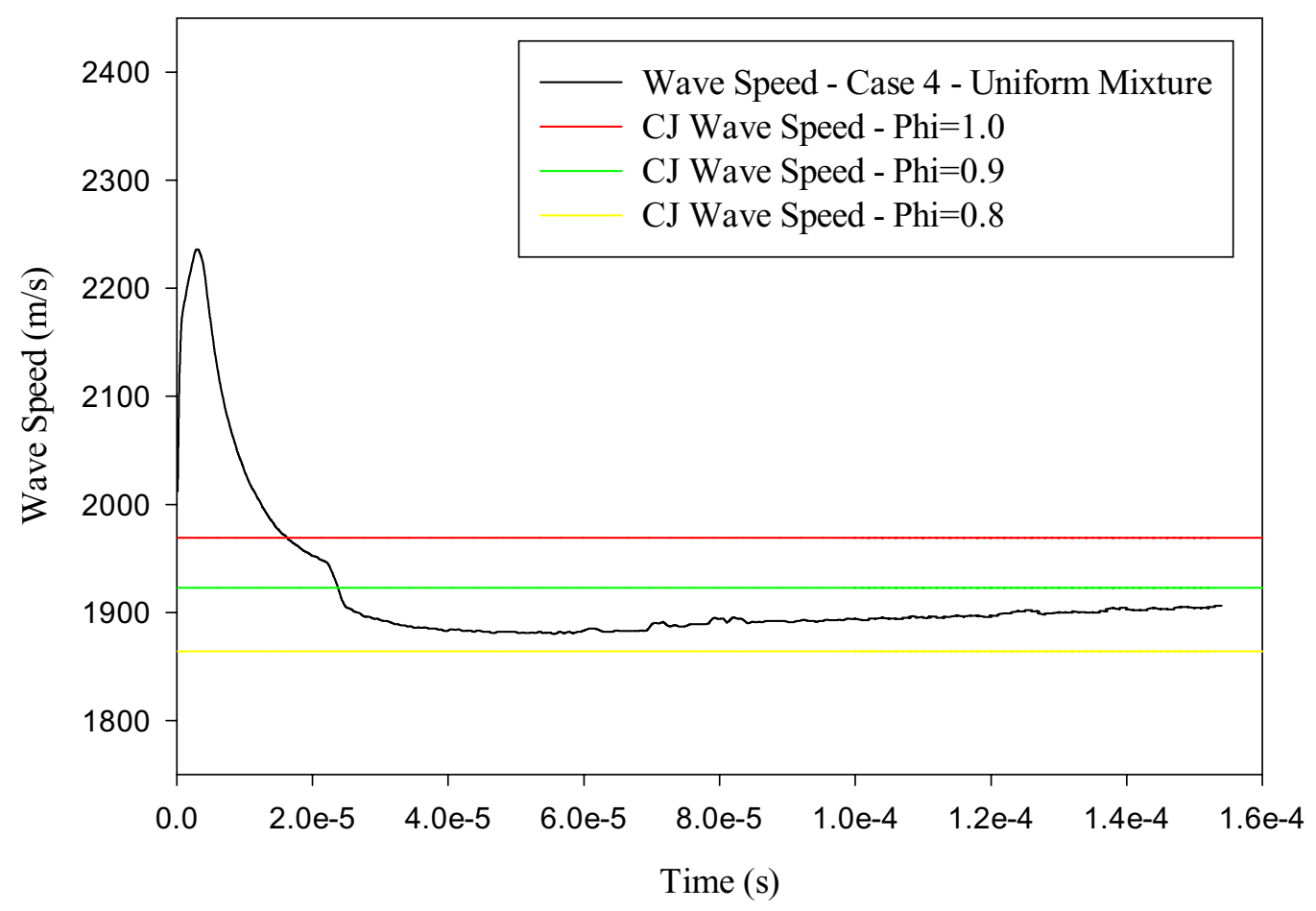

Figure 31 - Case 4 ( $\Phi=0.9$ ) Wave Speed Compared to Equilibrium CJ Wave Speeds

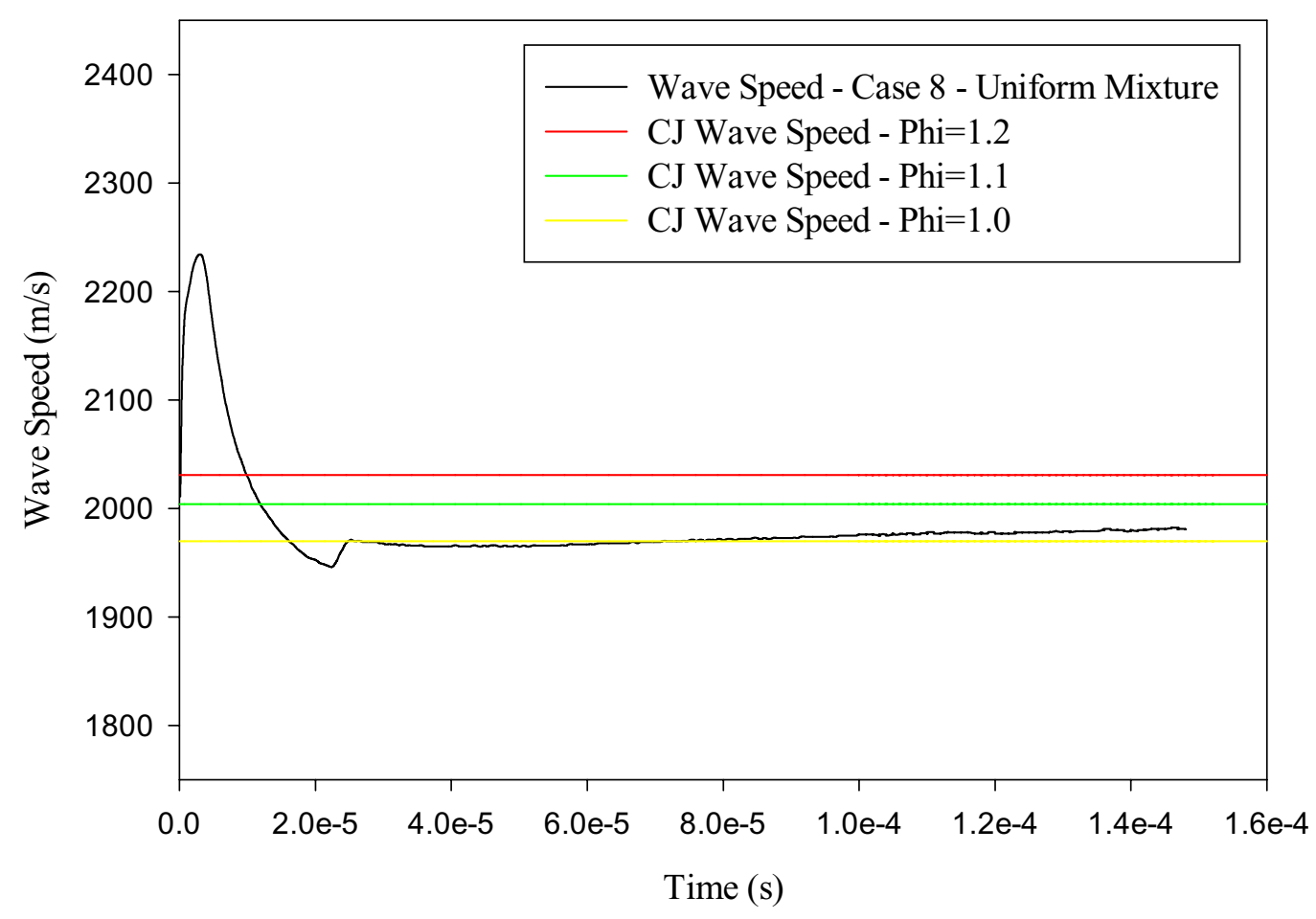

Figure 32 - Case 8 ( $\Phi=1.1)$ Wave Speed Compared to Equilibrium CJ Wave Speeds 


\subsubsection{Total Impulse Calculation}

As discussed in Section 2.4, there are two methods for calculating the impulse generated by the detonation and subsequent blowdown. The first is to integrate the pressure acting on the head-end of the tube over the cycle time, subtracting off the viscous drag of the walls, as given in equation 17. It is also acceptable to integrate the momentum flux exiting the tube over the cycle time, correcting for the incomplete expansion to ambient pressure, as given in equation 18. Figure 33 shows the results of both impulse calculations versus time for Case 1. It is not possible to differentiate the impulse generated by the initiation and stabilization zones, so calculated total impulse includes the effects of these regions. The weight of $\mathrm{H}_{2}$ in the stabilization zone is included in the total fuel weight in order to calculate the overall specific impulse.

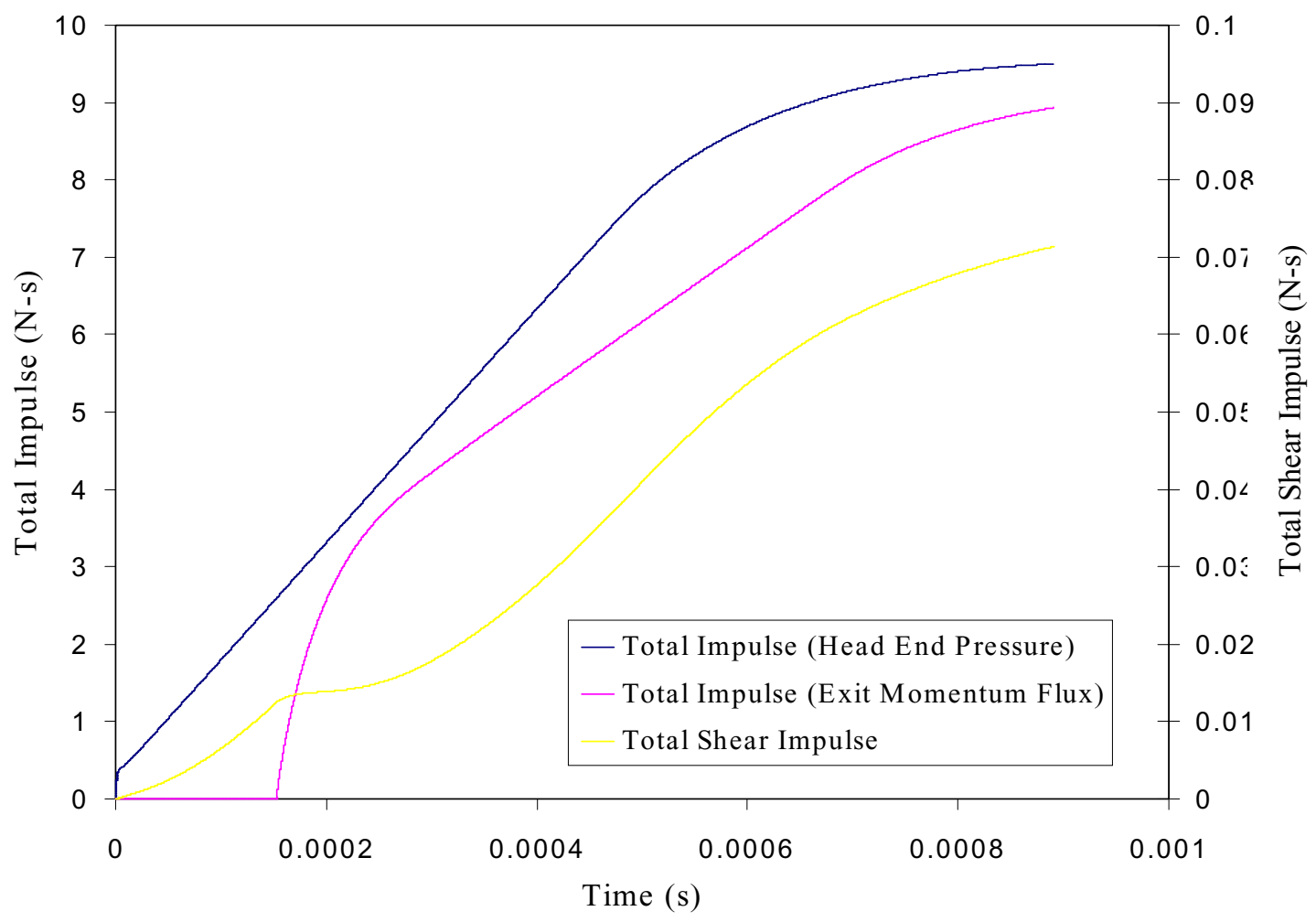

Figure 33 - Impulse Calculations for Case 1 - Uniform Stoichiometric Mixture

From Figure 33 it is seen that the head end pressure calculation has come close to its maximum value, while the exit momentum flux calculation is still rising. The two impulse values are about $6 \%$ apart at the termination of the calculation due to flow reversal at the tube exit boundary. This result is understandable in that flow is still exiting the tube at a considerable velocity at this point (see Figure 5). However, the pressure at the head-end has returned close to the initial pressure. The head-end pressure will in fact go below the ambient pressure if the calculation is allowed to continue due to reflected expansion waves in the tube. It is expected that if the calculation were run out far enough so that the tube returned to a quiescent state, these two impulse values would 
equalize. However, the point at which the head-end pressure begins to go subatmospheric is precisely the point at which it would be expected that the inlet air valves would open, drawing in fresh fuel and air and preventing the head pressure from going significantly negative. Since the thrust wall pressure integration has approached a maximum, it is evident that this is the value to use in the $\mathrm{I}_{\mathrm{sp}}$ calculation, being the closest to the expected maximum value. All further references to $I_{\mathrm{sp}}$ values are based on the head-end pressure integration method of equation 17.

Before leaving Figure 33, it is interesting to note that the wall shear force impulse penalty also included with the figure shows that the wall drag only represents a penalty of approximately $0.75 \%$ of the total impulse. While the viscous drag is included in the total impulse calculation, it could be readily ignored without significantly altering the results of any of the study cases.

\subsubsection{Comparison of Baseline Uniformly Fueled Cases}

It will be useful in understanding the effect of equivalence ratio gradients if the general effect of equivalence ratio in uniformly fueled mixtures in the range of interest is known. Figure 34 shows that $\mathrm{I}_{\mathrm{sp}}$ is inversely proportional to equivalence ratio, with the lean case achieving the highest $\mathrm{I}_{\mathrm{sp}}(4706 \mathrm{~s})$, the stoichiometric case in the middle (4450 $\mathrm{s})$, and the fuel rich case yielding the lowest $\mathrm{I}_{\mathrm{sp}}(4177 \mathrm{~s})$. If these maximum $\mathrm{I}_{\mathrm{sp}}$ values are plotted against overall equivalence ratio, as in Figure 35 , it is seen that the $I_{s p}$ dependence on equivalence ratio in this range is approximately linear. Inclusion of the stabilization zone raises the overall equivalence ratio of the fuel lean cases to 0.92 , and lowers the overall equivalence ratio of the fuel rich cases to 1.08. Since the effect of the stabilization zone cannot be isolated within the calculation, this equivalence ratio bias is included in all Isp calculations.

Since $I_{s p}$ is calculated from the force generated by the fluid pressure pushing against the closed end of the tube, the head-end pressure can be plotted against time to get more insight into the source of the variation in $\mathrm{I}_{\mathrm{sp}}$ with equivalence ratio, as in Figure 36. It is evident that decreasing the equivalence ratio below an equivalence ratio of one decreases the plateau pressure achieved, but not in proportion to the decrease in the amount of fuel used, thus causing an increase in $\mathrm{I}_{\text {sp }}$ for Case 4. However, no significant increase in plateau pressure was achieved by over-fueling the tube as in Case 8 , causing a decrease in $\mathrm{I}_{\mathrm{sp}}$ due to the increase in fuel used. It is of interest to note that the duration of the pressure plateau and the slope of the blowdown did not change significantly as a function of equivalence ratio. 


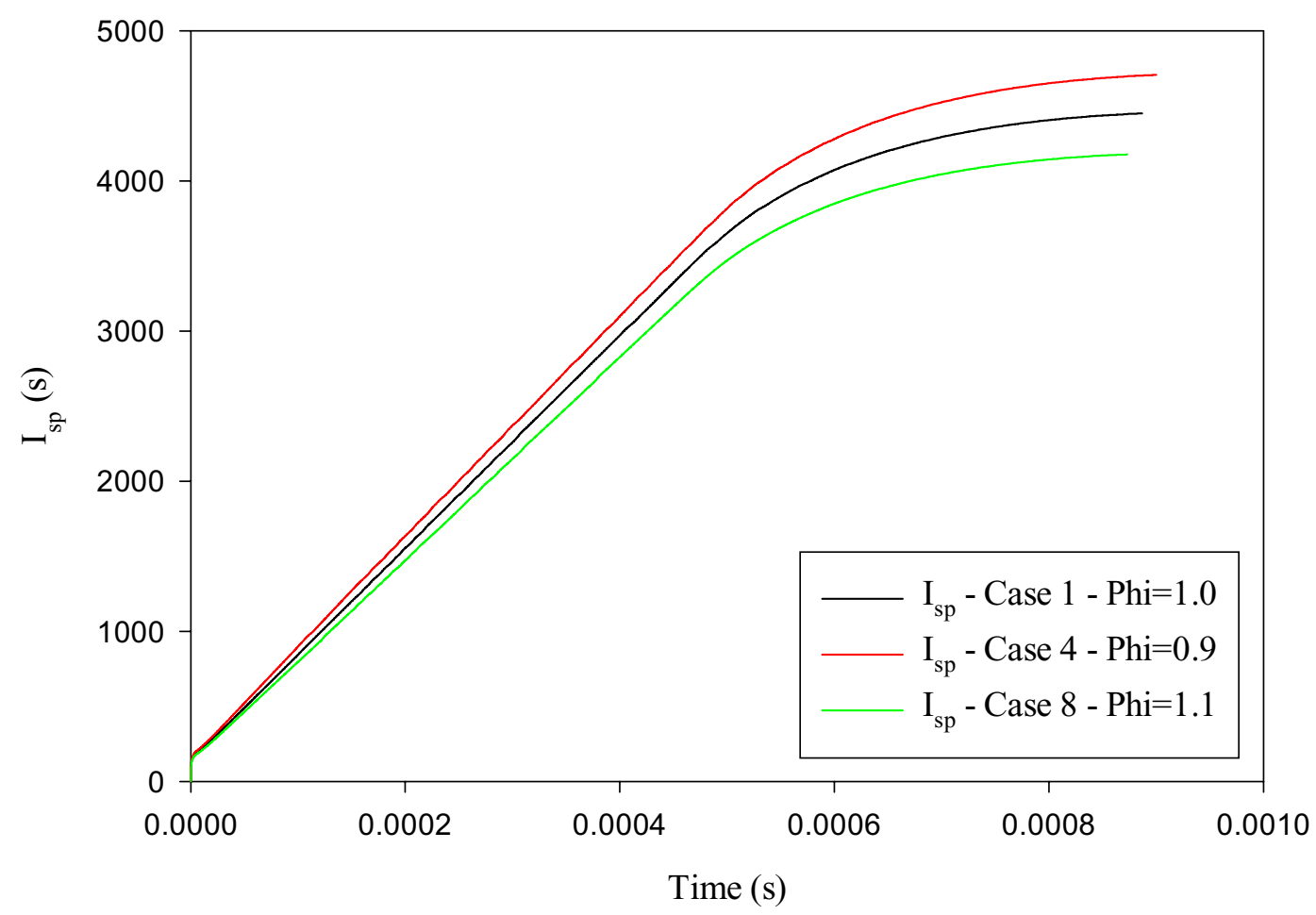

Figure $34-\mathrm{I}_{\mathrm{sp}}$ for Baseline Uniformly Fueled Cases 1, 4, and 8

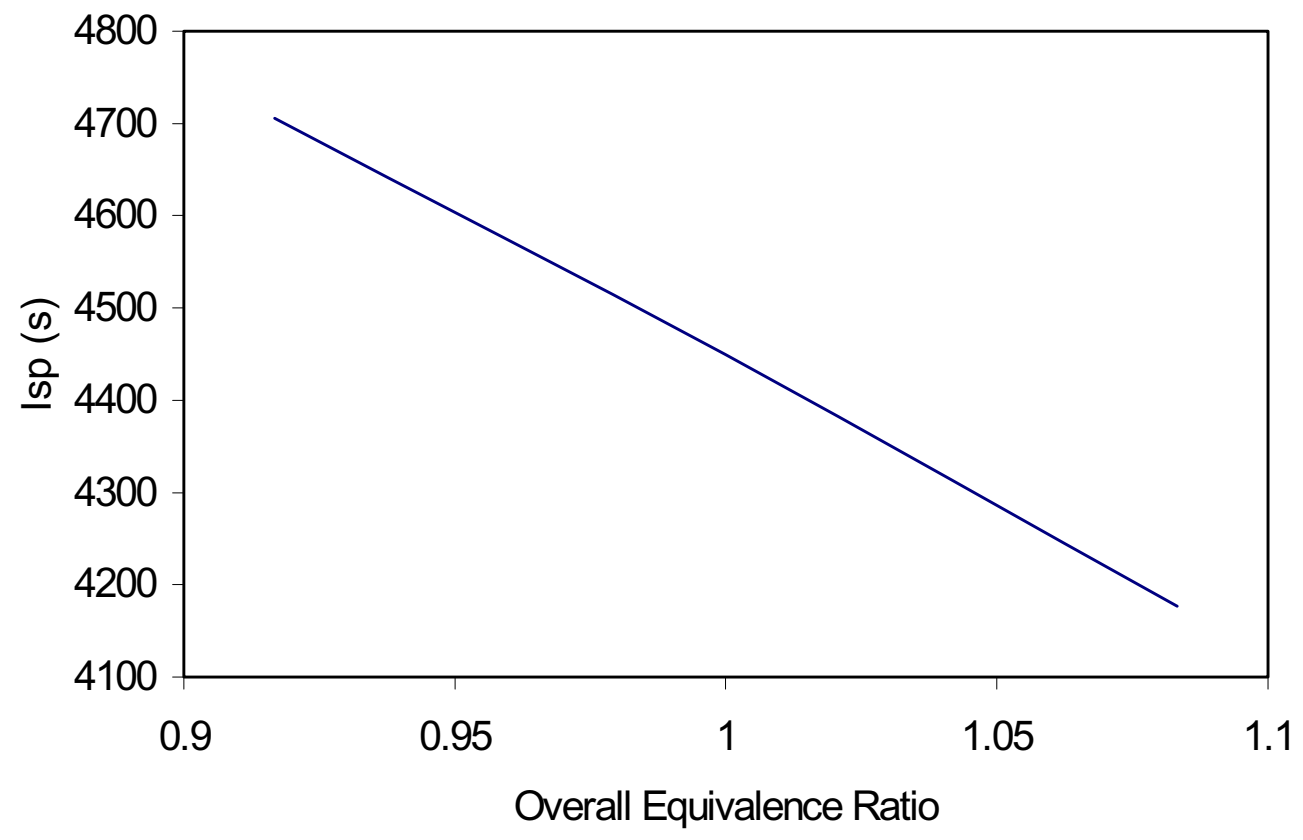

Figure $35-\mathrm{I}_{\mathrm{sp}}$ as a Function of Overall Equivalence Ratio, including Stabilization Zone, for Cases 1,4 , and 8 


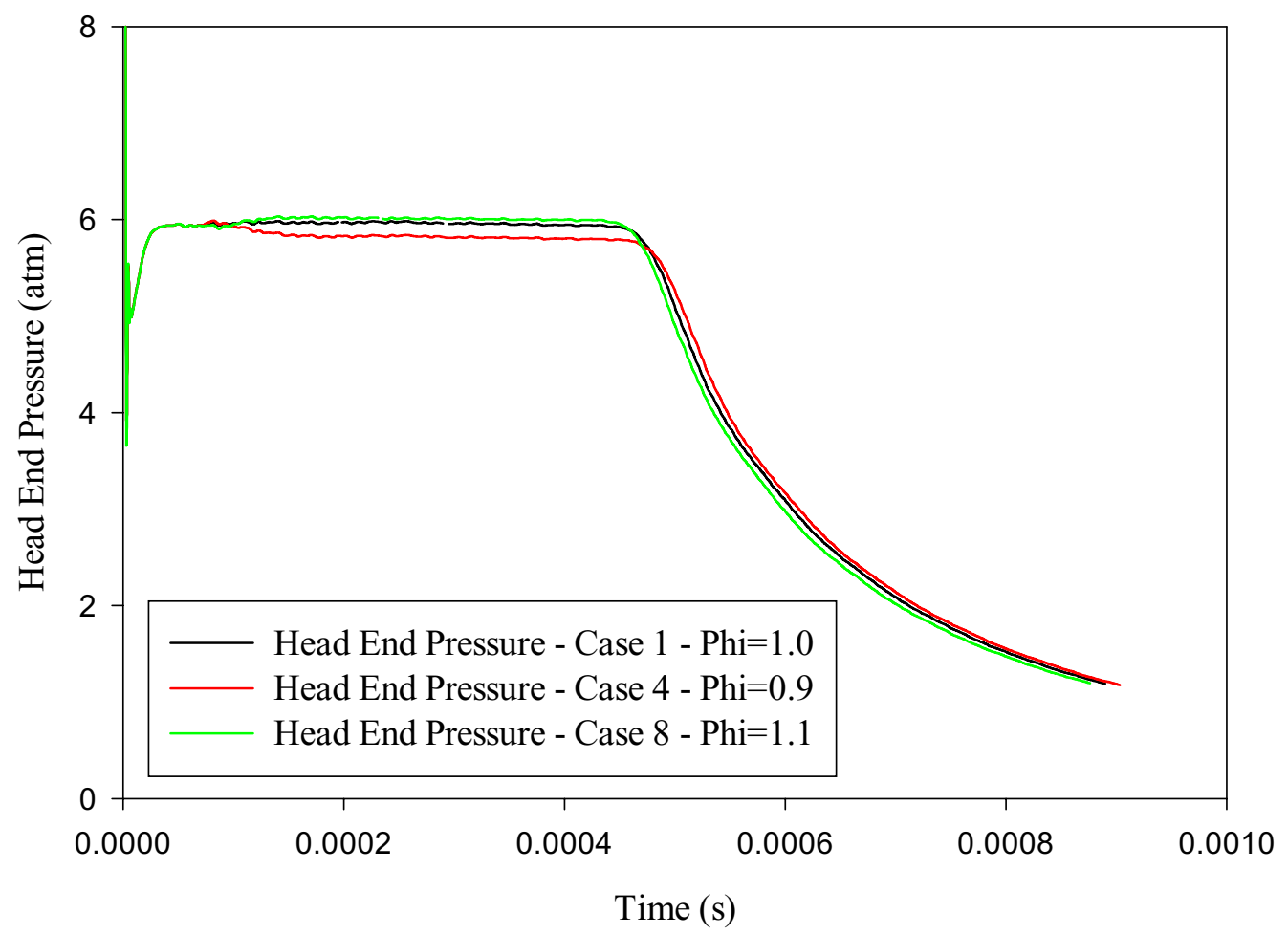

Figure 36 - Detonation Tube Head-End Pressure for Cases 1, 4, and 8

Having dealt with these introductory issues, we can now move on to the analysis of the performance results for each group of mixtures.

\subsection{Stoichiometric Mixtures}

Before looking at the performance results, it is of value to verify the numerical and physical stability of the detonations. Figures 37 through 40 show the wave speed results for the first 3 cases, first compared to each other and then to several reference equilibrium values calculated using the CEA code. Each simulation is clearly stable. It is of interest to note that the transverse fuel distribution, Case 2, reaches the same wave speed as the uniformly fueled mixture, Case 1, after initial regions of overshoot and undershoot, and that this wave speed is stable. This indicates that the detonation wave is underdriven in the rich mixture and overdriven in the lean mixture. We will come back to this point after looking at the performance results. The wave speed of the axially varying fuel distribution case follows the initial equivalence ratio distribution fairly well, deviating from the equilibrium value slightly more at the step change in equivalence ratio located at the $5 \mathrm{~cm}$ point $\left(\sim 2.2 \times 10^{-5} \mathrm{~s}\right)$ as there is not enough time for the detonation wave to react to the change in mixture at that point. 


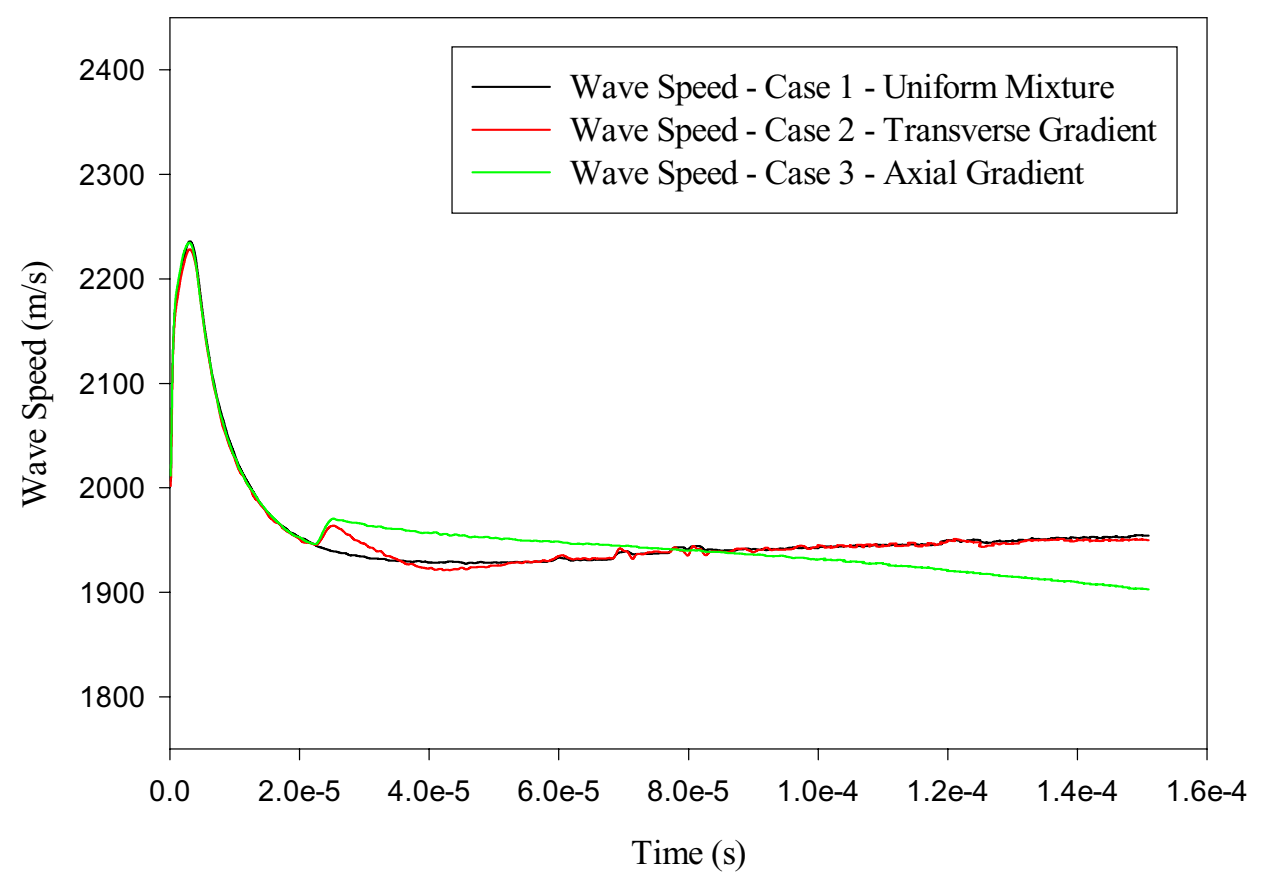

Figure 37 - Detonation Wave Speeds for Stoichiometric Cases 1, 2, and 3

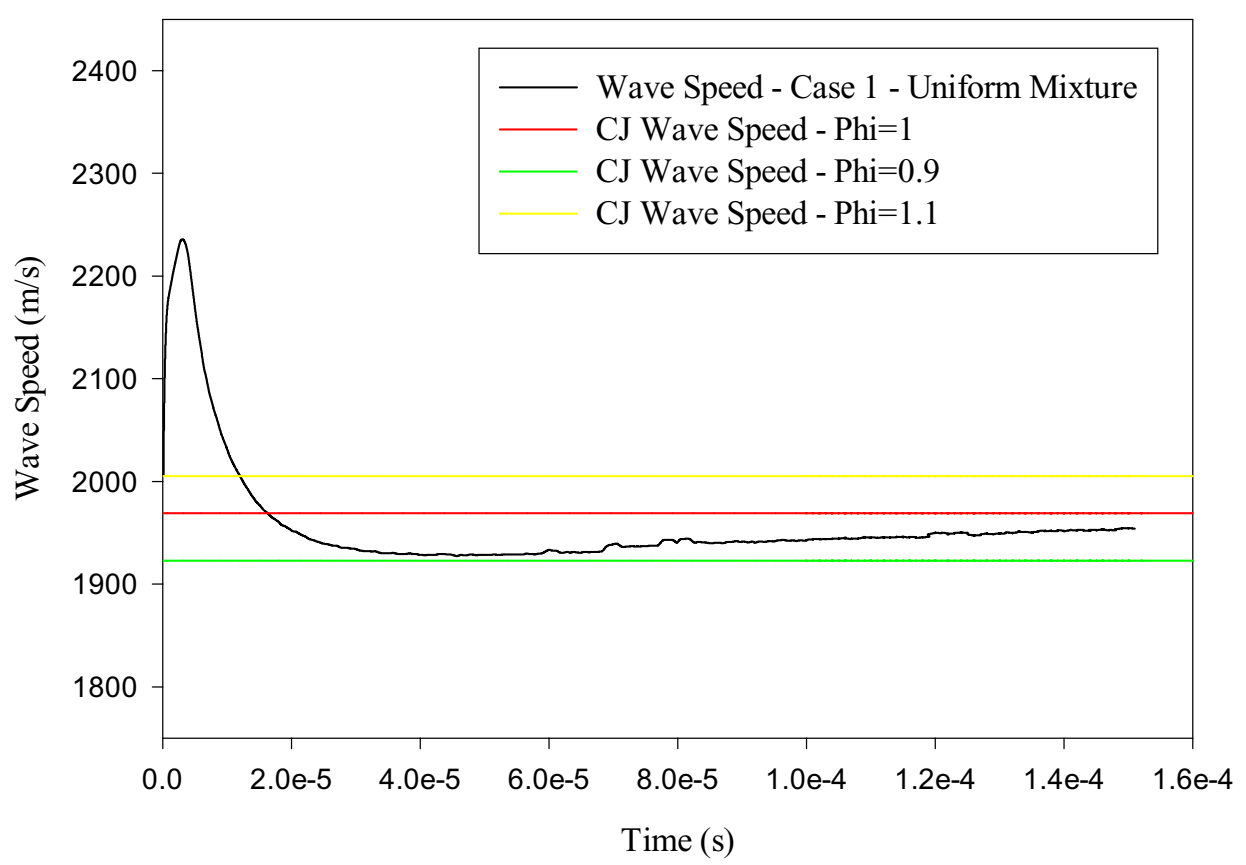

Figure 38 - Case 1 (Uniform Mixture) Wave Speed Compared to Equilibrium CJ Wave Speeds 


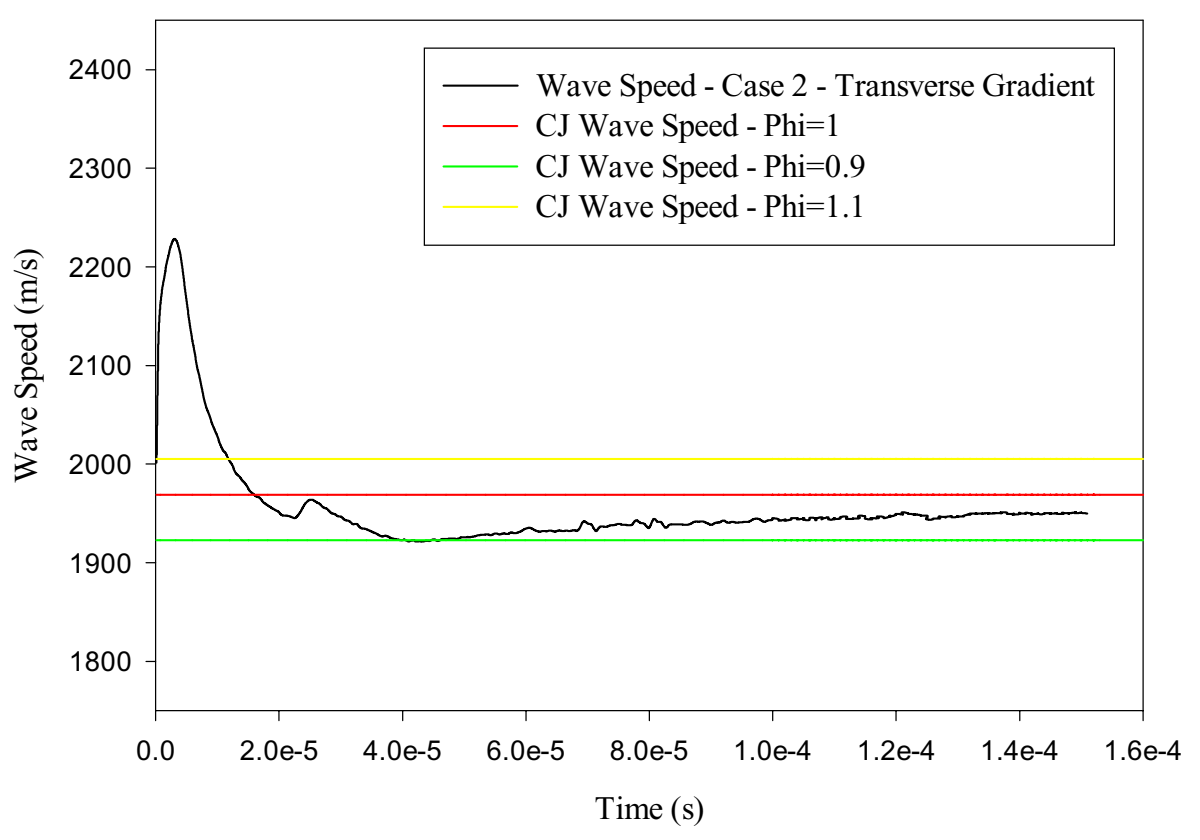

Figure 39 - Case 2 (Transverse Gradient) Wave Speed Compared to Equilibrium CJ Wave Speeds

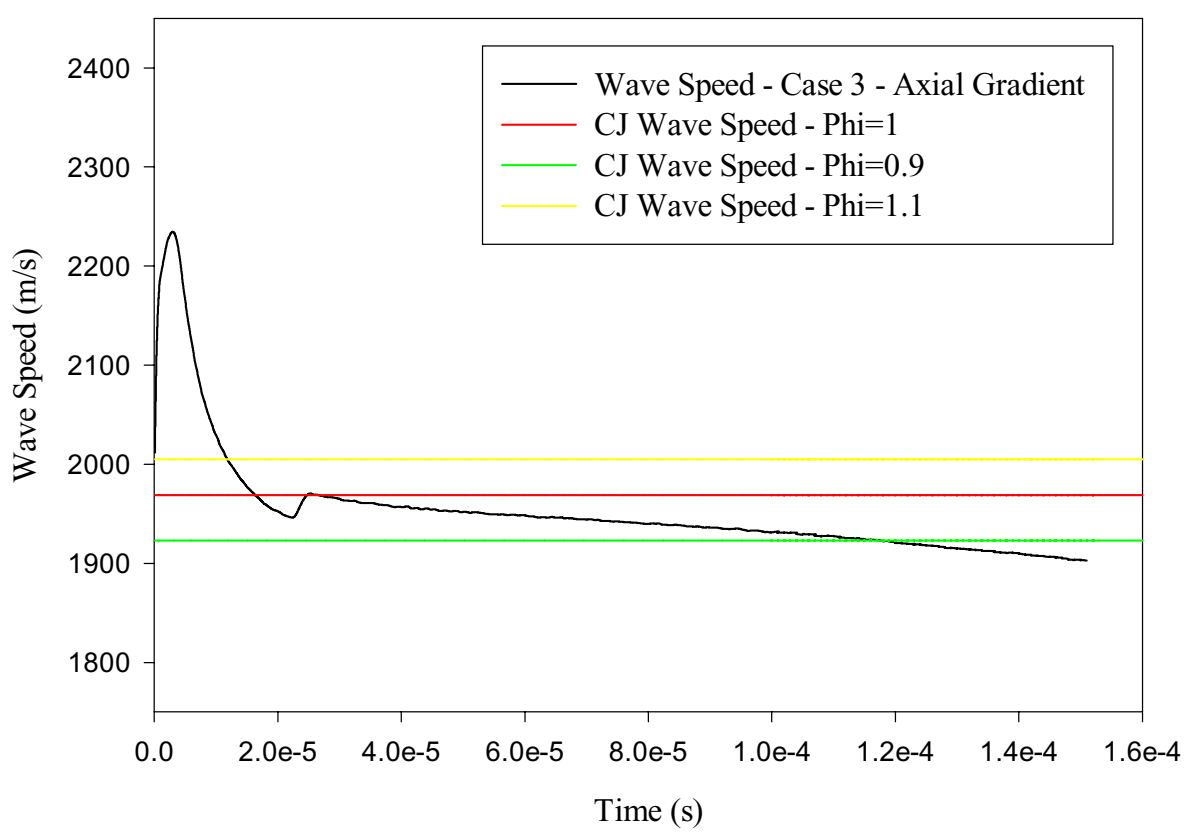

Figure 40 - Case 3 (Axial Gradient) Wave Speed Compared to Equilibrium CJ Wave Speeds 
Figure 41 shows the specific impulse traces versus time for each of the stoichiometrically fueled cases. In the scale of Figure 41, there is essentially no difference in $\mathrm{I}_{\mathrm{sp}}$ across the three simulations. Figure 42 provides a closer view of the final section of the $I_{\text {sp }}$ curves from Figure 41 . We can see that there is some difference in the final values of $\mathrm{I}_{\mathrm{sp}}$, but that the results are all within $1 \%$ of each other, with the axial distribution slightly outperforming the other 2 distributions.

Figure 43 shows that the head-end pressure is essentially unchanged for the three stoichiometric cases. Case 3 shows a slight increase in the head end pressure during the plateau region, consistent with the slightly improved performance shown in Figure 42. The plateau duration and blowdown contour are virtually identical for all three cases.

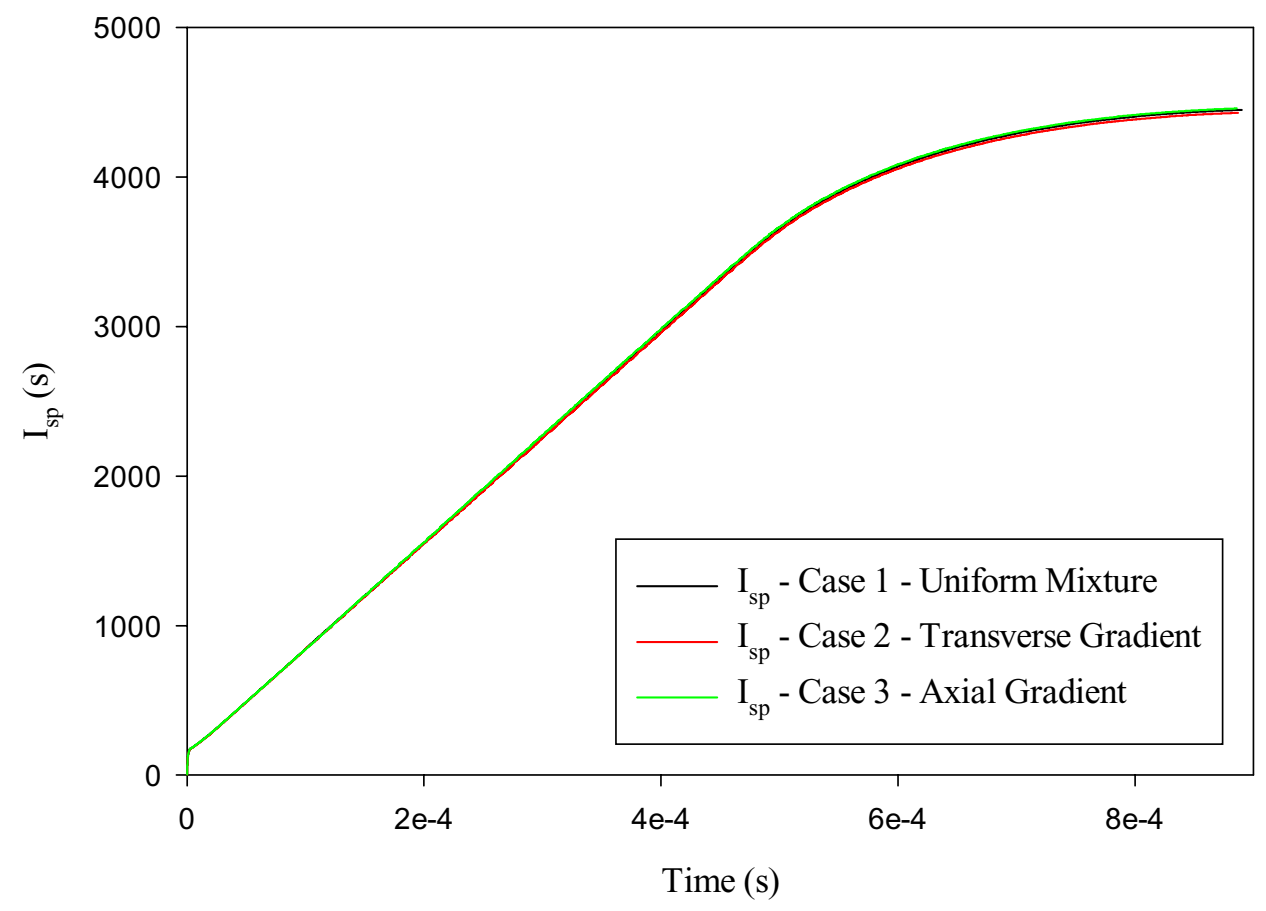

Figure 41 - Specific Impulse of Stoichiometric Cases 1, 2, and 3 


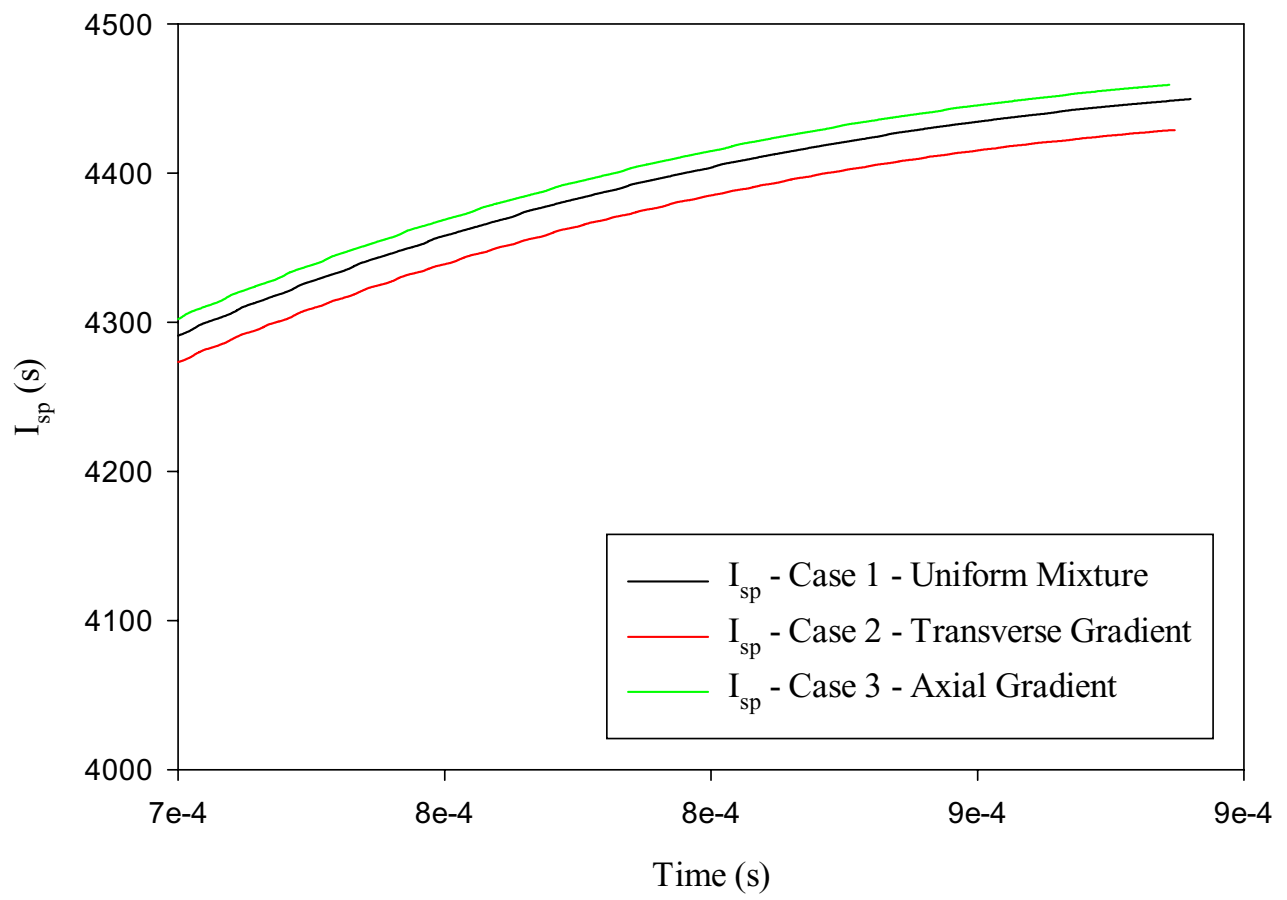

Figure 42 - Enlarged View of Final Specific Impulse of Stoichiometric Cases 1, 2, and 3

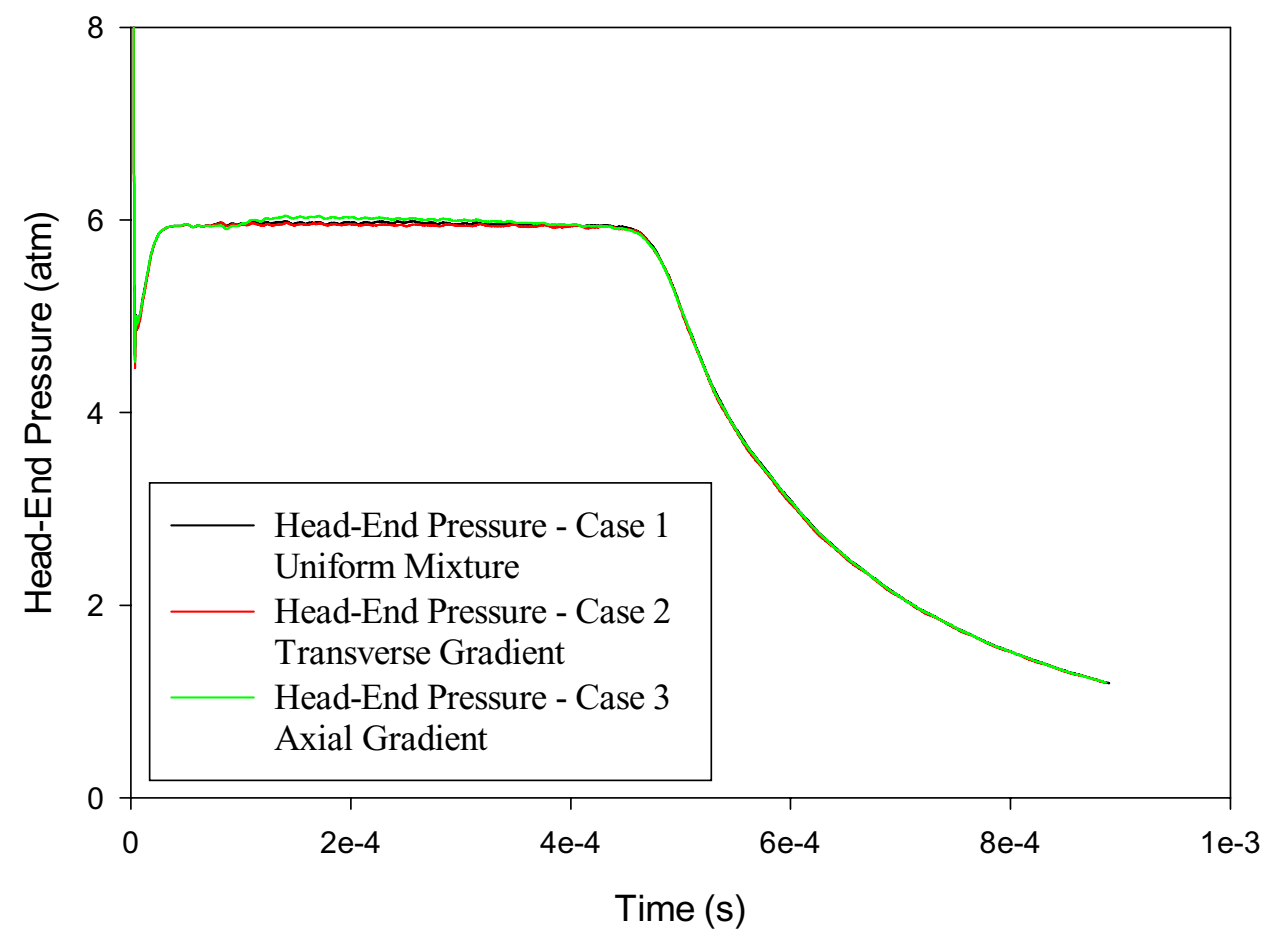

Figure 43 - Head-End Pressure as a Function of Time for Stoichiometric Cases 1, 2, and 3 
Lastly, we can look at plots of $\mathrm{H}_{2}$ or $\mathrm{H}_{2} \mathrm{O}$ mass fraction to determine if any significant mixing or post-detonation combustion is occurring in the detonation tube. Figure 44 shows the hydrogen mass fraction in the tube at five different times during the simulation. The first three plots are during the detonation propagation, and the last two plots are during the blowdown. The post-detonation $\mathrm{H}_{2}$ distribution remains essentially stratified throughout the entire propagation and blowdown, with the composition distribution at the left of the tube stretching to the right to fill the tube as the blowdown progresses. The products of the uniformly fueled stoichiometric stabilization zone eventually push out most of the stratified combustion products by the end of the simulation. The relatively high levels of residual $\mathrm{H}_{2}$ at the left-hand (closed) end of the tube seen in this time sequence are a result of the thermal breakdown of $\mathrm{H}_{2} \mathrm{O}$ from in the high pressure, high temperature region used to initiate the detonation and are not a product of the detonation process itself. It can be assumed that negligible mixing occurs in the axial direction if little mixing is observed in the transverse direction, since the axial composition gradients are approximately $1 / 16^{\text {th }}$ as steep as the transverse composition gradients. This is born out by the one-dimensional time history of $\mathrm{H}_{2} \mathrm{O}$ mass fraction for Case 3 shown in Figure 45.

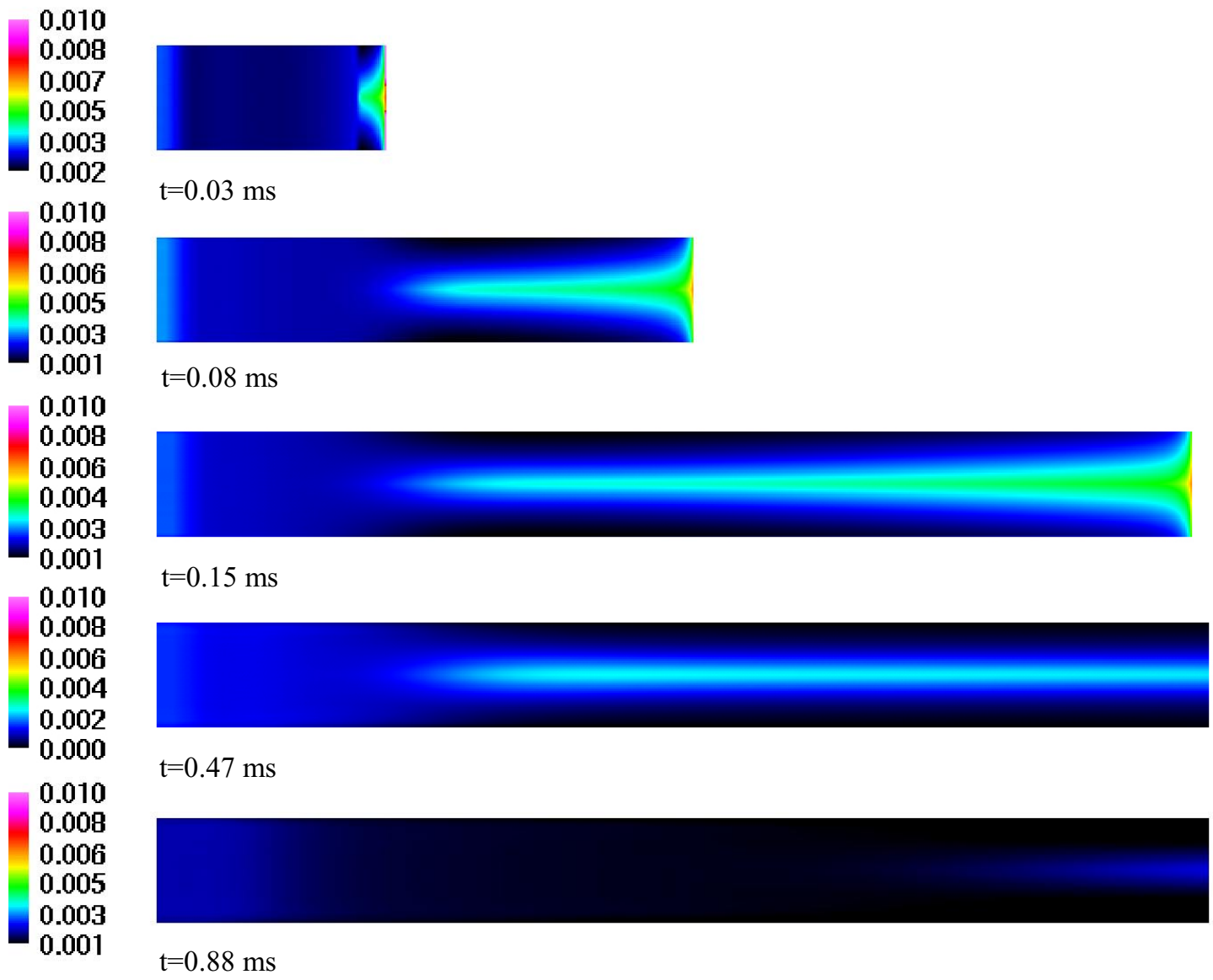

Figure 44 - Two-Dimensional $\mathrm{H}_{2}$ Mass Fraction Distributions for Case 2 Transverse Gradient 


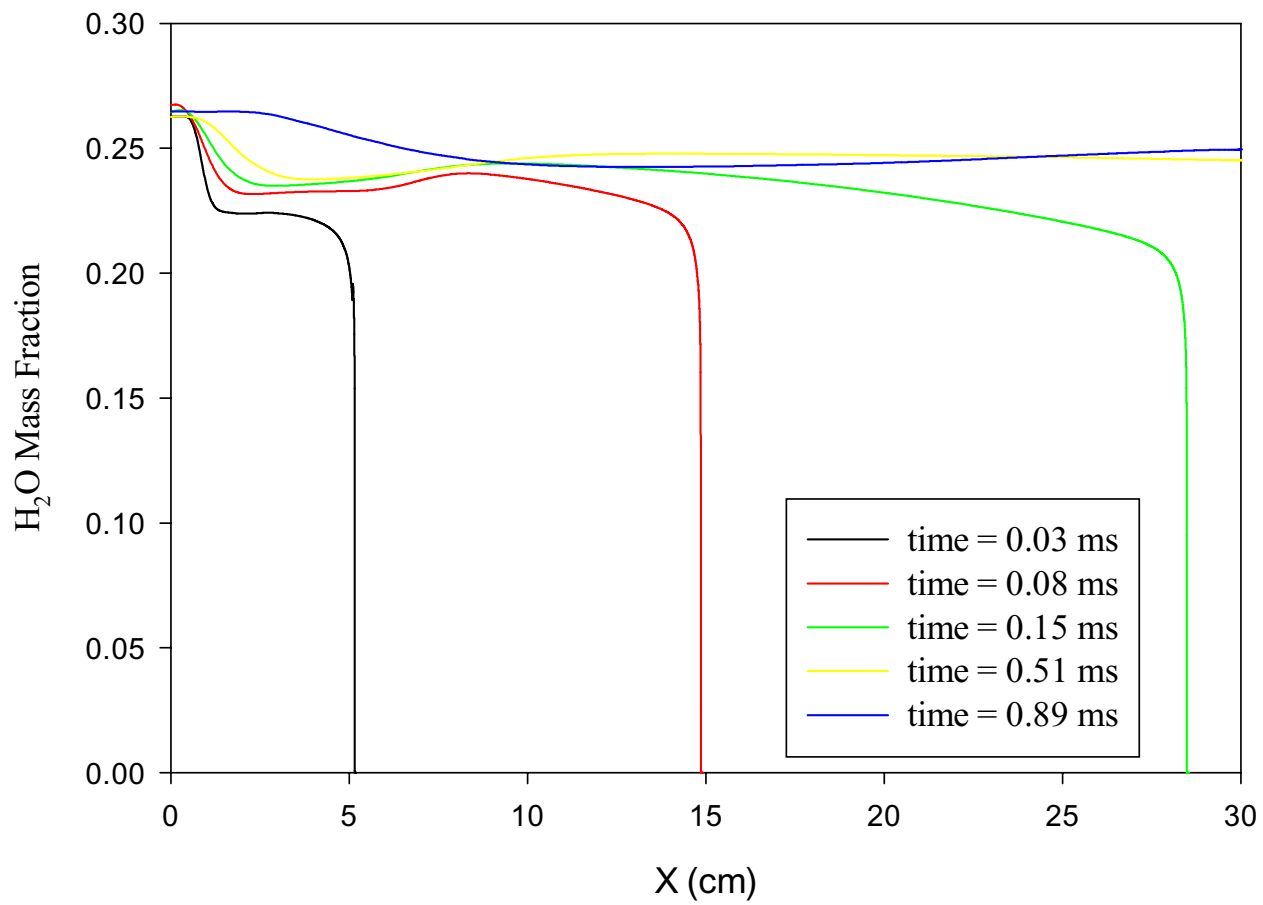

Figure 45 - One-Dimensional $\mathrm{H}_{2} \mathrm{O}$ Mass Fraction Distributions for Case 3 Axial Gradient

\subsection{Fuel Lean Mixtures}

The wave speed results for the fuel lean cases, Figures 46 through 50, are much the same as the stoichiometric cases. The uniformly fueled mixture, Case 4 , and the transversely varying mixture, Case 5, follow a similar profile of wave speed. The axially varying mixture, Case 6 , follows the initial mixture profile at near the equilibrium detonation speed. The added case, a uniform stoichiometric mixture with an air buffer at the end of the tube, shows the expected drop off in wave speed as the detonation fails in the unfueled air. The wave speed falls off precipitously as the detonation wave enters the unfueled region, limiting the transfer of energy to the buffer air region. 


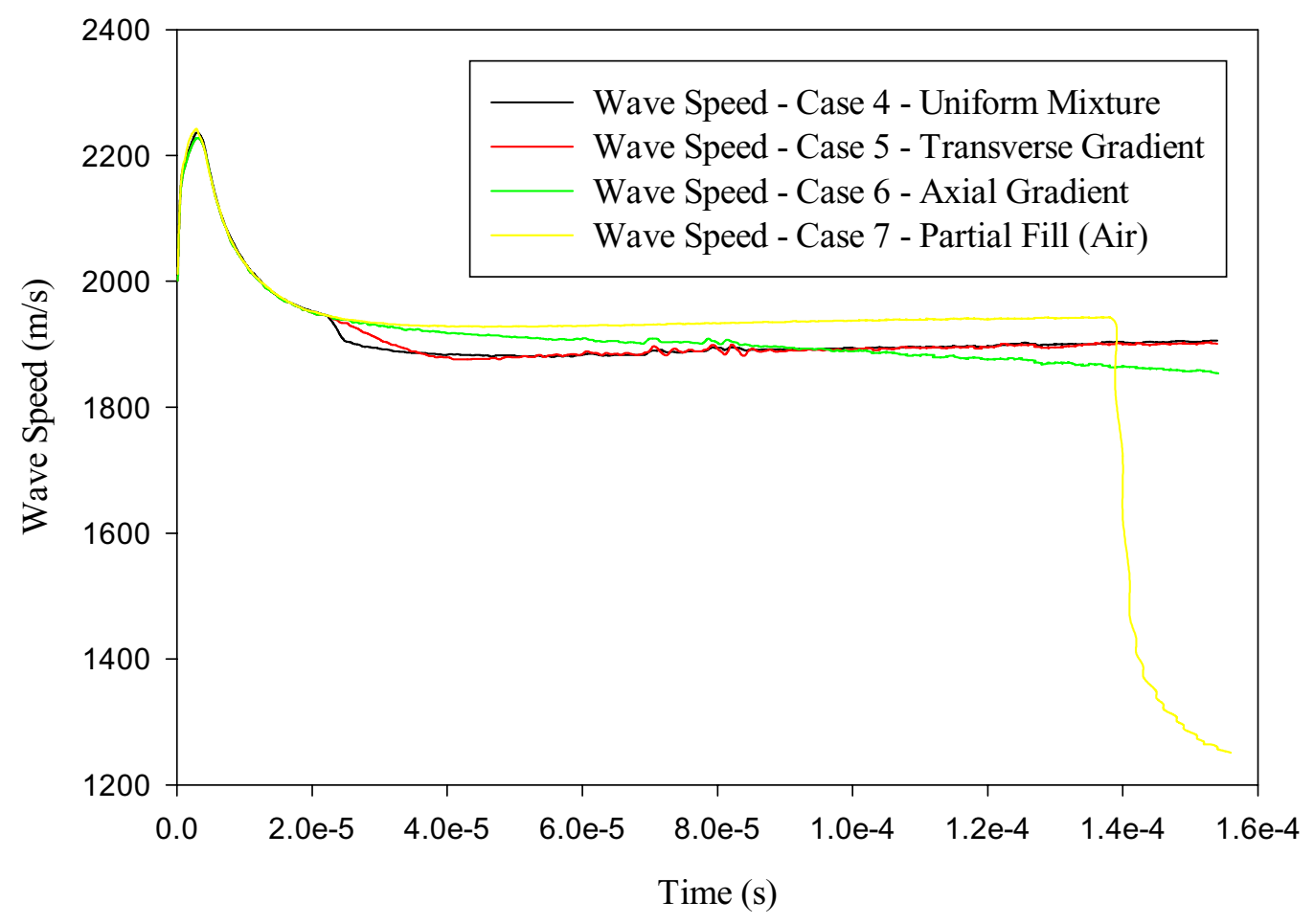

Figure 46 - Detonation Wave Speeds of Fuel Lean Cases 4, 5, 6, and 7

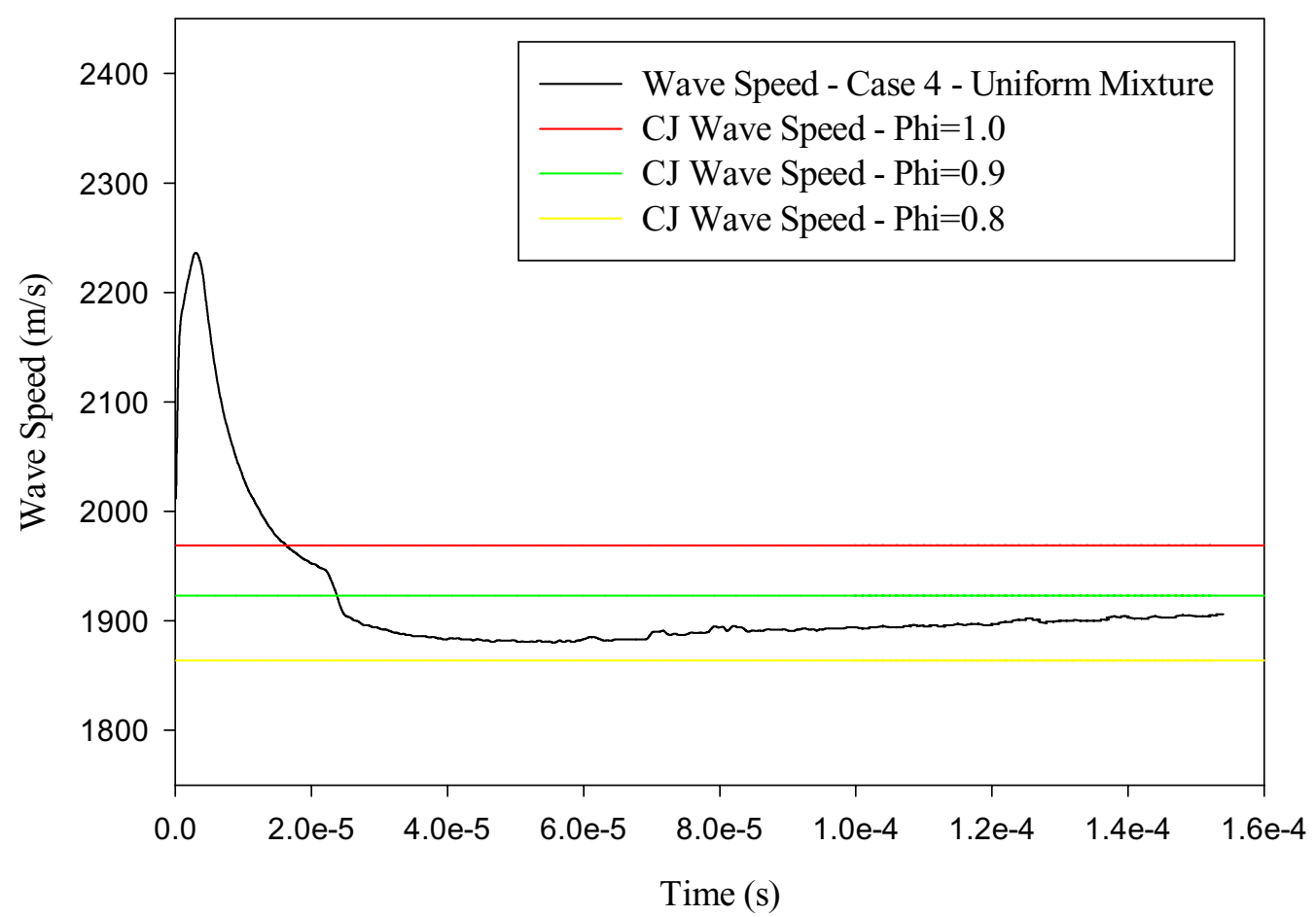

Figure 47 - Case 4 (Uniform Mixture) Wave Speed Compared to Equilibrium CJ Wave Speeds 


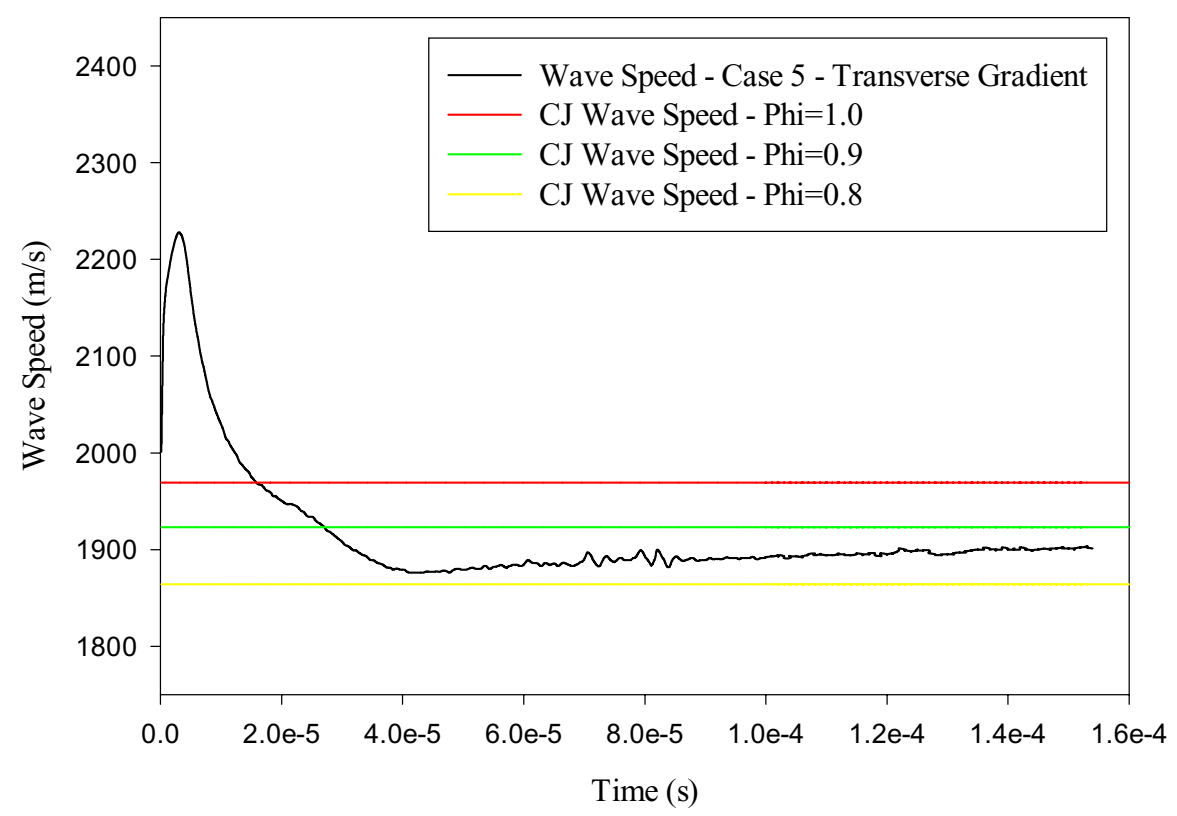

Figure 48 - Case 5 (Transverse Gradient) Wave Speed Compared to Equilibrium CJ Wave Speeds

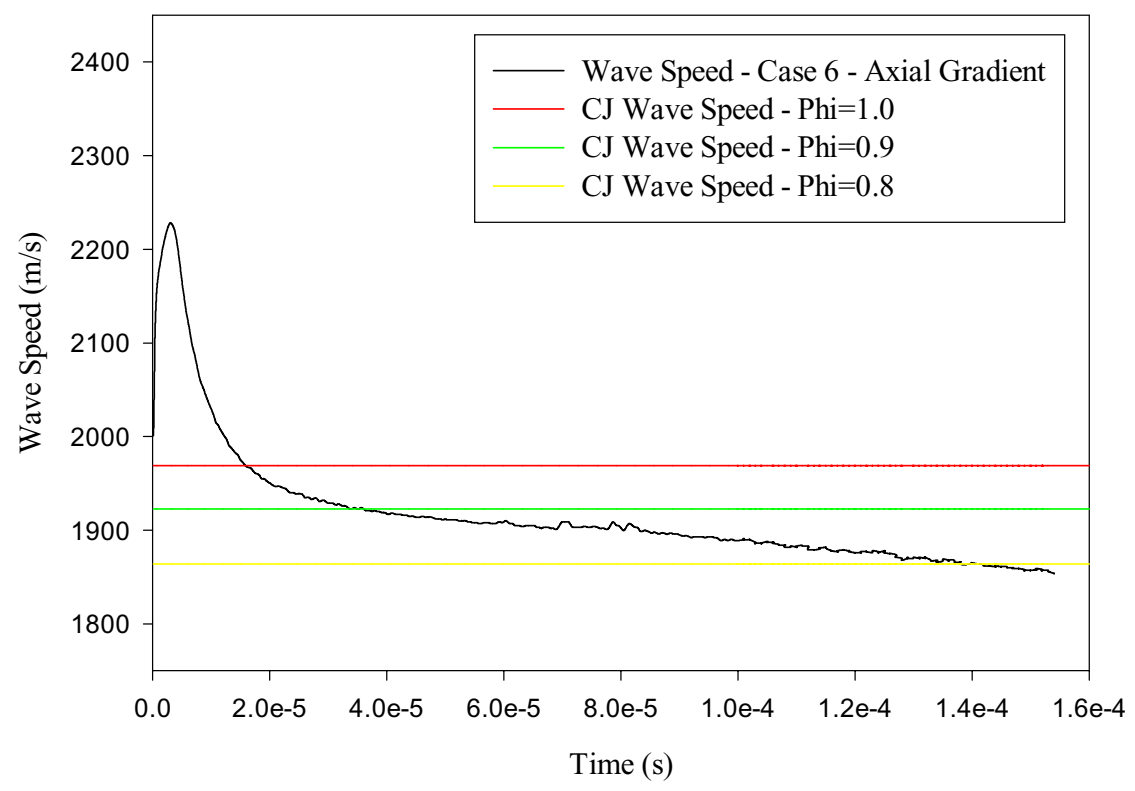

Figure 49 - Case 6 (Axial Gradient) Wave Speed Compared to Equilibrium CJ Wave Speeds 


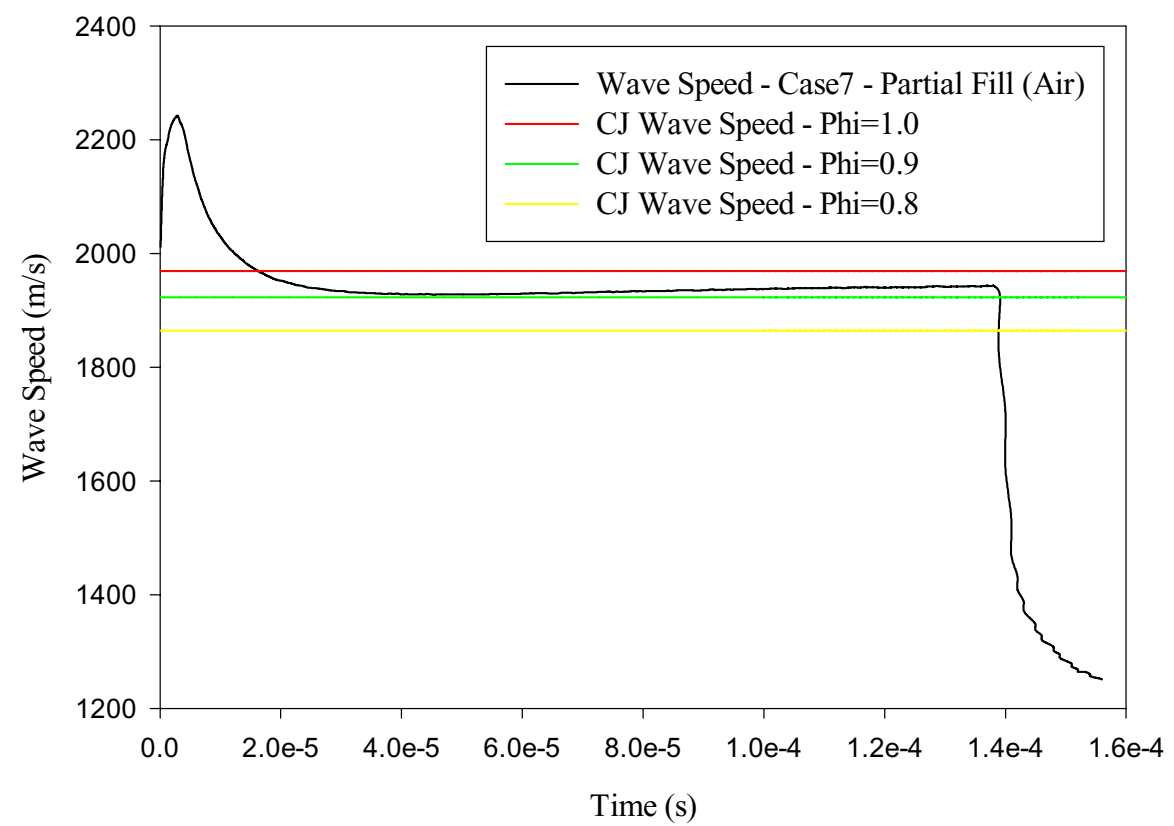

Figure 50 - Case 7 (Partial Fill (Air)) Wave Speed Compared to Equilibrium CJ Wave Speeds

The specific impulse results, Figure 51, are also similar to the stoichiometric results, allowing for the difference in magnitude due to the change in overall equivalence ratio as documented in Section 5.1. The total variation in specific impulse is on the order of $1 \%$. We again see the axial distribution slightly outperforming the uniform and transverse distributions. The new "partial fill" case slightly under-performs the others. This indicates that it would be slightly better to throttle a PDE by decreasing the equivalence ratio through reduced fuel flow rather than by partial filling, if that is a practical approach when other factors are considered.

The head end pressure time histories in Figure 52 show more case-to-case variation than did the stoichiometric mixtures. The axially varying mixture has a continually decreasing plateau pressure, and the partial fill case has a shortened pressure plateau. While there are differences, it is evident from the specific impulse results that the positive and negative aspects of these differences work to cancel each other out. 


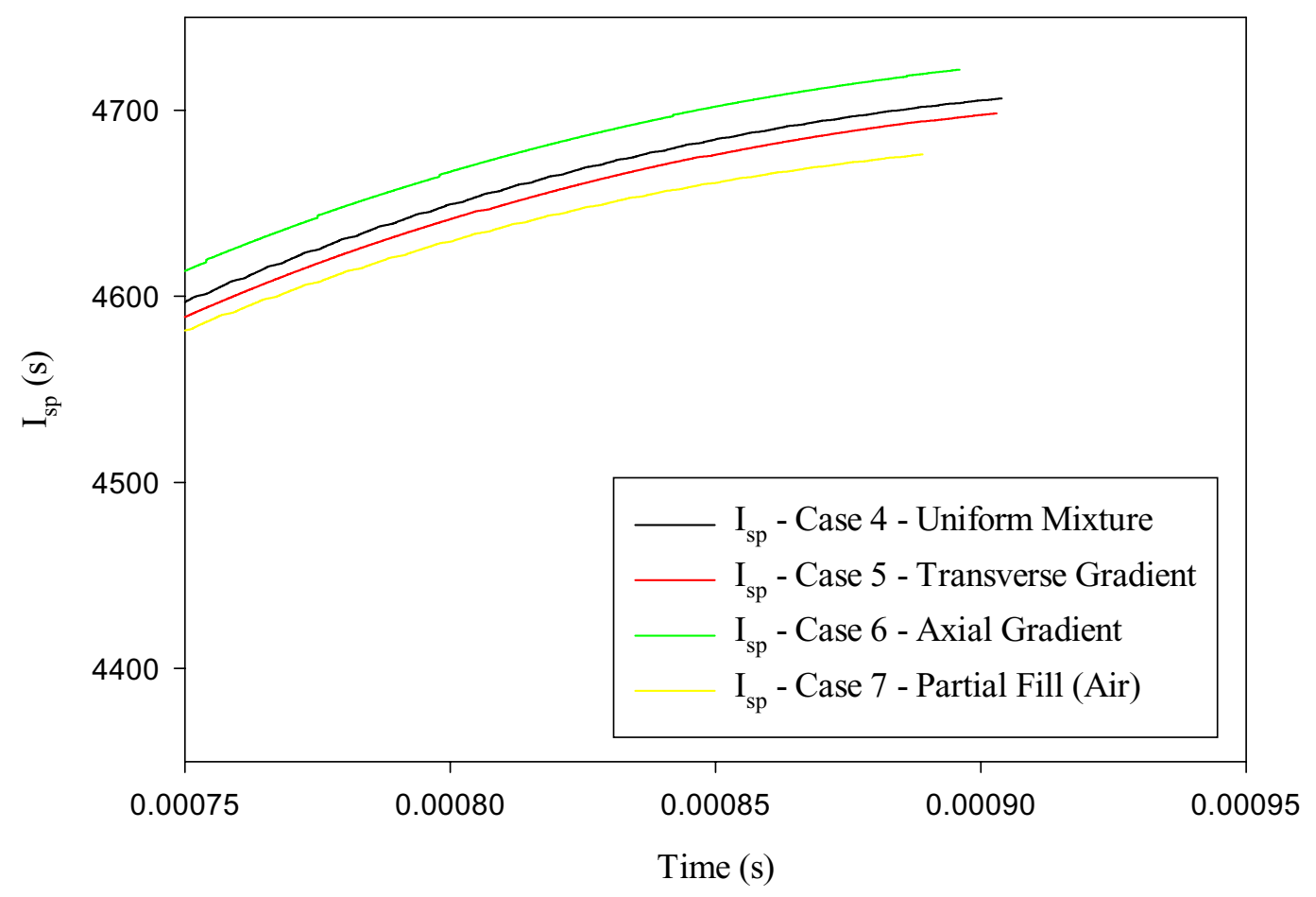

Figure 51 - Enlarged View of Final Specific Impulse of Fuel Lean Cases 4, 5, 6, and 7

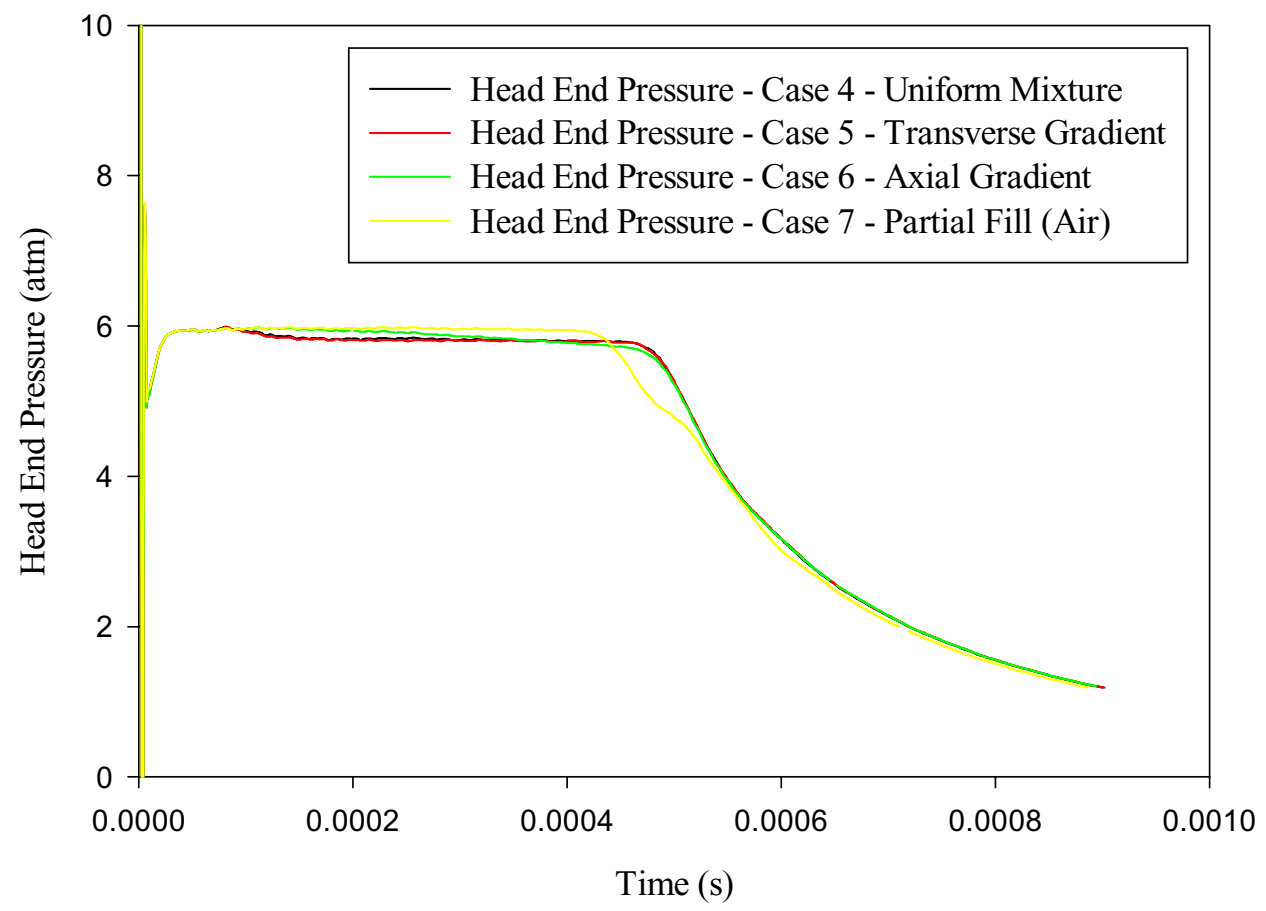

Figure 52 - Head-End Pressure as a Function of Time for Fuel Lean Cases 4, 5, 6, and 7 
Again checking for mixing in the detonation products for the transversely varying mixture, Case 5, Figure 53 shows a time series of two-dimensional plots of $\mathrm{O}_{2}$ mass fraction. Since there are no fuel rich zones in this distribution, it is expected that there will be no more than trace amounts of $\mathrm{H}_{2}$ present in the combustion products, so $\mathrm{O}_{2}$ serves as a better indicator of mixing and/or combustion within the tube. The same characteristic behavior is seen here as was seen for the stoichiometric case. The stratified post-detonation composition distribution remains stratified as it is pushed out of the detonation tube during the blowdown process. No significant mixing or diffusion leading to post-detonation combustion is observed.

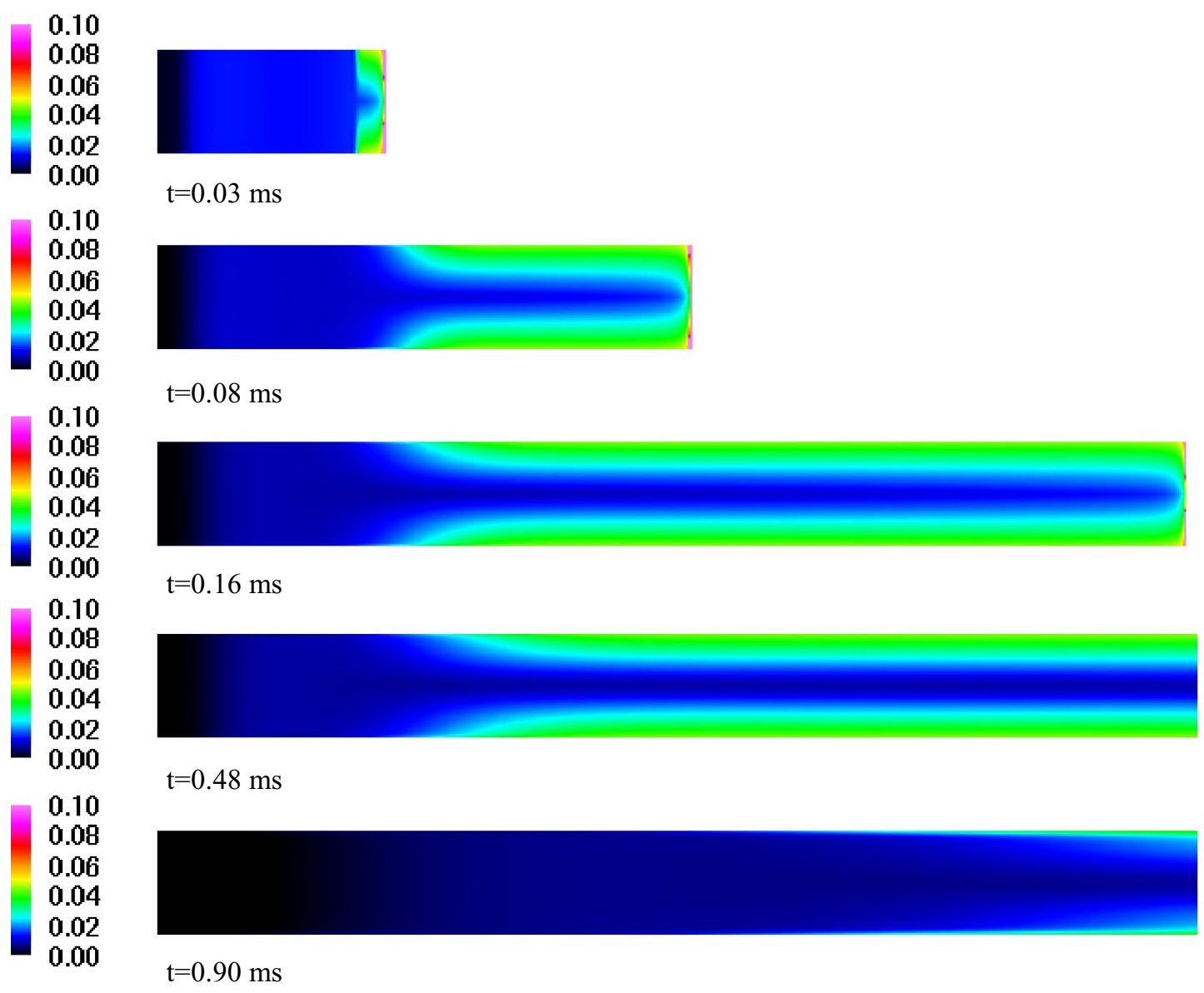

Figure 53 - Two-Dimensional $\mathrm{O}_{2}$ Mass Fraction Distributions for Case 5 Transverse Gradient

\subsection{Fuel Rich Mixtures}

The wave speed results, Figures 54 through 58, for the fuel rich cases also show similar behavior to those for the previous two overall equivalence ratios. The uniformly fueled mixture wave speed, Case 8 , and the transversely varying mixture wave speed, 


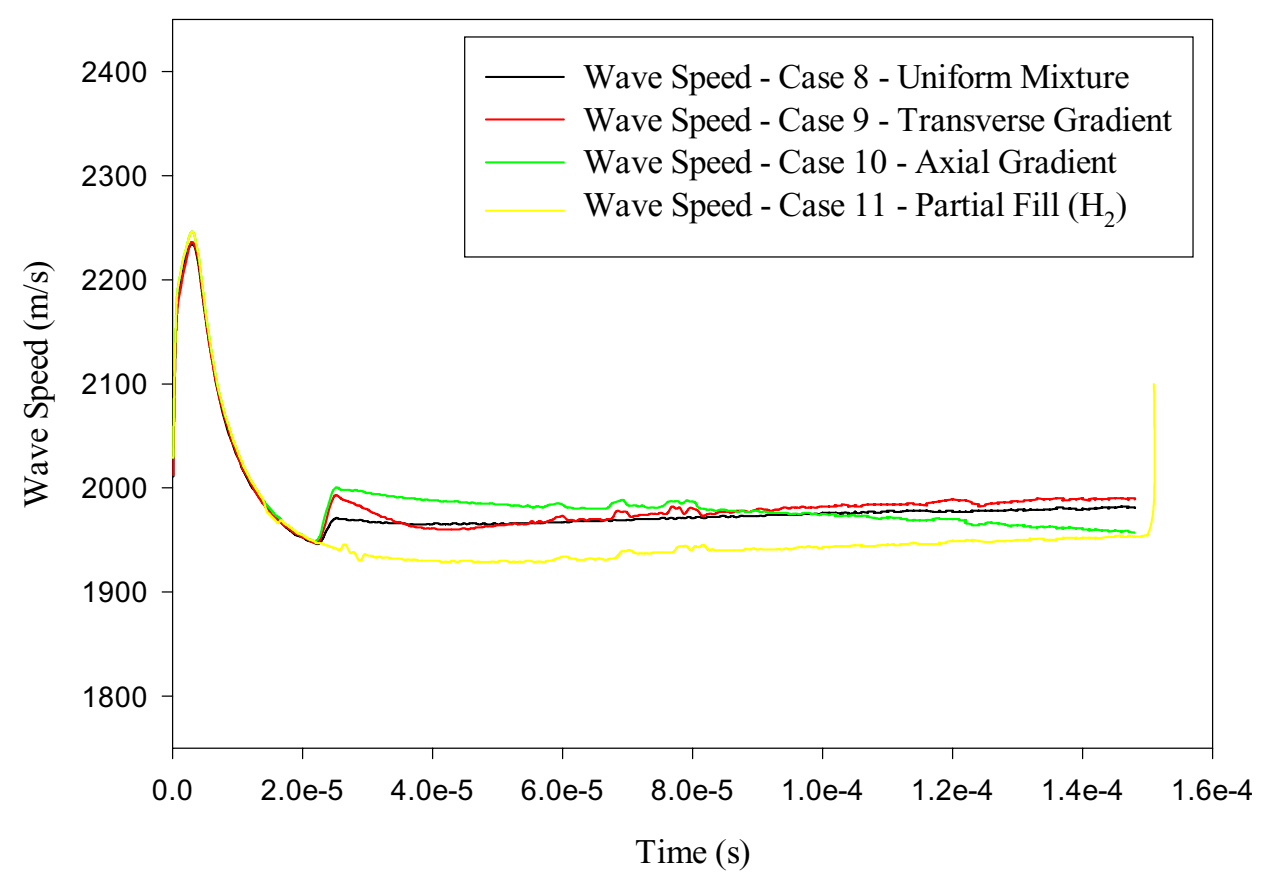

Figure 54 - Detonation Wave Speeds of Fuel Rich Cases 8, 9, 10, and 11

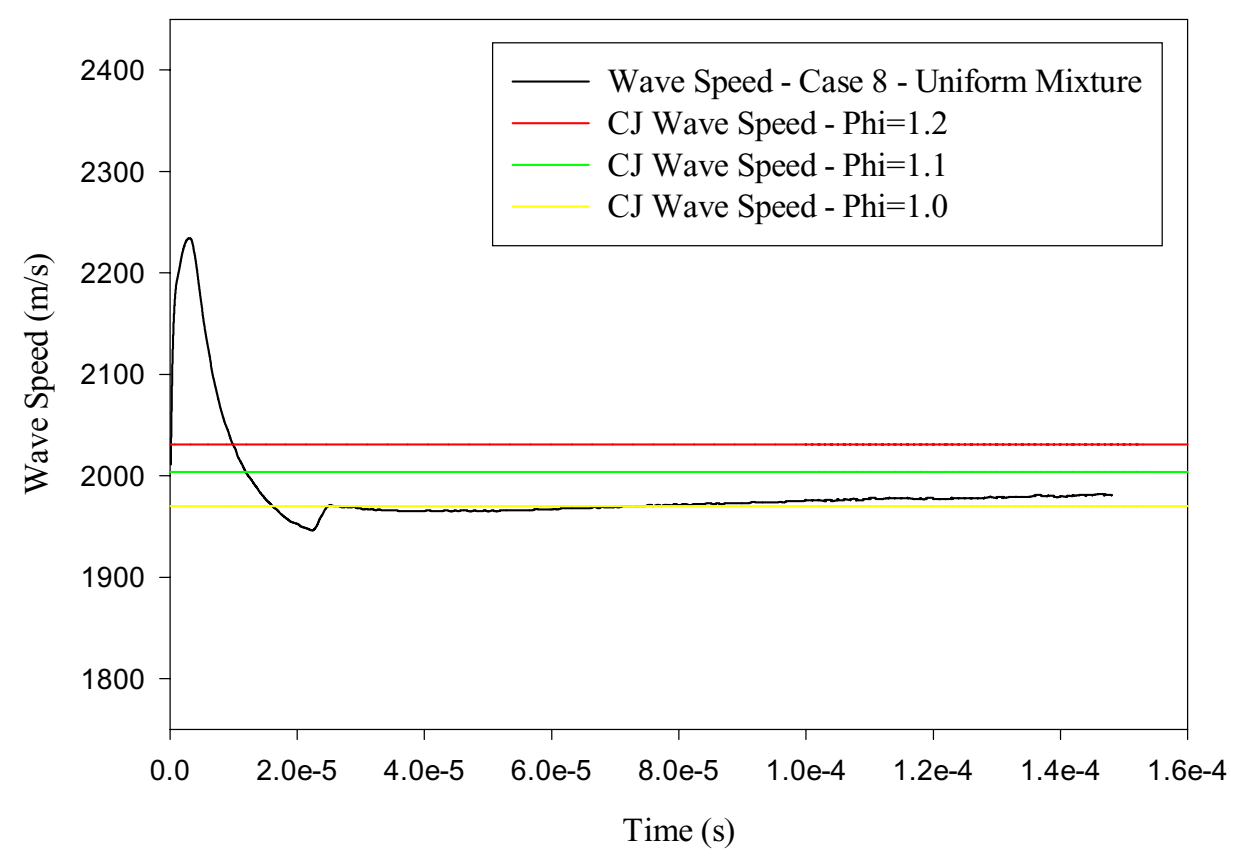

Figure 55 - Case 8 (Uniform Mixture) Wave Speed Compared to Equilibrium CJ Wave Speeds 


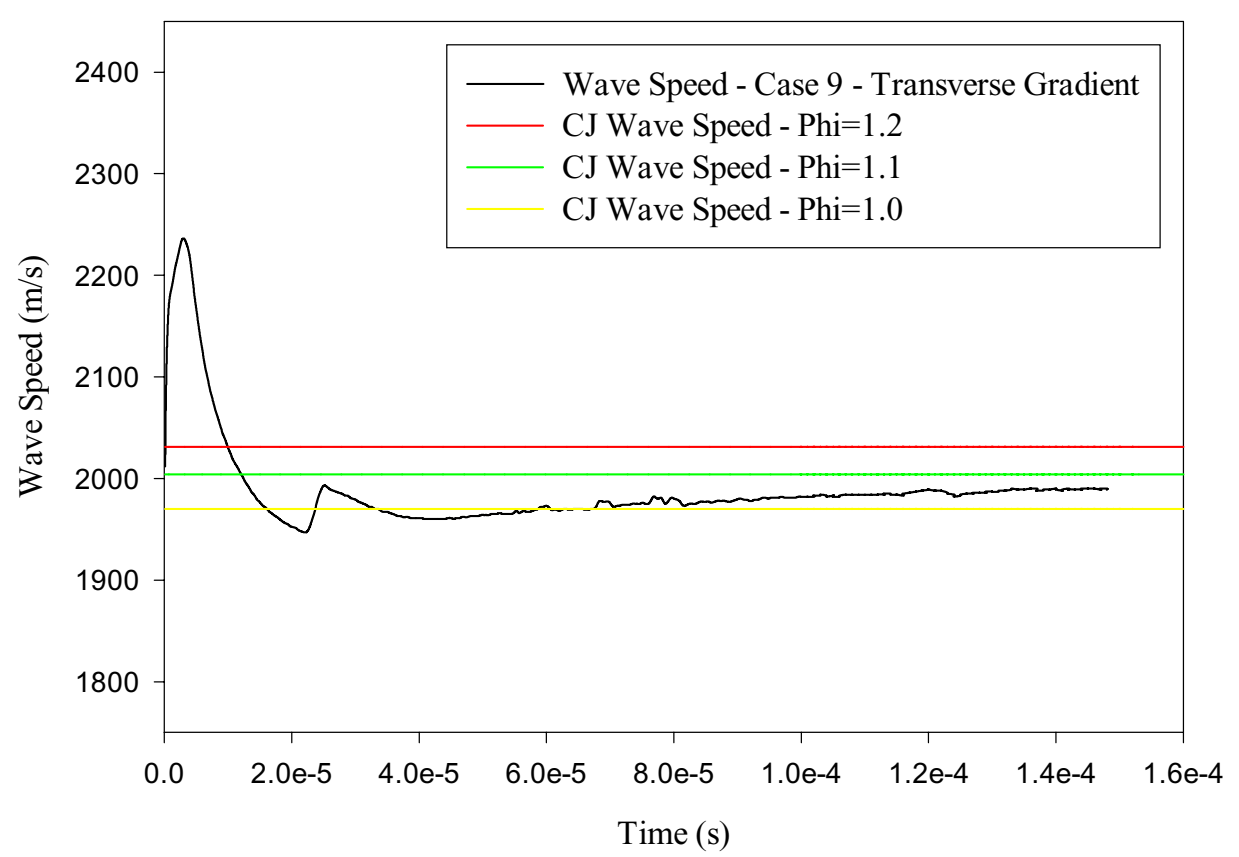

Figure 56 - Case 9 (Transverse Gradient) Wave Speed Compared to Equilibrium CJ Wave Speeds

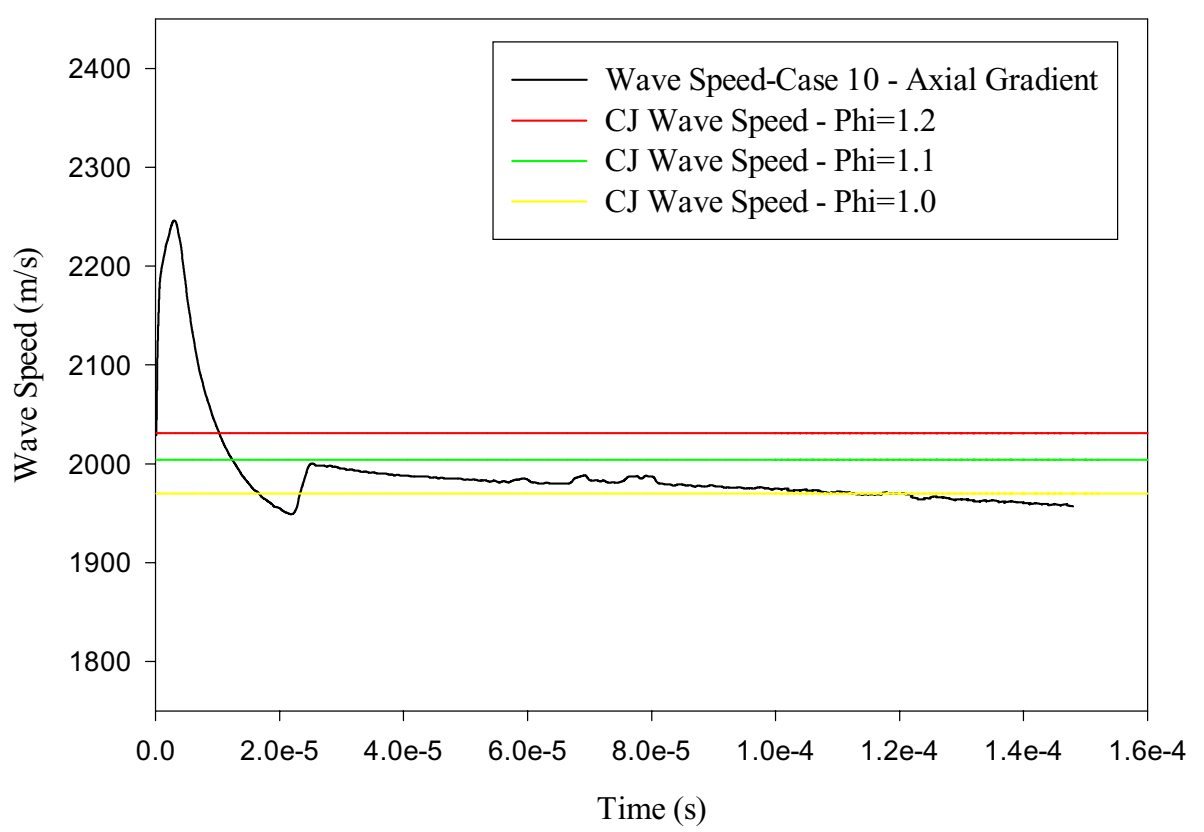

Figure 57 - Case 10 (Axial Gradient) Wave Speed Compared to Equilibrium CJ Wave Speeds 


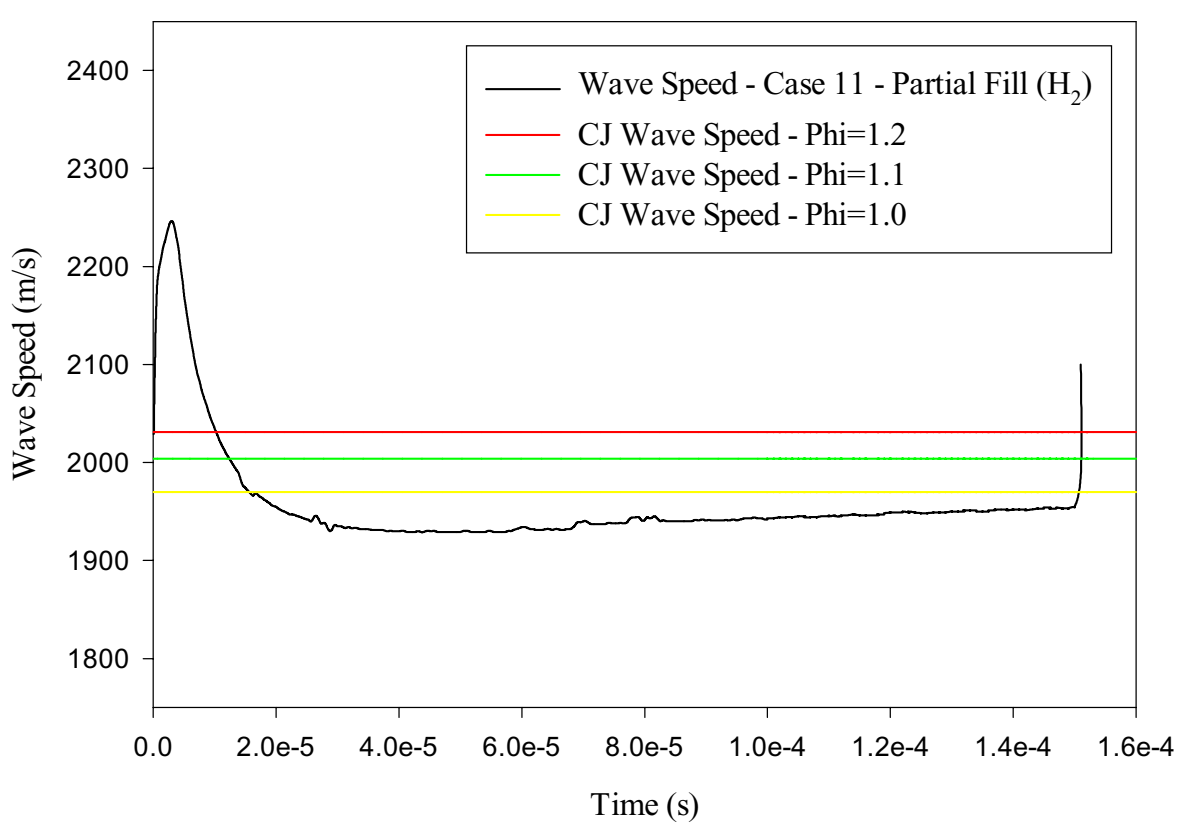

Figure 58 - Case 11 (Partial Fill $\left(\mathrm{H}_{2}\right)$ ) Wave Speed Compared to Equilibrium CJ Wave Speeds

Case 9, do not agree quite as well as before, but are still relatively close. The axially varying mixture wave speed, Case 10, still follows the initial mixture distribution. The partial fill distribution, Case 11, this time with $\mathrm{H}_{2}$ at the end of the tube instead of air, shows a marked increase in wave speed as the detonation wave enters the end buffer region. This is due to the change in the local speed of sound that is significantly higher in hydrogen than in air.

The specific impulse results (Figure 59) are, if anything, more tightly clustered than in either of the previous groups of mixtures. The uniformly fueled case and the axially varying case are almost identical, with the transversely varying case slightly lower. The partial fill case has gone from being the worst performer with an air buffer for the fuel lean cases, to being the best performer for the fuel rich cases. However, again the total variation in results is on the order of only $1 \%$.

Looking at the head end pressure time histories in Figure 60, we see almost no variation at all, despite the slightly increased variability in wave speed observed in Figure 54.

Lastly, we see in Figure 61 the same lack of mixing in the post-detonation composition distribution of the 2-dimensional plots for the transverse mixture in Case 9. $\mathrm{H}_{2}$ mass fraction has again been used for these plots since there are fuel rich zones in the pre-combustion mixture. 


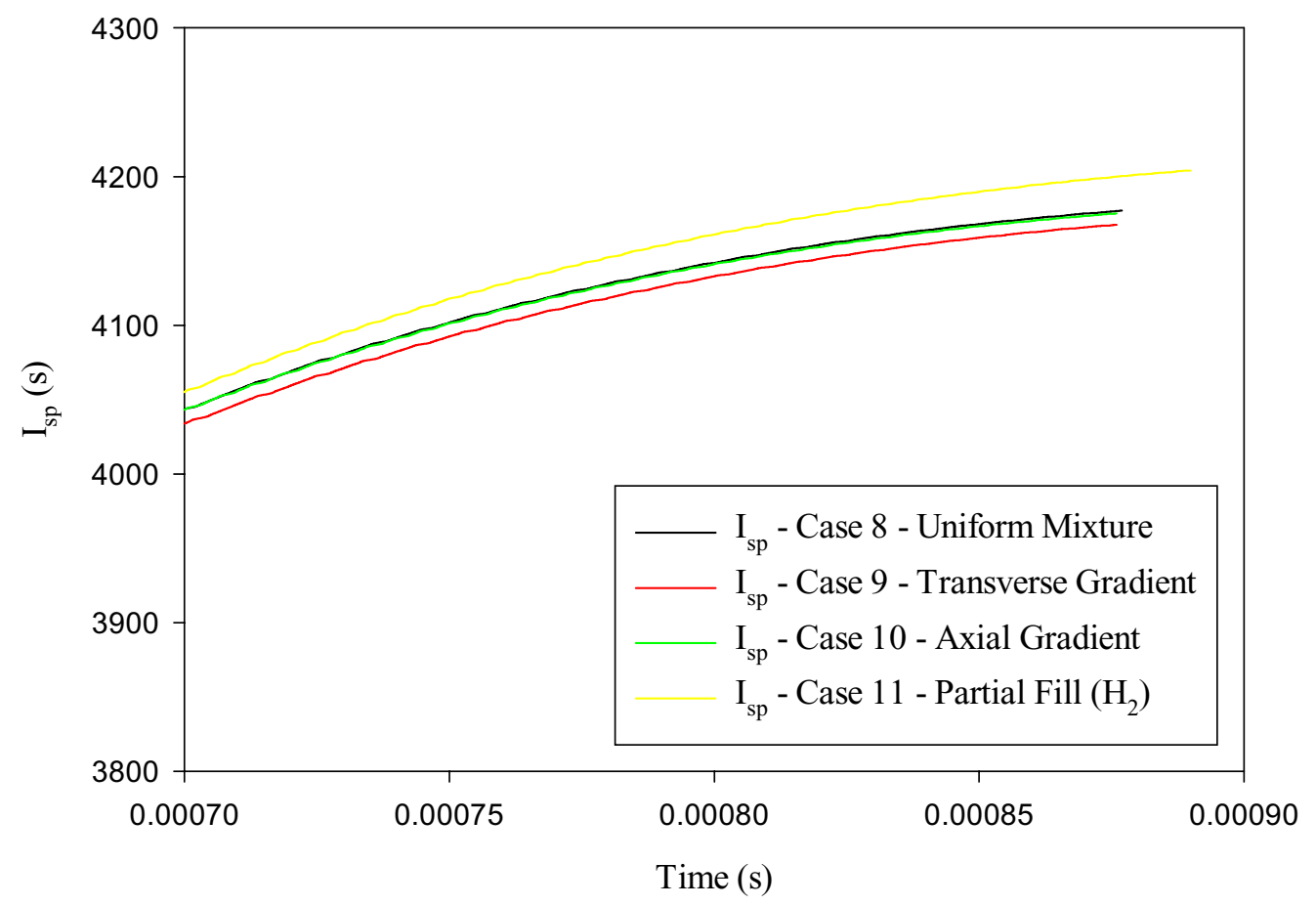

Figure 59 - Enlarged View of Final Specific Impulse of Fuel Rich Cases 8, 9, 10, and 11

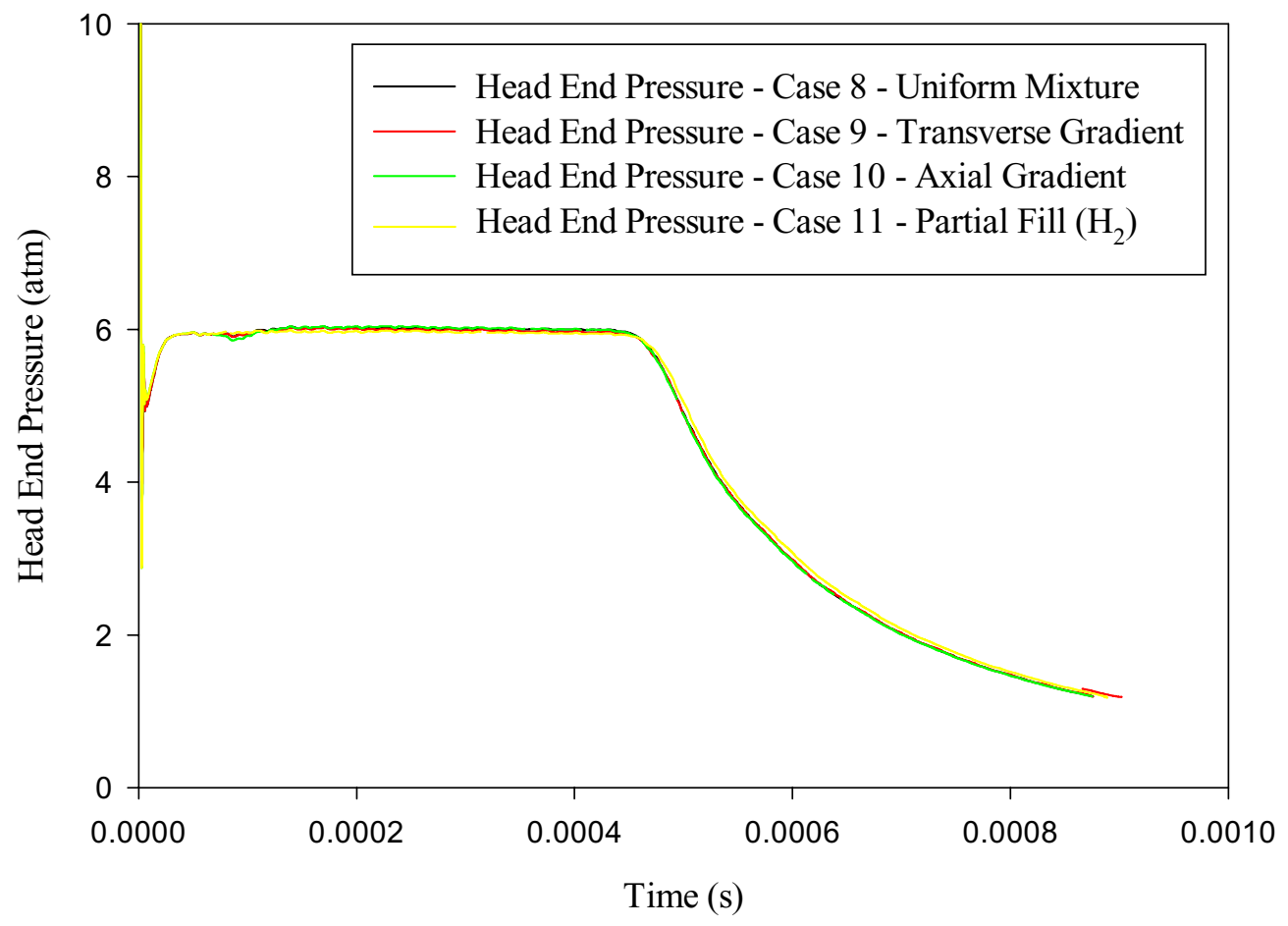

Figure 60 - Head-End Pressure as a Function of Time for Fuel Rich Cases 8, 9, 10, and 11 


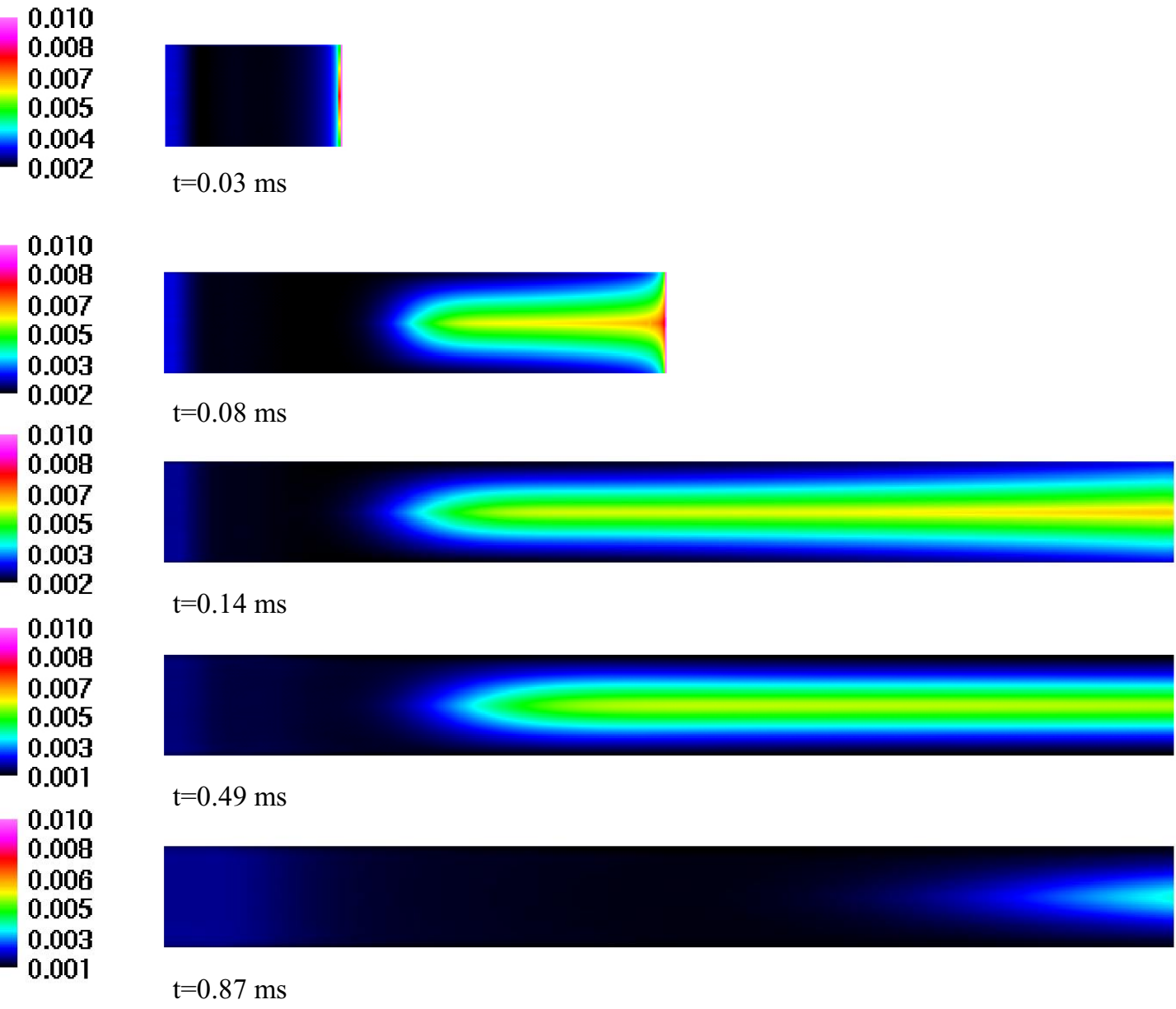

Figure $61-\mathrm{H}_{2}$ Mass Fraction Distributions for Case 9 - Transverse Gradient

\section{Summary and Conclusions}

\subsection{Summary of Results}

A total of eleven 2-dimensional detonation tube simulations were conducted. Of these, three were run with stoichiometric $\mathrm{H}_{2} /$ air mixtures, four were run with fuel lean $\mathrm{H}_{2}$ /air mixtures, and four were run with fuel rich $\mathrm{H}_{2}$ /air mixtures. An approximately linear relationship between $\mathrm{I}_{\mathrm{sp}}$ and equivalence ratio was observed between the uniformly fueled cases $(1,4$ and 8$)$. Very little variation $(<1 \%)$ in $\mathrm{I}_{\mathrm{sp}}$ was observed between cases of the same overall stoichiometry. These results are summarized in Table 2. 
Table 2 - Summary of Test Case Performance Results

\begin{tabular}{|c|c|c|c|}
\hline $\begin{array}{c}\text { Case } \\
\text { Number }\end{array}$ & $\begin{array}{c}\text { Overall } \\
\text { Stoichiometry }\end{array}$ & $\begin{array}{c}\text { Type of } \\
\text { Distribution }\end{array}$ & $\begin{array}{c}\mathrm{I}_{\mathrm{sp}} \\
(\mathrm{s})\end{array}$ \\
\hline 1 & Stoichiometric & Uniform & 4450 \\
\hline 2 & Stoichiometric & Transverse & 4429 \\
\hline 3 & Stoichiometric & Axial & 4459 \\
\hline 4 & Fuel Lean & Uniform & 4706 \\
\hline 5 & Fuel Lean & Transverse & 4699 \\
\hline 6 & Fuel Lean & Axial & 4722 \\
\hline 7 & Fuel Lean & Partial Fill (Air) & 4676 \\
\hline 8 & Fuel Rich & Uniform & 4177 \\
\hline 9 & Fuel Rich & Transverse & 4168 \\
\hline 10 & Fuel Rich & Axial & 4175 \\
\hline 11 & Fuel Rich & Partial Fill ( $\left.\mathrm{H}_{2}\right)$ & 4204 \\
\hline
\end{tabular}

Transversely and axially varying fuel/air mixtures remained basically stratified throughout the detonation propagation and blowdown process.

\subsection{Conclusions}

The primary conclusion to be drawn from this study is that a lack of fuel-air mixing up to a moderate level in a hydrogen fueled airbreathing detonation tube has almost no effect on the thrust performance of the system. Put another way, thrust performance is almost completely independent of combustion efficiency in the vicinity of an overall equivalence ratio of one. This is an encouraging result for the design of such systems, as it appears to be unnecessary to go to extremes to achieve good mixing in the fuel injection process. These results also imply that it is probably unnecessary to match air and fuel valve opening and closing profiles to maintain constant equivalence ratio. These results are limited to those cases where the entire fuel/air charge, while not fully mixed, is nonetheless fully detonable. 
There are a number of secondary conclusions that may also be drawn for $\mathrm{H}_{2} /$ air systems.

1. Decreased equivalence ratio and partial-filling of the detonation tube are essentially equivalent. If anything, it appears slightly more advantageous to lower the equivalence ratio as long as the mixture remains detonable. Obviously, a point is reached where it is impossible to lower the equivalence ratio and maintain a detonable mixture. At this point, further throttling can be achieved by partially filling the detonation tube with a lean mixture.

2. Non-transversely varying mixtures may be treated as one-dimensional, ignoring wall shear losses. This means that future studies with such mixtures may be safely conducted using one-dimensional codes without fear of significant loss of accuracy. This will save considerable time in computational analysis.

3. Transversely and axially varying mixtures remain essentially stratified throughout the detonation propagation and blowdown process. One benefit of this observation is that it will be possible to qualitatively evaluate mixing within a detonation tube by looking at the distribution of products coming out of the tube, thus reducing the need for costly optical test sections in detonation tubes.

4. $\mathrm{H}_{2} /$ air detonation tube systems can be adequately computed using simplified chemistry, thus reducing required computational time. Early test runs using the SPARK code with the full chemical mechanism found in Table A1 showed a greater than one order of magnitude increase in computational time required for a detonation tube calculation. This was due not only to the increased number of species, but also the finer axial grid spacing required to capture with precision the trace concentrations of $\mathrm{HO}_{2}$ and $\mathrm{H}_{2} \mathrm{O}_{2}$ in the reactions subsequently eliminated.

\subsection{Recommendations for Future Work}

There are a number of areas worthy of further inquiry that remain at the conclusion of this study. These include,

Increasing the range of equivalence ratios studied. It would be of great value to study levels of fuel/air mixture variation that included marginally-detonable and non-detonable mixtures. Such mixtures would result from detonation tube film cooling schemes or poor mixing configurations.

1. Performing calculations using a representative hydrocarbon fuel. Hydrocarbon fuels are likely candidates for most non-access-to-space applications due to their high density and ease of use. It is likely that such systems will behave significantly differently. Hydrogen is an excellent monopropellant because of its low molecular weight. Therefore, unburned hydrogen raised to a high temperature by the leading shock wave of the detonation front still provides significant impulse. A hydrocarbon fuel will not behave in the same manner and will likely penalize the system to a greater extent. 
2. Further development of PDEs is likely to bring forward a number of injection patterns not addressed by this study. As realistic patterns become available, further analysis should be performed.

3. Simulations including turbulence should be performed to assess the impact of turbulence, both before and after the passage of the detonation wave, on the overall system performance. 
Appendix

Justification of Truncated Kinetic Mechanism

The standard $\mathrm{H}_{2}$-air kinetic mechanism of Jachimowski (1988) is given in Table A1 below. For this study, reactions 8 through 18 have been eliminated. These reactions are those that contain $\mathrm{HO}_{2}$ and $\mathrm{H}_{2} \mathrm{O}_{2}$, species that generally govern the low temperature ignition process. However, ignition in a detonation wave occurs immediately after the moving shock wave, so the combustible mixture at ignition is not at a low temperature. For an equilibrium stoichiometric $\mathrm{H}_{2}$-air detonation wave with initial conditions of 1 atmosphere and $300 \mathrm{~K}$, the Gordon and McBride (1994) CEA code predicts a detonation wave Mach number of 4.8. This corresponds to a temperature and pressure behind the shock of approximately $1650 \mathrm{~K}$ and 27 atmospheres, respectively, as calculated using the CEA code shock option. It is therefore in the neighborhood of this temperature and pressure that the necessity of incorporating the $\mathrm{HO}_{2}$ and $\mathrm{H}_{2} \mathrm{O}_{2}$ reactions should be considered.

Table A1 - 18-Step $\mathrm{H}_{2}$-Air Mechanism

\begin{tabular}{|c|c|c|c|c|}
\hline $\begin{array}{l}\text { Reaction } \\
\text { Ref.\# }\end{array}$ & Reaction & $\mathrm{A}$ & $\mathrm{n}$ & $\begin{array}{l}\mathrm{Ea} \\
\text { (cal/g-mole) }\end{array}$ \\
\hline 1 & $\mathrm{H}_{2}+\mathrm{O}_{2} \Leftrightarrow 2 \mathrm{OH}$ & $0.1700 \mathrm{e} 14$ & 0.00 & 48150 \\
\hline 2 & $H+O_{2} \Leftrightarrow O H+O$ & $0.1420 \mathrm{e} 15$ & 0.00 & 16400 \\
\hline 3 & $\mathrm{H}_{2}+\mathrm{OH} \Leftrightarrow \mathrm{H}_{2} \mathrm{O}+\mathrm{H}$ & $0.3160 \mathrm{e} 08$ & 1.80 & 3030 \\
\hline 4 & $\mathrm{O}+\mathrm{H}_{2} \Leftrightarrow \mathrm{OH}+\mathrm{H}$ & $0.2070 \mathrm{e} 15$ & 0.00 & 13750 \\
\hline 5 & $\mathrm{OH}+\mathrm{OH} \Leftrightarrow \mathrm{H}_{2} \mathrm{O}+\mathrm{O}$ & $0.5500 \mathrm{e} 14$ & 0.00 & 7000 \\
\hline 6 & $\mathrm{OH}+\mathrm{H}+\mathrm{M} \Leftrightarrow \mathrm{H}_{2} \mathrm{O}+\mathrm{M}$ & $0.2210 \mathrm{e} 23$ & -2.00 & 0 \\
\hline 7 & $H+H+M \Leftrightarrow H_{2}+M$ & $0.6530 \mathrm{e} 18$ & -1.00 & 0 \\
\hline 8 & $H+\mathrm{O}_{2}+M \Leftrightarrow H O_{2}+M$ & $0.3200 \mathrm{e} 19$ & -1.00 & 0 \\
\hline 9 & $\mathrm{OH}+\mathrm{HO}_{2} \Leftrightarrow \mathrm{H}_{2} \mathrm{O}+\mathrm{O}_{2}$ & $0.5000 \mathrm{e} 14$ & 0.00 & 1000 \\
\hline 10 & $\mathrm{H}+\mathrm{HO}_{2} \Leftrightarrow \mathrm{H}_{2}+\mathrm{O}_{2}$ & $0.2530 \mathrm{e} 14$ & 0.00 & 700 \\
\hline 11 & $\mathrm{H}+\mathrm{HO}_{2} \Leftrightarrow \mathrm{OH}+\mathrm{OH}$ & $0.1990 \mathrm{e} 13$ & 0.00 & 1800 \\
\hline 12 & $\mathrm{O}+\mathrm{HO}_{2} \Leftrightarrow \mathrm{O}_{2}+\mathrm{OH}$ & $0.5000 \mathrm{e} 12$ & 0.00 & 1000 \\
\hline 13 & $\mathrm{HO}_{2}+\mathrm{HO}_{2} \Leftrightarrow \mathrm{O}_{2}+\mathrm{H}_{2} \mathrm{O}_{2}$ & $0.1990 \mathrm{e} 13$ & 0.00 & 0 \\
\hline 14 & $\mathrm{H}_{2}+\mathrm{HO}_{2} \Leftrightarrow \mathrm{H}+\mathrm{H}_{2} \mathrm{O}_{2}$ & $0.3010 \mathrm{e} 12$ & 0.00 & 18700 \\
\hline 15 & $\mathrm{OH}+\mathrm{H}_{2} \mathrm{O}_{2} \Leftrightarrow \mathrm{HO}_{2}+\mathrm{H}_{2} \mathrm{O}$ & $0.1020 \mathrm{e} 14$ & 0.00 & 1900 \\
\hline 16 & $\mathrm{H}+\mathrm{H}_{2} \mathrm{O}_{2} \Leftrightarrow \mathrm{OH}+\mathrm{H}_{2} \mathrm{O}$ & $0.5000 \mathrm{e} 15$ & 0.00 & 10000 \\
\hline 17 & $\mathrm{O}+\mathrm{H}_{2} \mathrm{O}_{2} \Leftrightarrow \mathrm{OH}+\mathrm{HO}_{2}$ & $0.1990 \mathrm{e} 14$ & 0.00 & 5900 \\
\hline 18 & $\mathrm{H}_{2} \mathrm{O}_{2}+\mathrm{M} \Leftrightarrow \mathrm{OH}+\mathrm{OH}+\mathrm{M}$ & $0.1210 \mathrm{e} 18$ & 0.00 & 45500 \\
\hline
\end{tabular}


The chain-branching reactions in the $\mathrm{H}_{2} /$ air system are reactions 2,3 , and 4 in Table A1. Kuo (1986) indicates that reaction 2 is the rate-controlling step in the chainbranching explosion, as it is the most endothermic. Looking at reactions 2-4 as a standalone system wherein $\mathrm{H}_{2}$ and $\mathrm{O}_{2}$ are consumed and $\mathrm{H}_{2} \mathrm{O}$ and $\mathrm{H}$ radicals are produced, we see that for each $\mathrm{H}$ radical consumed, $3 \mathrm{H}$ radicals are produced, for a net of $2 \mathrm{H}$ radicals. Thus the net production rate of $\mathrm{H}$ radicals in the chain-branching system is 2 times the forward rate constant of reaction 2, or $2 \mathrm{k}_{\mathrm{f} 2}$, multiplied by the concentrations of $\mathrm{H}$ and $\mathrm{O}_{2}$. The balancing reaction that prevents the mixture of $\mathrm{H}_{2}$ and $\mathrm{O}_{2}$ from running away into an explosion is reaction 8 , wherein $\mathrm{HO}_{2}$ is produced. $\mathrm{HO}_{2}$ is relatively non-reactive, thus terminating the branching reaction chain. Since reaction 8 contains a $3^{\text {rd }}$ body, it is dependent on the number density of molecules, and thus on pressure as well as temperature. Therefore, for a given temperature, the importance of the $\mathrm{HO}_{2}$ producing chain terminating reaction is dependant upon the pressure. If we eliminate $\mathrm{HO}_{2}$ from the chemical mechanism, we must determine whether or not we are significantly influencing the ignition process. In order to determine this, we need to calculate the temperature at which the $\mathrm{H}$ radical consumption reaction just balances the $\mathrm{H}$ radical production system of reactions. If this temperature is significantly below the post-shock temperature, then we can safely ignore $\mathrm{HO}_{2}$, and consequently $\mathrm{H}_{2} \mathrm{O}_{2}$ that is produced from the $\mathrm{HO}_{2}$.

The equality that must be solved for the critical temperature is

$2 k_{f 2}[H][O 2]=k_{f 8}[H][O 2][M]$

where $[M]=\frac{p}{R_{u} T}$, as defined by Kuo (1986), the concentration of molecules available for collisions, i.e. molar density.

As mentioned previously, each reaction rate constant is of the form

$$
k_{f}=A * T^{n} * e^{\frac{-E a}{R_{u} T}}
$$

So we can substitute the appropriate values from Table A1 into equation (43) and simplify.

$$
\left(0.284 \times 10^{15}\right) * e^{\frac{-16400}{R_{u} T}}=\left(0.320 \times 10^{19}\right)\left(\frac{1}{T}\right)\left(\frac{p}{R_{u} T}\right)
$$

Solving for temperature at a pressure of 27 atmospheres, the pressure immediately behind the shock wave, we find that the creation and consumption of the $\mathrm{H}$ radical equilibrate at about $1300 \mathrm{~K}, 350$ degrees $\mathrm{K}$ below the expected post-shock temperature. Thus we can conclude that the $\mathrm{HO}_{2}$ reactions should not be a significant factor for this study. 


\section{References}

Anderson, Jr., J.D. Computational Fluid Dynamics, McGraw-Hill, Inc., New York, 1995.

Cambier, J.-L.; Adelman, H.G. "Preliminary Numerical Simulations of a Pulsed Detonation Wave Engine," AIAA Paper 88-2960, July 1988.

Carpenter, M.H. “Three-Dimensional Computations of Cross-Flow Injection and Combustion in a Supersonic Flow," AIAA 89-1870, June 1989.

Coleman, M.L. "Overview of Pulse Detonation Propulsion Technology," CPIA Report No. CPTR 70, April, 2001.

Drummond J.P.; Mukunda, H.S. “A Numerical Study of Mixing Enhancement in Supersonic Reacting Flow Fields,” AIAA Paper 88-3260, July 1988.

Drummond, J.P. "A Two-Dimensional Numerical Simulation of a Supersonic, Chemically Reacting Mixing Layer," NASA TM-4055, 1988.

Drummond, J.P. “Supersonic Reacting Internal Flow Fields,” NASA TM-103480, 1990.

Drummond, J.P.; Carpenter, M.H.; White, J.A. "Development and Application of the SPARK Combustion Codes," $10^{\text {th }}$ National Aero-Space Plane Technology Symposium, April 1991.

Drummond, J.P. "Supersonic Reacting Internal Flowfields," in Numerical Approaches to Combustion Modeling, E.S. Oran and J.P. Boris, Eds., AIAA, Washington, D.C., 1991.

Gordon S.; McBride, B.J. “Computer Program for Calculation of Complex Chemical Equilibrium Compositions and Applications," NASA Reference Publication 1311, 1994.

Jachimowski, C.J. "An Analytical Study of the Hydrogen-Air Reaction Mechanism with Application to Scramjet Combustion," NASA Technical Paper 2791, February 1988.

Kaemming, T.A. "Integrated Vehicle Comparison of Turbo-Ramjet Engine and Pulsed Detonation Engine (PDE),” ASME Paper 2001-GT-0451, June 2001.

Kailasanath, K. “Applications of Detonations to Propulsion: A Review," AIAA Paper 99-1067, January, 1999.

Kailasanath, K.; Patnaik, G. "Pulsed Detonation Engines - What is its Performance," Proceedings, JANNAF Combustion Meeting, November 1999. 
Kailasanath, K.; Patnaik, G.; Li, C. “On Factors Controlling the Performance of Pulsed Detonation Engines", in Control of Detonation Processes, G. Roy, et al, editors. ElexKM, Moscow, 2000.

Kee, R.J.; Rupley, F.M.; Miller, J.A. “Chemkin-II: A FORTRAN Chemical Kinetics Package for the Analysis of Gas-Phase Chemical Kinetics," Sandia National Laboratories Report SAND89-8009, 1989.

Kuo, K.K. Principles of Combustion, John Wiley \& Sons, New York, 1986.

Lynch, E.D.; Edelman, R. "Computational Fluid Dynamic Analysis of the Pulse Detonation Engine Concept,” AIAA Paper 94-0264, January 1994.

Nichols, J.; Wilkinson, H.; Morrison, R. "Intermittent Detonation as a Thrust Producing Mechanism," Jet Propulsion Journal of the American Rocket Society 27 [5] (1957), 534-541.

Oates, G.C. Aerothermodynamics of Gas Turbine and Rocket Propulsion, AIAA, Washington D.C., 1984.

Poinsot, T.J.; Lele, S.K. "Boundary Conditions for Direct Simulations of Compressible Viscous Flows,” Journal of Computational Physics, 101, (1992), 104-129.

Radhakrishnan, K. "Combustion Kinetics and Sensitivity Analysis," in Numerical Approaches to Combustion Modeling, E.S. Oran and J.P. Boris, Eds., AIAA, Washington D.C., 1991.

Shapiro, A.H. The Dynamics and Thermodynamics of Compressible Fluid Flow, Ronald Press Company, New York, 1953.

Shepherd, J.E. "Chemical Kinetics of Hydrogen-Air-Diluent Detonation," in Progress in Astronautics and Aeronautics, Vol. 106, p. 263-293, AIAA, New York, 1986.

Smith R.E.; Weigel, B.L. "Analytic and Approximate Boundary-Fitted Coordinate Systems for Fluid Flow Simulation,” AIAA Paper 80-0192, January 1980.

Sterling, J.; Ghorbanian, K.; Humphrey, J.; Sobota, T. "Numerical Investigations of Pulse Detonation Wave Engines,” AIAA Paper 95-2479, July 1995.

Thaker A.A.; Chelliah, H.K. "Numerical Prediction of Oblique Detonation Wave Structures Using Detailed and Reduced Reaction Mechanisms," Combustion Theory Modelling, [1], (1997) 347-376. 
Williams, F.A. Combustion Theory, Addison-Wesley Publishing Company, Inc., Menlo Park, 1985.

Wilson, J.; Paxson, D.E. "On the Exit Boundary Condition for One-dimensional Calculations of Pulsed Detonation Engine Performance," Proceedings, International Colloquium on Detonation, Explosion, and Reactive Systems, July 2001. 


\begin{tabular}{|c|c|c|c|}
\hline \multicolumn{3}{|c|}{ REPORT DOCUMENTATION PAGE } & $\begin{array}{l}\text { Form Approved } \\
\text { OMB No. 0704-0188 }\end{array}$ \\
\hline \multicolumn{4}{|c|}{ 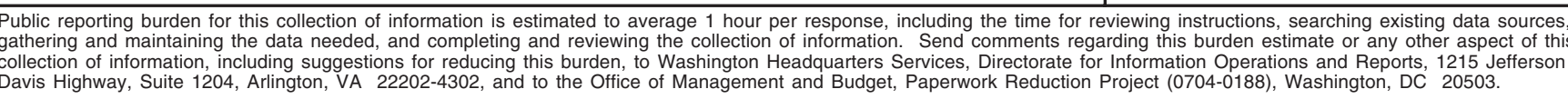 } \\
\hline 1. AGENCY USE ONLY (Leave blank) & $\begin{array}{l}\text { 2. REPORT DATE } \\
\text { August } 2002\end{array}$ & $3 . \mathrm{F}$ & $\begin{array}{l}\text { ID DATES COVERED } \\
\text { echnical Memorandum }\end{array}$ \\
\hline \multicolumn{4}{|c|}{ Effects of Fuel Distribution on Detonation Tube Performance } \\
\hline $\begin{array}{l}\text { 6. AUTHOR(S) } \\
\text { Hugh Douglas Perkins }\end{array}$ & & & WU-708-48-13-00 \\
\hline \multicolumn{3}{|c|}{$\begin{array}{l}\text { 7. PERFORMING ORGANIZATION NAME(S) AND ADDRESS(ES) } \\
\text { National Aeronautics and Space Administration } \\
\text { John H. Glenn Research Center at Lewis Field } \\
\text { Cleveland, Ohio } 44135-3191\end{array}$} & $\begin{array}{l}\text { 8. PERFORMING ORGANIZATION } \\
\text { REPORT NUMBER } \\
\text { E-13463 }\end{array}$ \\
\hline \multicolumn{3}{|c|}{$\begin{array}{l}\text { 9. SPONSORING/MONITORING AGENCY NAME(S) AND ADDRESS(ES) } \\
\text { National Aeronautics and Space Administration } \\
\text { Washington, DC 20546-0001 }\end{array}$} & $\begin{array}{l}\text { 10. SPONSORING/MONITORING } \\
\text { AGENCY REPORT NUMBER } \\
\text { NASA TM-2002-211712 }\end{array}$ \\
\hline
\end{tabular}

Responsible person, Hugh Douglas Perkins, organization code 0142, 216-977-7414.

12a. DISTRIBUTION/AVAILABILITY STATEMENT

12b. DISTRIBUTION CODE

Unclassified - Unlimited

Subject Category: 07

Distribution: Nonstandard

Available electronically at http://gltrs.grc.nasa.gov/GLTRS

This publication is available from the NASA Center for AeroSpace Information, 301-621-0390.

13. ABSTRACT (Maximum 200 words)

A pulse detonation engine (PDE) uses a series of high frequency intermittent detonation tubes to generate thrust. The process of filling the detonation tube with fuel and air for each cycle may yield non-uniform mixtures. Lack of mixture uniformity is commonly ignored when calculating detonation tube thrust performance. In this study, detonation cycles featuring idealized non-uniform $\mathrm{H}_{2}$ /air mixtures were analyzed using the SPARK two-dimensional Navier-Stokes CFD code with 7-step $\mathrm{H}_{2}$ /air reaction mechanism. Mixture non-uniformities examined included axial equivalence ratio gradients, transverse equivalence ratio gradients, and partially fueled tubes. Three different average test section equivalence ratios $(\Phi)$, stoichiometric $(\Phi=1.00)$ fuel lean $(\Phi=0.90)$ and fuel rich $(\Phi=1.10)$ were studied. All mixtures were detonable throughout the detonation tube. It was found that various mixtures representing the same test section equivalence ratio had specific impulses within 1 percent of each other, indicating that good fuel/air mixing is not a prerequisite for optimal detonation tube performance.

\begin{tabular}{|c|c|c|}
\hline \multicolumn{3}{|l|}{$\begin{array}{l}\text { 14. SUBJECT TERMS } \\
\text { Pulse detonatior } \\
\text { fluid dynamics }\end{array}$} \\
\hline $\begin{array}{l}\text { 17. SECURITY CLASSIFICATION } \\
\text { OF REPORT }\end{array}$ & $\begin{array}{l}\text { 18. SECURITY CLASSIFICATION } \\
\text { OF THIS PAGE }\end{array}$ & $\begin{array}{l}\text { 19. SECURITY CLASSIFICATION } \\
\text { OF ABSTRACT }\end{array}$ \\
\hline Unclassified & Unclassified & Unclassified \\
\hline
\end{tabular}

NSN 7540-01-280-5500

Standard Form 298 (Rev. 2-89) Prescribed by ANSI Std. Z39-18
298-102 